\title{
Attenuating Magnetic Interference in a UAV System
}

\author{
by \\ Matthew Wells
}

\author{
A Thesis submitted to \\ the Faculty of Graduate Studies and Researcl \\ in partial fulfilment of \\ the requirements for the degree of \\ Master of Applied Science
}

Ottawa-Carleton Institute for

Mechanical and Aerospace Engineering

Department of Mechanical and Aerospace Engineering

Carleton University

Ottana. Ontario. Canada

2008

Copyright (C)

2008 - Matthew Wells 


$\begin{array}{ll}\begin{array}{l}\text { Library and } \\ \text { Archives Canada }\end{array} & \begin{array}{l}\text { Bibliothèque et } \\ \text { Archives Canada }\end{array} \\ \begin{array}{l}\text { Published Heritage } \\ \text { Branch }\end{array} & \begin{array}{l}\text { Direction du } \\ \text { Patrimoine de l'édition }\end{array} \\ \begin{array}{l}\text { 395 Wellington Street } \\ \text { Ottawa ON K1A 0N4 } \\ \text { Canada }\end{array} & \begin{array}{l}\text { 395, rue Wellington } \\ \text { Ottawa ON K1A 0N4 } \\ \text { Canada }\end{array}\end{array}$

Your file Votre référence ISBN: 978-0-494-44061-2 Our file Notre référence ISBN: 978-0-494-44061-2

NOTICE:

The author has granted a nonexclusive license allowing Library and Archives Canada to reproduce, publish, archive, preserve, conserve, communicate to the public by telecommunication or on the Internet, loan, distribute and sell theses worldwide, for commercial or noncommercial purposes, in microform, paper, electronic and/or any other formats.

The author retains copyright ownership and moral rights in this thesis. Neither the thesis nor substantial extracts from it may be printed or otherwise reproduced without the author's permission.
AVIS:

L'auteur a accordé une licence non exclusive permettant à la Bibliothèque et Archives Canada de reproduire, publier, archiver, sauvegarder, conserver, transmettre au public par télécommunication ou par l'Internet, prêter, distribuer et vendre des thèses partout dans le monde, à des fins commerciales ou autres, sur support microforme, papier, électronique et/ou autres formats.

L'auteur conserve la propriété du droit d'auteur et des droits moraux qui protège cette thèse. $\mathrm{Ni}$ la thèse ni des extraits substantiels de celle-ci ne doivent être imprimés ou autrement reproduits sans son autorisation.
In compliance with the Canadian Privacy Act some supporting forms may have been removed from this thesis.

While these forms may be included in the document page count, their removal does not represent any loss of content from the thesis.
Conformément à la loi canadienne sur la protection de la vie privée, quelques formulaires secondaires ont été enlevés de cette thèse.

Bien que ces formulaires aient inclus dans la pagination, il n'y aura aucun contenu manquant.

\section{Canada}




\section{Abstract}

This thesis presents the development of a novel strategy for minimizing magnetic interference in a UAV system through design optimization of the position and orientation of magnetic field sources. The method, called Optimal Field Attenuation (OFA) was developed for the GeoSurv II ummanned aircraft being designed at Carleton University to perform autonomous geomagnetic surveys; to ensure high precision survey data it is necessary to eliminate the magnetic interference produced by the aircraft. Two dipole equivalence modelling (DEM) methods, Direct Dipole Modelling (DEM) and Modified Dipole Ellipse Modelling (MoDEM) were proposed. The MoDEM algorithm was developed uniquely for this thesis; it was experimentally validated and proven superior to DEM for use with OFA.

The three objectives of OFA are to attenuate, to minimize the field variation over the attenuation area, and when multiple attenuation areas are defined, to equalize the magnetic field (at each area) produced by a system of magnetic sources. OFA was experimentally validated and it was determined that field attenuation and equalization decrease, and spatial stability increases with respect to the size of the optimization area. It was also determined that increased degrees of freedom (the number of configurable sources of interference) results in improved OFA performance. 


\section{Acknowledgments}

Completing this thesis would not have been possible without a great deal of help. I would like to thank my supervisors, Mojtaba Ahmadi and Paul Straznicky, for their guidance, patience, and advice in overcoming the technical challenges experienced during the course of my research. I would like to thank my partner, Isabel, for countless hours spent proofreading. Finally I would like to thank former students on the GeoSurv II project, Ahmed Soliman, Emad Zammar, Sheilagh O'Leary, and Radman Rabii, for their help completing magnetic signature testing. 


\section{Table of Contents}

Abstract $\quad$ iii

Acknowledgments $\quad$ iv

Table of Contents $\quad$ v

List of Tables $\quad$ ix

List of Figures $\quad$ x

Nomenclature xiii

1 Introduction 1

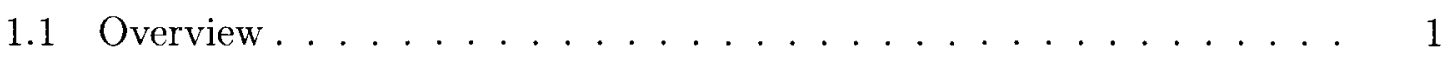

1.2 Research Motivation .................. 2

1.2 .1 GeoSurv II UAV . . . . . . . . . . . . . . 2

1.2.2 Research Objectives ................ 6

1.2.3 Research Contributions .............. 7

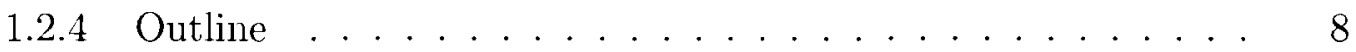

2 Theory 11

2.1 Magnetic Fields . . . . . . . . . . . . . . . 11

2.1.1 Field Strength vs. Magnetic Flux Density/Magnetic Induction 11 
2.1 .2 Magnetic Interference . . . . . . . . . . . . . . . 12

2.2 Modelling Magnetic Fields . . . . . . . . . . . . . . . . 14

2.2 .1 The Biot-Savart Law . . . . . . . . . . . . . . . . . 14

2.2 .2 Magnetic Dipole . . . . . . . . . . . . . . . . 15

2.2 .3 Dipole Equivalence . . . . . . . . . . . . . . . . . 17

2.2.4 Survey of Equivalent Dipole Modelling Strategies . . . . . . 19

2.3 Geomagnetism . . . . . . . . . . . . . . . 21

2.3 .1 The Earth's Magnetic Field . . . . . . . . . . . . . 21

3 Low Magnetic Signature Actuation System 23

3.1 Introduction . . . . . . . . . . . . . . . . . 23

3.2 Low Magnetic Actuators for GeoSurv II . . . . . . . . . . 23

3.3 Survey of Alternative Actuation Schemes . . . . . . . . . . . . 24

3.3.1 Fluid Power Actuation . . . . . . . . . . . . . 24

3.3 .2 Solid State Actuators . . . . . . . . . . . . . 25

3.3 .3 Hybrid Actuators . . . . . . . . . . . . . 26

$3.3 .4 \quad$ Flapless Control . . . . . . . . . . . . . . . . . . . . 29

3.3 .5 Actuator Selection . . . . . . . . . . . . . 30

3.4 Strategies for Minimizing Magnetic Fields . . . . . . . . . . 30

3.4 .1 Passive Techniques . . . . . . . . . . . . . . . . . 31

3.4 .2 Active Techniques. . . . . . . . . . . . . . . 32

3.5 Optimal Field Attenuation $(\mathrm{OFA}) \ldots \ldots \ldots \ldots \ldots$

4 Measuring Magnetic Fields $\quad 35$

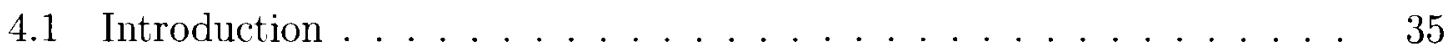

4.2 Magnetic Anomaly Detection (MAD) Shack Facility . . . . . . . . 35

4.2.1 Optically Pumped Cesium Magnetometer . . . . . . . . . . 36

4.2.2 Fluxgate (Saturable-Core) Magnetometer . . . . . . . . . . 36 
4.3 GeoSurv II Magnetic Measurement System . . . . . . . . . . . . 37

4.3.1 Hardware Selection . . . . . . . . . . . . . . . 37

4.3.2 Data Acquisition System . . . . . . . . . . . . 38

4.3.3 System Configuration . . . . . . . . . . . . 38

4.3.4 Measurement Algorithm . . . . . . . . . . . . 40

4.3.5 Measurement System Analysis . . . . . . . . . . . . . . . 41

5 Magnetic Field Modelling $\quad 44$

5.1 Introduction . . . . . . . . . . . . . . . . 44

5.2 Data Acquisition . . . . . . . . . . . . . . . 44

5.2.1 Test Objects..................... 45

5.3 Dipole Modelling . . . . . . . . . . . . . . . . 47

5.3.1 Direct Dipole Parameterization ... . . . . . . . . . 49

5.3.2 Direct Dipole Test Results . . . . . . . . . . . . . 49

5.4 MoDEM Modelling . . . . . . . . . . . . . . . . 53

5.4 .1 MoDEM Model Formulation . . . . . . . . . . . . 53

5.4.2 MoDEM Parameterization .............. 55

5.4 .3 MoDEM Test Results . . . . . . . . . . . . 57

5.5 Model Selection ................... 60

6 Optimal Field Attenuation $\quad 66$

6.1 Introduction . . . . . . . . . . . . . . . 66

6.2 OFA Background ..................... 67

6.2 .1 Time Variant vs. Time Invariant . . . . . . . . . . . 68

6.2.2 Optimal Attenuation ................ 68

6.2 .3 Spatial Field Stability . . . . . . . . . . . . 68

6.2.4 Field Equalization ................. 70

6.3 Optimal Field Attenuation Algorithm . . . . . . . . . . . . 70 


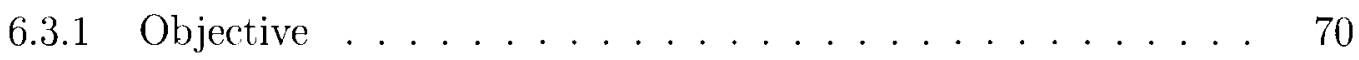

6.3 .2 Constraints . . . . . . . . . . . . . 72

6.4 OFA Verification Experiment $\ldots \ldots \ldots \ldots \ldots \ldots \ldots$

6.4 .1 Test Configuration . . . . . . . . . . . 73

6.4 .2 Results . . . . . . . . . . . . . . . . . 74

6.4 .3 Analysis . . . . . . . . . . . . . . . . 75

6.5 OFA Analysis $\ldots \ldots \ldots \ldots \ldots \ldots \ldots \ldots \ldots$

6.5.1 Attenuation vs. Spatial Field Stability _. . . . . . . . 80

6.5.2 GeoSurv II Multi-Objective Field Equalization . . . . . . . 86

6.6 OFA for Use on GeoSurv II . . . . . . . . . . . . . . . . 90

$\begin{array}{lll}7 & \text { Conclusions and Recommendations } & 91\end{array}$

7.1 Summary . . . . . . . . . . . . . . . . . . . 9 91

7.2 Research Conclusions . . . . . . . . . . . . . . . . 92

7.2.1 Attenuation Strategies for GeoSurv II . . . . . . . . . . . 92

7.2.2 Low Magnetic Signature Actuators . . . . . . . . . . . . 93

7.2.3 Magnetic Field Modelling . . . . . . . . . . . . . . 93

7.2.4 Optimal Field Attenuation . . . . . . . . . . . . . . 94

7.3 Recommendations for Future Work . . . . . . . . . . . 95

$\begin{array}{ll}\text { List of References } & 97\end{array}$

$\begin{array}{lll}\text { Appendix A Actuator Selection Matrix } & 101\end{array}$

$\begin{array}{lll}\text { Appendix B Data Acquisition Scripts } & 102\end{array}$

$\begin{array}{lll}\text { Appendix C DDM and MoDEM Parameterization Scripts } & 107\end{array}$

$\begin{array}{ll}\text { Appendix D OFA Scripts } & 111\end{array}$ 


\section{List of Tables}

5.1 Mean Absolute Modelling Error (nT) for the DDM/P Field Estimation

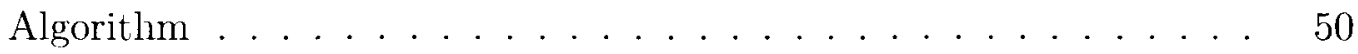

5.2 Mean Absolute Modelling Error (nT) for the MoDEM Field Estimation Algorithm . . . . . . . . . . . . . . . . 58

5.3 MoDEM improvement over DDP $(\mathrm{n} T) \ldots \ldots . \ldots 60$

6.1 OFA Verification Testing (all values in $\mathrm{nT}$ ) . . . . . . . 76

6.2 Theoretical vs. Experimental Attenuation (all values in $\mathrm{nT}$ ) . . . 76

6.3 UAV Planar Boundary Constraints for Figure 6.16 . . . . . . . . . 86

6.4 Left - Right Estimated Field Magnitude Differential (all values in nT) 89 


\section{List of Figures}

1.1 GeoSurv II UAV . . . . . . . . . . . . . . . . . . . . . 2

1.2 GeoSurv II Actuating System . . . . . . . . . . . . . . 4

2.1 Biot-Savart Law. . . . . . . . . . . . . . . . . . . 15

2.2 Simulated Point-Dipole Magnetic Field. . . . . . . . . . . 18

2.3 Normalized $\vec{B}$ vs. Rotation for a Point-Dipole. . . . . . . . . . 18

3.1 Miniature Trailing Edge Effectors. . . . . . . . . . . 25

3.2 Inchworm Operation. . . . . . . . . . . . . . . 27

3.3 Post-Buckled Precompressed (PBP) Control Surface. . . . . 28

3.4 Flapless Airfoil Illustration. . . . . . . . . . . . . . . . . . . . . 29

4.1 HMR-2300 Sensor. . . . . . . . . . . . . . . . . . 38

4.2 MAD Shack Facility schematic. . . . . . . . . . . . . . 39

4.3 Standard Deviation of $\vec{B}_{\text {environment }}$ vs. Sampling Hold Time . . 42

5.1 Dipole Parameterization Test Schematic . . . . . . . . . 45

5.2 Coordinate System Assignment - 2 and 3DP Field Sources . 46

5.3 Coordinate System Assignment - Hitec HSR-5995TG . . . . 46

5.4 Coordinate System Assignment - Futaba FP-S148 . . . . . 47

$5.5 \vec{B}$ vs. Applied Rotation: 2DP Direct Dipole Model . . . . . . 51

$5.6 \vec{B}$ vs. Applied Rotation: 3DPb Direct Dipole Model . . . . . . 51

$5.7 \vec{B}$ vs. Applied Rotation: Futaba FP-S148 Direct Dipole Model. 52

$5.8 \vec{B}$ vs. Applied Rotation: Hitec HSR-5995TG Direct Dipole Model 52 


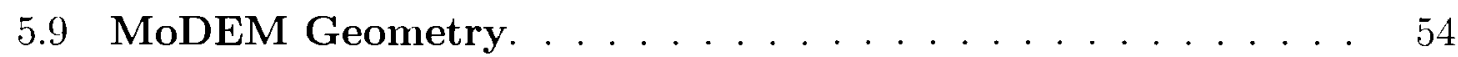

$5.10 \vec{B}$ vs. Applied Rotation: 2DP MoDEM . . . . . . . . 58

$5.11 \vec{B}$ vs. Applied Rotation: 3DPb MoDEM . . . . . . . . . 59

$5.12 \vec{B}$ vs. Applied Rotation: Futaba FP-S148 MoDEM . . . . . 59

$5.13 \vec{B}$ vs. Applied Rotation: Hitec HSR-5995TG MoDEM . . . . 60

5.14 Comparison Between MoDEM and Direct Dipole Model Es-

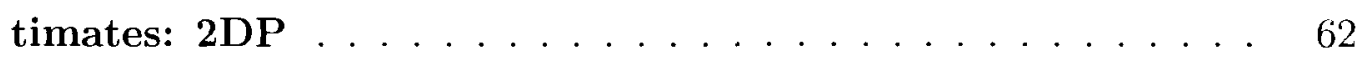

5.15 Comparison Between MoDEM and Direct Dipole Model Estimates: $3 \mathrm{DPb} \ldots \ldots \ldots \ldots \ldots \ldots \ldots \ldots$

5.16 Comparison Between MoDEM and Direct Dipole Model Estimates: Futaba FP-S148 . . . . . . . . . . . . . . . 63

5.17 Comparison Between MoDEM and Direct Dipole Model Estimates: Hitec HSR-5995TG . . . . . . . . . . . . 64

5.18 MoDEM Modelling Error - 3DP Source . . . . . . . . . 64

5.19 MoDEM Modelling Error - Hitec HSR-5995TG . . . . . . 65

6.1 HMR-2300 Circuit Schematic. . . . . . . . . . . . . . . 69

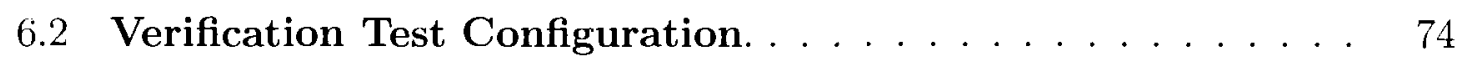

6.3 Initial Field Measure vs. Model $-\mathbf{1} \mathbf{c m} \times 1 \mathbf{c m}$ Area $\ldots \ldots .77$

6.4 Initial Field Measure vs. Model $-\mathbf{5} \mathbf{c m} \times \mathbf{5} \mathbf{c m}$ Area $\ldots \ldots .77$

6.5 Optimized Field Measure vs. Model $-\mathbf{1} \mathbf{c m} \times \mathbf{1} \mathbf{c m}$ Area . . . 78

6.6 Optimized Field Measure vs. Model $-\mathbf{5} \mathbf{c m} \times \mathbf{5} \mathbf{c m}$ Area . . . 78

6.7 Field Attenuation Measure vs. Model $-\mathbf{1} \mathbf{c m ~ x ~} \mathbf{1} \mathbf{c m}$ Area . . . 79

6.8 Field Attenuation Measure vs. Model - $\mathbf{5} \mathbf{c m}$ x $\mathbf{5} \mathbf{c m}$ Area . . . 79

6.9 Attenuation vs. Spatial Field Stability Test Configuration. . 80

6.10 Average (attenuated) Field Intensity vs. Area of Optimization 82

6.11 Percent Attenuation vs. Area of Optimization . . . . . . . 82

6.12 Stability Metric vs. Area of Optimization . . . . . . . 83 
6.13 Initial Field Distribution - Full Flexibility Case . . . . . . 84

$6.141 \mathrm{~mm} \times 1 \mathrm{~mm}$ Optimization Area Field Distribution - Full Flexibility Case $\ldots \ldots \ldots \ldots \ldots \ldots \ldots \ldots$

$6.155 \mathrm{~cm}$ x 5cm Optimization Area Field Distribution - Full Flexibility Case $\ldots \ldots \ldots \ldots \ldots \ldots \ldots$

6.16 Constraint Regions for the GeoSurv II Primary Flight Control Actuators. . . . . . . . . . . . . . . . . 87

6.17 Post-Optimized Left-Right Field Differential _ . . . . . . . 89 


\section{Nomenclature}

List of Symbols: The following symbols are used throughout the thesis:

\begin{tabular}{|c|c|c|}
\hline Symbol & Description & Units \\
\hline$\vec{B}$ & Magnetic Flux Density & nanoTesla (nT) \\
$\|\vec{B}\|$ & Magnetic Field Intensity & nanoTesla $(\mathrm{nT})$ \\
$\vec{H}$ & Magnetic Field Strength & amperes/meter $(\mathrm{A} / \mathrm{m})$ \\
$\vec{m}$ & Magnetic Dipole Moment & amperes*meter ${ }^{2}\left(\mathrm{Am}{ }^{2}\right)$ \\
$\vec{R}$ & Observation Position & meters $(\mathrm{m})$ \\
$\mu_{o}$ & Magnetic Permeability (vacuum) & Henry's/meter $(\mathrm{H} / \mathrm{m})$ \\
$\mu_{r}$ & Relative Magnetic Permeability & $\frac{H / m}{H / m}$ \\
$I$ & Current & amperes $(\mathrm{A})$ \\
$\vec{r}$ & Position Vector & meters $(\mathrm{m})$ \\
$\hat{r}$ & Unit Position Vector & $(\mathrm{m} / \mathrm{m})$ \\
$d l$ & Differential Length & meters $(\mathrm{m})$ \\
\hline
\end{tabular}


List of Acronyms: The following acronyms are used throughout the thesis:

\begin{tabular}{|c|c|}
\hline Acronym & Description \\
\hline OFA & Optimal Field Attenuation \\
DEM & Dipole Equivalence Model \\
DDM & Direct Dipole Model \\
DDP & Direct Dipole Parameterization \\
MoDEM & Modified Dipole Ellipse Model \\
DAQ & Data Acquisition \\
MAD & Magnetic Anomoly Detection \\
UAV & Unmanned Air Vehicle \\
SDM & Smart Digital Magnetometer \\
VDC & Volts Direct Current \\
2DP & 2 Magnet Dipole \\
3DP & 3 Magnet Dipole \\
\hline
\end{tabular}




\section{Chapter 1}

\section{Introduction}

\subsection{Overview}

Many modern electronic systems are protected from the effects of magnetic interference. The majority of these systems apply magnetic shielding strategies in which sensitive components are physically separated by a material which acts as a barrier to a magnetic field. Systems using magnetic field sensors (magnetometers) are uniquely susceptible to the effects of magnetic interference.

For airborne geomagnetic surveys, magnetometers are installed in an aircraft and used to measure the magnetic field over a geological region of interest. Magnetic field data must be measured at a very high resolution (less than 0.01 nanoTesla $(\mathrm{nT})$ ) to indicate the presence of oil or minearal deposits, for example. Magnetometers measure the total magnetic field, which includes the environmental background field, superimposed on contributions from magnetic materials and extremely low frequency $(<10 \mathrm{~Hz})$ electromagnetic sources on the aircraft. The task of attenuating magnetic interference is particularly challenging in geomagnetic surveying; it is not so much a question of reducing signal noise as it is minimizing the extraneous field components such that only the local envirommental magnetic field is measured. 
Traditional electromagnetic shielding solutions are not suitable for the protection of magnetometer-based devices because they either add a barrier to the magnetic fields that the sensor was designed to measure, or add additional magnetic interference by altering the local magnetic field in the vicinity of the sensor. This thesis presents the development and experimental verification of a novel strategy for attelluating magnetic interference in an unmanned geo-surveying aircraft.

\subsection{Research Motivation}

\subsubsection{GeoSurv II UAV}

GeoSurv II (Figure 1.1) is an Unmanned Air Vehicle (UAV) being developed at Carleton University. The vehicle is a medium sized UAV with a wingspan of 4.88 $\mathrm{m}$, length of $4.36 \mathrm{~m}$ (nose to tail), and a target mass of $90.7 \mathrm{~kg}$ (excluding fuel). The design team is composed of faculty members, industry professionals, graduate students, and a revolving group (year to year) of fourth year aerospace, mechanical, and computer systems engineering students.

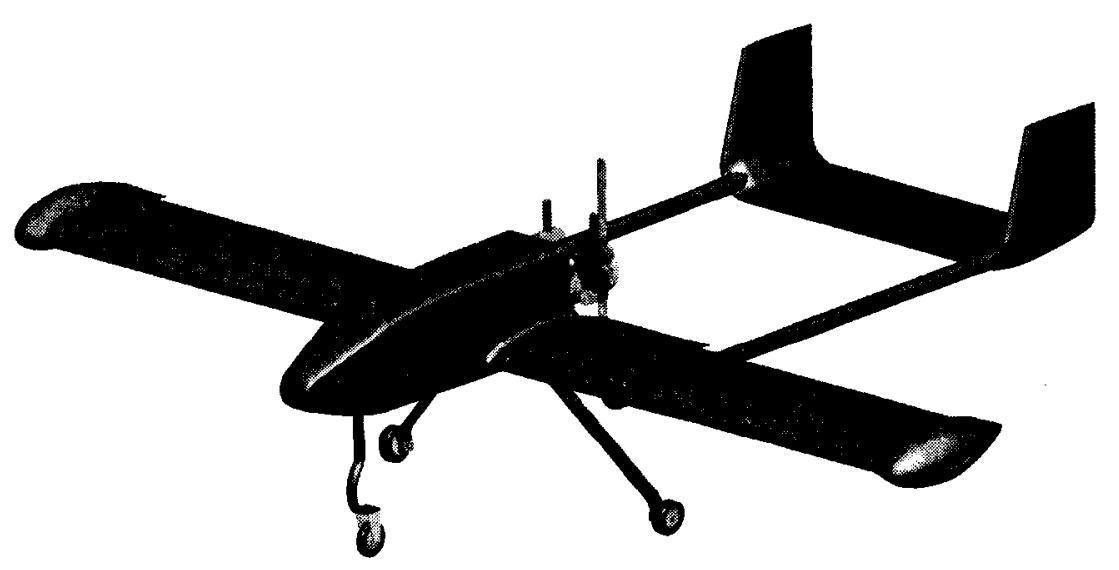

Figure 1.1: GeoSurv II UAV 
The primary mission driving the design of the aircraft is unmanned, autonomous geomagnetic surveys with an emphasis on low altitude, high resolution data acquisition over smaller survey areas than manned aircraft currently perform. GeoSurv II will be equipped with multiple high resolution magnetometers which are sensitive to magnetic interference generated by the vehicle itself. Magnetic interference may arise as a result of magnetic materials, electrical components, or electric currents induced in conductive materials on-board the aircraft [1].

The goal of developing a system which is capable of delivering higher resolution data than is currently feasible using nimned aircraft requires that the uav lave magnetic interference eliminated to the greatest extent possible. The flight control actuation system represents a class of components and subsystems that are normally not designed with the aim of minimizing the magnetic signature. Therefore, an important area of research and development on the UAV is minimizing the magnetic signature of the the actuating system.

\section{GeoSurv II Actuating System}

The actuating system on GeoSurv II consists of primary flight control systems and secondary aircraft control systems. Primary components refer to the aileron, rudder, and elevator actuators, control surfaces, and associated infrastructure (control rods, power cables, etc). Secondary systems refer to the engine throttle control actuator, generator, and landing (parafoil) control actuators and associated infrastructure. Figure 1.2 highlights and identifies the locations of actuating components on GeoSurv II.

The principal focus of this thesis will be the primary actuation system components. The aileron system, in particular, will be located in close proximity to the wingtip 


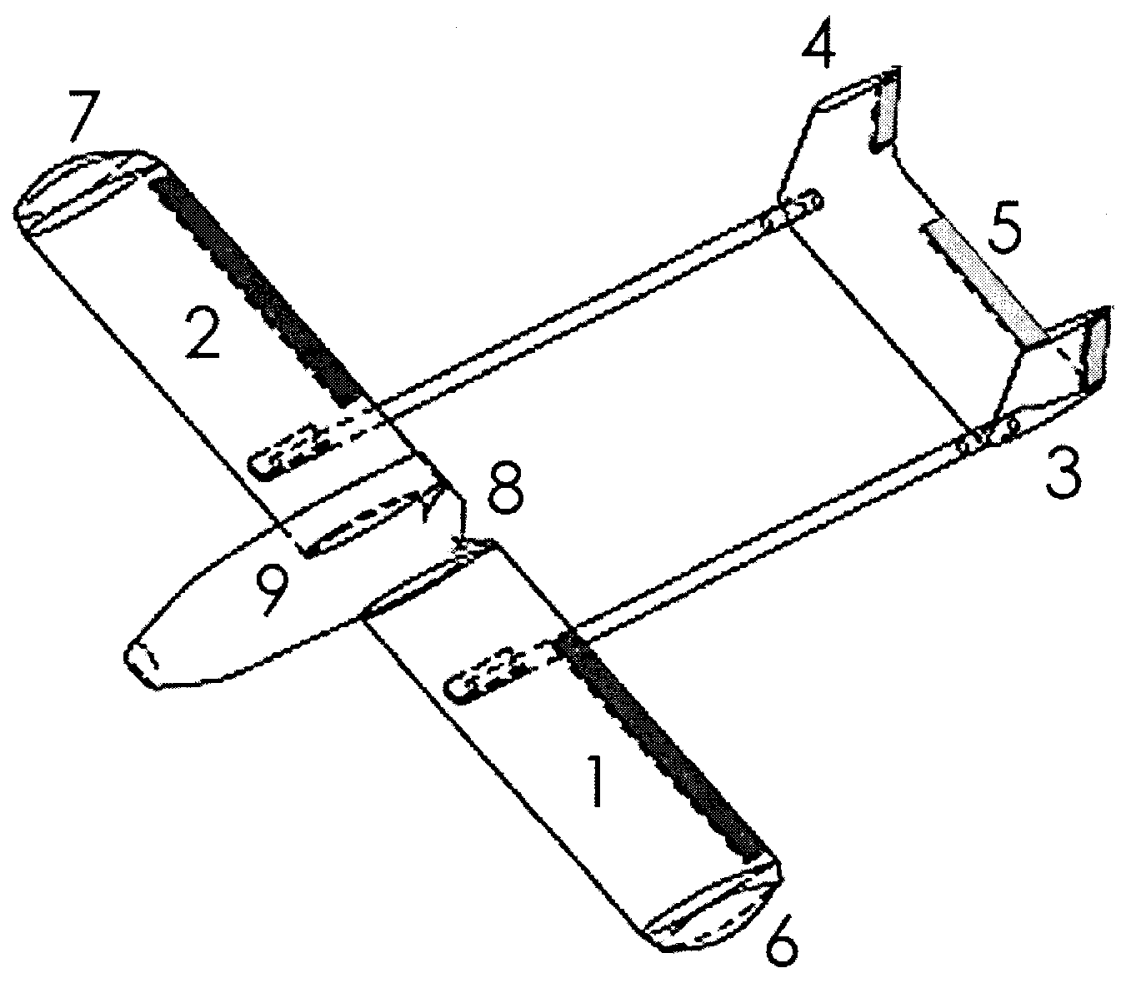

Figure 1.2: GeoSurv II Actuating System 1.) Left Aileron; 2.) Riglit Aileron; 3.) Left Rudder; 4.) Right Rudder; 5.) Elevator; 6.) Left Magnetometer; 7.) Right Magnetometer; 8.) Engine/Generator; 9.) Throttle and Parafoil Recovery System

magnetometers; furthermore the control surfaces will undergo significant motions with respect to the magnetometers. These factors make the flight control actuating system the primary area of concern in terms of magnetic interference. Secondary components, such as the recovery system, engine, and generator will also be considered, however on a secondary basis. The recovery system includes a parafoil and actuators for steering the aircraft during an unpowered descent. It will not be operational during a survey mission; furthermore the system has not been designed in detail at this time making it difficult to analyze, predict, or develop minimization strategies for the interference it will generate. 


\section{Magnetic Signature Requirements}

Specific design requirements were developed with input from Sander Geophysics Ltd. (SGL), an Ottawa-based geological exploration company specializing in airborne magnetic, gravimetric, and radiometric surveys. SGL currently operates a fleet of manned aircraft which are deployed on missions worldwide. Unmanned vehicles were identified as a possible tool for conducting higher resolution magnetic surveys (tighter survey grid and lower altitude) than is possible with manned vehicles. In addition to performance, deployability, and reliability requirements, GeoSurv II should exceed the performance of manned survey vehicles in terms of the level of magnetic interference introduced into the system. Requirements pertaining to low magnetic signature of the vehicle include [1]:

- Requirement 3.1.1-3 Uncompensatable magnetic fields (noise) generated by the UAV shall not exceed 0.1 nano Tesla $(n T)+/-0.05 n T$ for fixed sources.

- Requirement 3.1.1-3 Uncompensatable magnetic fields (noise) generated by the UAV shall not exceed $0.01 n T+/-0.005 n T$ for time-varying sources, in a 0 to $5 \mathrm{~Hz}$ bandwidth.

- Requirement 3.1.1-9 The UAV structure shall consist of only composite materials within $30 \mathrm{~cm}$ of the magnetometer sensors. Use of metals within this area shall be minimized and shall in any case be limited to non-magnetic hardware components.

- Requirement 3.1.3-4 If 2 magnetometers are carried, these shall be mounted in horizontal gradiometer configuration.

- Requirement 3.1.3-5 The UAV shall carry 1 or 2 G822A magnetometer sensors. 
- Requirement 3.1.3-6 The UAV shall carry 1 Billingsley TFM 100 vector magnetometer sensor.

- Requirement 3.1.3-7 All materials within 1 foot (30 centimeters) of magnetometer(s) shall be non-conductive, with the exception of small non-magnetic fasteners.

The magnetometers currently used by SGL have a measurement resolution below $0.01 \mathrm{nT}$; the requirements seek to maximize the achievable resolution by eliminating sources of interference. Although the Eartlı's field varies, in the Ottawa area the magnitude is on the order of 55,000 nT. A design goal of limiting interference to $0.01 \mathrm{nT}$ is most significant for the case of magnetic gradient surveys. Gradiometry refers to measuring the change in magnetic field over a fixed distance; in the case of GeoSurv II this would be across the wingspan. The gradient measurement is indicative of sudden changes in the magnetic field which may refer to the boundaries of geological items of interest. Because the magnetic gradient is a differential measure across a relatively small distance compared to the size of a survey area the value may very well be on the order of a few nanoTeslas which increases the necessity of precise measurements.

\subsubsection{Research Objectives}

The ultimate objective of the research described in this thesis is to maximize the achievable resolution of the GeoSurv II magnetic measurement system; this is accomplished by minimizing the magnetic fields produced by the aircraft. The task of minimizing the magnetic field contribution of the actuating system can be broadly divided into two non-mutually exclusive tasks:

- Select non-magnetic (or minimally magnetic) actuators for use on GeoSurv II. 
- Shield or attenuate undesirable magnetic fields. Attenuation means physically cancelling the field by means of superposition as opposed to shielding methods that absorb or redirect the field. This can be achieved through:

- Passive attenuation for time-invariant fields, or

- Active attenuation for time-variant fields.

It is possible that, even with careful selection of minimally-magnetic actuators, there will be some remaining magnetic interference. Furthermore, although the flight control actuating system is expected to be a primary contributor to magnetic interference, the aircraft will also be equipped with an engine, generator. lighting, and electronics/avionics systems; all these systems are expected to generate additional interference.

The specific research objectives for this thesis are:

1. Research attenuation strategies for magnetic interference; investigate the suitability of the strategies for use on GeoSurv II.

2. Investigate low magnetic actuation options for use on GeoSurv II.

3. Develop and experimentally verify a modelling strategy for estimating the intensity of magnetic interference generated by GeoSurv II.

4. Develop and experimentally verify a magnetic interference attenuation strategy.

\subsubsection{Research Contributions}

The following research contributions are presented in this thesis:

1. A novel dipole equivalence model (DEM) for magnetic fields called a Modified Dipole Ellipse Model (MoDEM) is presented and experimentally validated. The 
MoDEM model is experimentally verified for permanent magnets and servo actuators. The algorithm is slown to be superior to the commonly used DEM strategies in the literature.

2. An optimization strategy for minimizing the magnetic interference affecting magnetic sensors is proposed. The algorithm, called Optimal Field Attenuation (OFA), considers the relationship between the configuration of magnetic system components and their combined magnetic fields. Using MoDEM models of these system components a configuration is calculated which minimizes and spatially-stabilizes the average interference measured over an area surrounding magnetically sensitive electronics.

3. The OFA algorithm is experimentally validated for a number of system configurations using permanent magnets.

4. For GeoSurv II, the extension of the OFA algorithm is proposed for minimizing the magnetic interference at two locations and ensuring that the magnitude of the interference is equalized at both locations.

\subsubsection{Outline}

This section outlines the contents of subsequent chapters in the thesis.

\section{Chapter 2: Theory}

The definitions and distinctions between different sources of magnetic fields are described, as is the mathematical basis for dipole equivalence modelling. A literature review of existing dipole equivalence modelling strategies is presented as well. 


\section{Chapter 3: Low Magnetic Signature Actuating System}

This chapter presents a literature survey of low magnetic signature actuators and field attenuation strategies for use on GeoSurv II. The decision to select servo actuators over theoretically lower magnetic signature alternatives is presented. It was concluded that traditional field attenuation strategies, including magnetic shielding and active attenuation systems are unsuitable for use on GeoSurv II. OFA is introduced as a theoretical alternative to traditional shielding strategies.

\section{Chapter 4: Measuring Magnetic Fields}

The development of a magnetic measurement system is described. The test algorithms developed for measuring the magnetic field and parameterizing actuating system components are presented. An analysis of the achievable measurement resolution of the system is discussed.

\section{Chapter 5: Magnetic Field Modelling}

The mathematical formulations of the standard magnetic dipole model described in the literature review (Chapter 2) and the novel MoDEM model are discussed. Both models are used to represent ceramic bar magnets as well as a Futaba FP-S148 and Hitec HSR-5995TG servo. The MoDEM formulation and parameterization algorithm is shown to be superior to the standard modelling strategy and is selected for use in OFA.

\section{Chapter 6: Optimal Field Attenuation}

The Optimal Field Attenuation algorithm is described in detail. A verification experiment involving a set of three MoDEM modelled magnets is presented. The results of the experiment clearly show that both the modelling aspect of the algorithm, as 
well as the prediction of field attenuation are valid and quite accurate. Investigations into specific OFA characteristics and the suitability of the algorithm for minimizing fields on GeoSurv II are also discussed.

\section{Chapter 7: Conclusions and Recommendations}

The research conclusions are listed; recommendations for improvements and future investigations are provided. 


\section{Chapter 2}

\section{Theory}

\subsection{Magnetic Fields}

\subsubsection{Field Strength vs. Magnetic Flux Density/Magnetic Induction}

The magnetic field strength, $\mathbf{H}$, is measured in amperes/meter. In this thesis the magnetic field strength is considered to be solely linked to the magnetizing current.

The magnetic induction (flux density), B, is measured in Teslas (T). The magnetic field produced in a given medium will generate magnetic flux; the concentration, or density, of the flux is a measure of the intensity of the field. The magnetic induction is directly related to the magnetic field by the magnetic permeability $(\mu)$ of the medium, measured in Henrys/meter. This relationship is not necessarily linear; the permeability of many materials exhibit hysteresis [2-5].

A constant magnetic field $(\mathrm{H})$ will generate different magnetic induction fields $(\mathrm{B})$ depending on the medium. However, in this thesis the magnetic field in air will be considered exclusively. In this case the magnetic field and the flux density are related by the permeability of free space $\mu_{0}$ which linearly relates the two. Furthermore, 
measurements of the magnetic field are actually measurements of the flux density [6]. Any further statements discussing the magnetic field will be referring to the magnetic flux density specifically.

\subsubsection{Magnetic Interference}

For this thesis, magnetic interference is defined as any disturbance to the naturally occurring magnetic field of the Eartl.. In the presence of ferromagnetic materials, electromagnets, or permanent magnets, distinguishing between interference and natural envirommental fields is difficult.

The frequency of a source of magnetic interference must also be considered. Extremely low frequency fields, that is below $60 \mathrm{~Hz}$, but more specifically fields that exist within a frequency band of $0-5 \mathrm{~Hz}$ are of primary concern on GeoSurv II. Higher frequency fields may be filtered through data processing algorithms. For this study a source of magnetic interference is defined as any one of the following:

- Ferromagnetic Materials

- Permanent Magnets

- Electromagnets

\section{Ferromagnetic Materials}

Ferromagnetic materials readily magnetize in the presence of an external magnetic field. This classification of ferromagnetic materials includes, but is not restricted to iron, many steels, nickel, cobalt, and their alloys. Ferromagnetic materials are characterized by a high relative magnetic permeability $\left(\mu_{r}\right)$ which is a measure of the extent to which a material magnetizes in the presence of a magnetizing field [2]. 
The magnetic structure of ferromagnetic matcrials is organized into a collection of magnetic domains; each domain consists of some number of aligned magnetic dipoles. These dipoles are initially in a random configuration such that, when combined, results in zero net magnetic field. In the presence of an external magnetic field, the domains align with the direction of the field and the material is said to be magnetized [2]. Materials with high magnetic permeability are high-intensity field sinks; they attract and contain the magnetic field. This disturbs the natural gradient of the field resulting in measurement inaccuracies. On GeoSurv II these materials can be found in DC motor casings (in servos), the aircraft engine, and the power generator.

\section{Permanent Magnets}

In the case of permanent magnets the alignment of magnetic domains does not revert to a random configuration; the material therefore retains its magnetization [2] and is considered a source of magnetic interference. Permanent magnets impart a static addition to the local magnetic field. In the case of rotating electromechanical machinery it is possible that the magnetic field can be cyclically variant. In GeoSurv II these may be the permanent magnets in the DC motors and the power generator.

\section{Electromagnets}

Electromagnetism refers to the relationship between moving electrical charge (currents) and the associated and proportional magnetic field surrounding it $[2,3,5]$. Two sources of interference result from electromagnetism: electromagnetic fields and induced eddy currents.

Electromagnets, in a general sense, refer to magnets produced by any current carrying conductive medium; on the GeoSurv II aircraft electromagnetic elements are 
considered to be either runs of current carrying wire or engineered coil structures designed to produce specific magnetic fields. The latter category includes the windings in electric motors or transformers, solenoids, or toroids to name a few.

Variations in magnetic fields produced by electromagnetic devices is dependant on the current; variable current results in a non-stationary magnetic field. By the same effect that produces electromagnets, any conductor which is moving with respect to a magnetic field will undergo magnetic induction. In conductive materials, induction may generate eddy currents, which are current loops on the surface of a material. These loops create a secondary magnetic field in opposition to the inductive field [5].

\subsection{Modelling Magnetic Fields}

\subsubsection{The Biot-Savart Law}

The Biot-Savart Law describes the relationslip between current and the associated magnetic field [5]. The differential form of the Biot-Savart Law for constant current in a wire is,

$$
d \vec{B}=\frac{\mu_{o}}{4 \pi} \frac{I d l \times \vec{r}}{\|\vec{r}\|^{3}}
$$

In the above equation $d \vec{B}$ is the differential magnetic field at position $\vec{r}$ measured from a differential length of wire, $d l$, with current $I$. The geometry is described in Figure 2.1. Similarly, the Biot-Savart Law can describe the field produced by current in a thick conductor by replacing the current times a differential length $(I d l)$ with the current density times a differential volume $(J d V)$. 


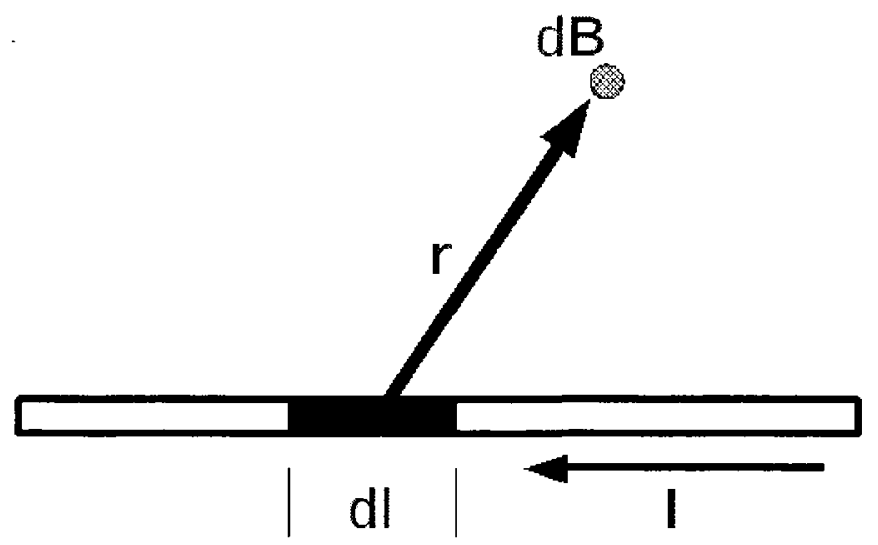

Figure 2.1: Biot-Savart Law. Geometry for Equation 2.1

For circuits that are more complicated than circular loops or when solving for the field at an arbitrary position relative to the circuit, Equation 2.1 is difficult to solve analytically. With the exception of current-carrying wires, or fundamental electromagnetic devices such as solenoids, and toroids, the Biot-Savart Law is not particularly useful for modelling magnetic fields generated by electromechanical devices because the derivation becomes increasingly difficult. The problem can be simplified by considering sources as magnetic dipoles as follows.

\subsubsection{Magnetic Dipole}

Maxwell's equations are a set of equations that govern electronıagnetism [5]. Gauss' Law for Magnetism,

$$
\vec{B}=\nabla \times \vec{A}
$$

where,

$$
\vec{A}=\frac{\mu_{o} I}{4 \pi} \oint \frac{d l}{\|\vec{r}\|}
$$

considers the magnetic field, $\vec{B}$ to be equal to the curl of the magnetic vector potential $\vec{A}$. 
Without proof, the vector potential can be expressed in a more tidy form by introducing the magnetic dipole moment, $\vec{m}$, so that 2.3 simplifies to the form,

$$
\vec{A}=\frac{\mu_{0}}{4 \pi} \frac{\vec{m} \times \vec{r}}{\|\vec{r}\|^{3}}
$$

wliere

$$
\vec{m}=\frac{I}{2} \oint \vec{r} \times d l
$$

The dipole moment is a vector which, essentially, defines the orientation and intensity of a magnetic source. Considering the source of the field to be small with respect to the position of the point of observation [5] allows for the use of an equivalent dipole for representing a magnetic field. In the case of a current loop, this means that the size of the loop is small as compared to the distance to the modelling point. Equation 2.5 can be further simplified when calculating the dipole moment of planar current loops as,

$$
\vec{m}=A I
$$

and for solenoids as,

$$
\vec{m}=n A I
$$

where $A$ is the area enclosed by the current loop, $I$ is the current and $n$, in the case of solenoids, refers to the number of concentric loops. The direction of $\vec{m}$ is parallel to the plane of the loop.

Evaluating Equation 2.2 using the dipole moment form of $\vec{A}$ results in the expression for the dipolar magnetic field,

$$
\vec{B}=\frac{\mu_{o}}{4 \pi}\left[\frac{3(\vec{m} \cdot \hat{r}) \hat{r}-\vec{m}}{\|\vec{r}\|^{3}}\right]
$$


As opposed to the Biot-Savart Law which is a direct relationship between current and magnetic field, using a dipole represcntation of the magnetic field can allow for modelling of many sorts of magnetic sources by calculating or estimating the dipole moment vector [5]. - Dipole equivalence modelling (DEM) is a lumped parameter approach to modelling magnetic fields.

\subsubsection{Dipole Equivalence}

A dipole equivalence model assumes that the magnetic field generated by any device can be represented by a point-source equivalent dipole. A numerical model based on one or more equivalent magnetic dipoles will be developed to represent sources of interference on the GeoSurv II aircraft. The primary focus of the researclı described in this thesis will be the interference generated by servo actuators located in the wing and empennage due to their significant effect on the magnetometers.

A literature review on dipole equivalence strategies for modelling magnetic fields was completed. Several autliors advocate the method for simulating magnetic interference in order to estimate the effectiveness of shielding enclosures or estimating field intensity [7-10]. This section will present the dipole modelling formulations considered for GeoSurv II, and discuss the challenges and limitations of the dipole equivalence approach.

\section{Magnetic Dipole Field}

Figures 2.2 and 2.3 illustrate some standard characteristics associated with dipole magnetic fields. In Figure 2.3 measurements of the magnetic field intensity (magnitude $\|\vec{B}\|)$ were simulated at a constant radial distance from the source of interference, separated by $15^{\circ}$ arc increments. This is equivalent to measuring at a constant position and rotating the source in the plane of the measurement point. These figures 
illustrate what will be referred to as ideally dipolar feld characteristics. The magnitude of the interference was normalized by the maximum value to illustrate that in an ideal case the field oscillates between a maximum intensity of $\|\vec{B}\|$ and a minimum of $0.5\|\vec{B}\|$.

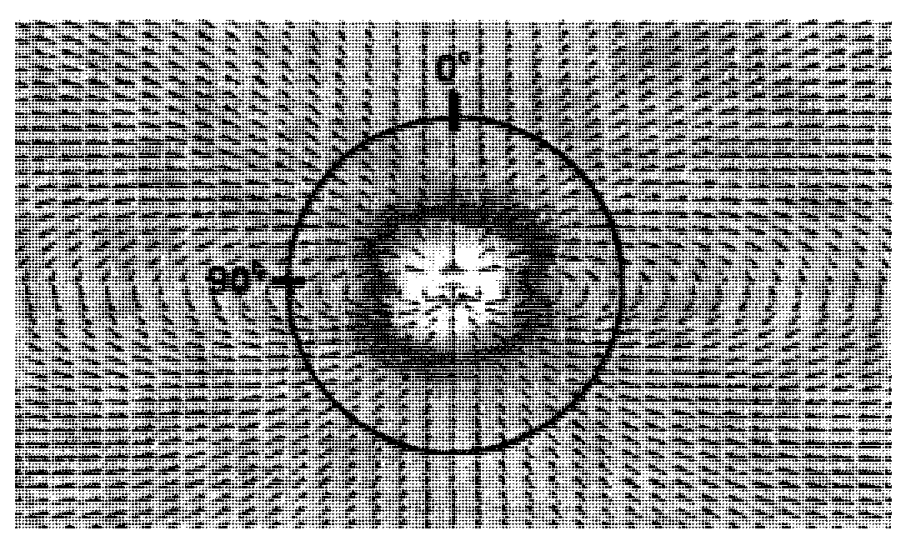

Figure 2.2: Simulated Point-Dipole Magnetic Field. Lines of magnetic flux are illustrated as vectors which form closed paths through the centre of the point-dipole.

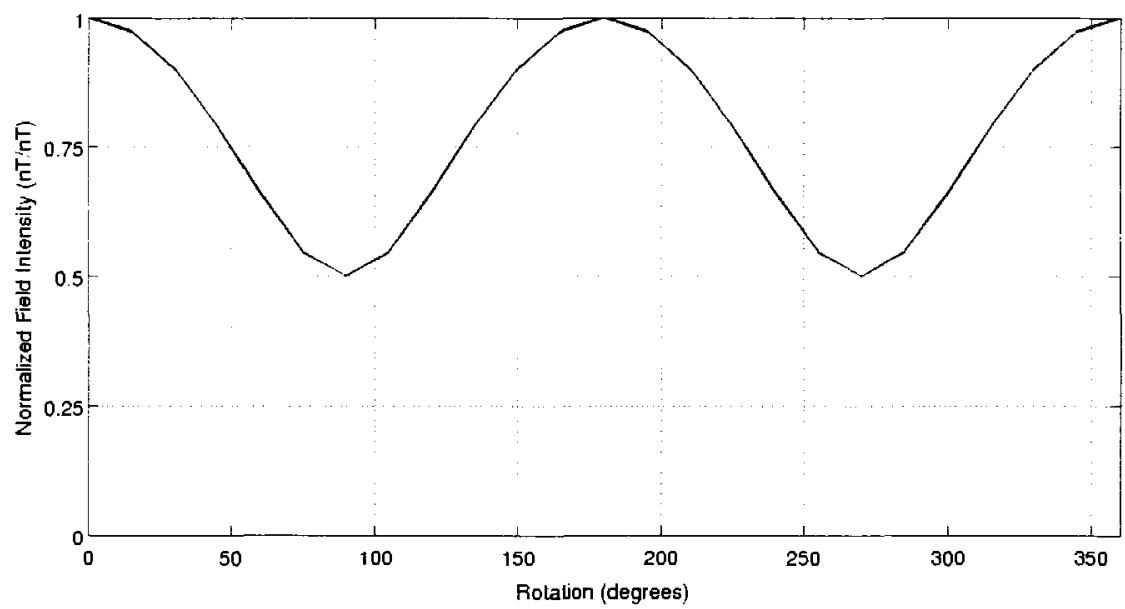

Figure 2.3: Normalized $\vec{B}$ vs. Rotation for a Point-Dipole. The field magnitude measured on the circle shown in Figure 2.2 illustrates an ideally dipolar field characteristic. 


\subsubsection{Survey of Equivalent Dipole Modelling Strategies}

A literature review yielded several examples which not only provided support for the use of dipole equivalence modelling as part of the optimal field attenuation method, but also guidelines and procedures for estimating an equivalent dipole moment for a source of magnetic interference. Modelling strategies described in [7-9] form the foundation of the equivalent dipole analysis methods used in this thesis. Central themes and concepts include the assumption that mechanical devices may be modelled as point dipoles provided that the separation between field source and observation point $(\vec{r})$ is sufficiently large and that the contribution of many magnetic elements in close proximity to one another may be combined into a single model; this is alternatively referred to as far-field analysis.

The application of multipole (higher order modelling basis) equivalence for modelling the environmental magnetic field intensity and distribution with only minor knowledge of the types of field sources was presented in [8]. Two multipole functions were expressed for spherical and cylindrical field sources; using these primitive shape functions the environmental field could be predicted sufficiently to analytically assess shielding strategies. The sorts of objects being modelled included transformers and power lines. To better model these types of sources, the multipole functions were modified to cylindrical or spherical form to better model the shape of the magnetic field surrounding the source being modelled.

Far field analysis methods were demonstrated in $[7,9]$ for modelling household electromechanical devices. Mechanical devices consist of multiple sources of magnetic fields, as well as ferromagnetic materials which distort the local background field. The combined interference exhibits non-dipolar field characteristics close to the device. It is discussed in $[9,10]$ that at some distance measured radially from the device the 
field begins to assume a dipole configuration. These approaches cannot be used in the near field region as the field characteristics are less predictable; a more complex modelling strategy $[10]$ would be necessary. The distinction between near field vs. far field analysis of magnetic fields, as considered in this thesis, was described for the modelling of interference on spacecraft [10]. This thesis considers only far field analysis; that is the region in which the magnetic field is dipolar.

Two strategies were presented for estimating the inagnetic dipole moment. A curve fitting approach [9] mav be used in which the dipole parameters are selected to match magnetic field magnitude and phase measurements taken at several points of known position relative to the source. A simplified approach was described for cases where only the magnitude of the magnetic field must be estimated [7]. The magnetic field surrounding a point dipolc oscillates between a maximum intensity of $\left\|\vec{B}_{\max }\right\|$ and a minimum of $\left\|0.5 \vec{B}_{\text {max }}\right\|$ (Figure 2.3 ) when measured at a constant radial distance. To account for the variance between maximum and minimum intensity of the field a constant,

$$
k=\frac{\left\|\vec{B}_{\text {max }}\right\|}{\left\|\vec{B}_{a v g}\right\|}=1.38
$$

was derived where the average value of the magnetic field, $\left\|\vec{B}_{\text {avg }}\right\|$ on a sphere of constant radius encircling the source, was normalized by the maximum and then inverted.

Although the scalar estimate of the dipole moment is unsuited for the modelling requirements of the optimal field attenuation strategy, it is possible to extend the scalar methodology to a vector estimate rather than applying more complicated curve fitting algorithms for estimating the dipole moment. The costs and benefits associated with curve fitting vs. direct estimation of the dipole moment are trends that will be explored, in detail, in Chapter 5. 


\subsection{Geomagnetism}

The magnetic field of the Earth is often generalized as being a dipole field centered at the core. In reality the magnetic field is neither spatially or time static. Variations in spatial field averages range from 60.000 to $30,000 \mathrm{nT}$ between the poles and equator. Similarly, daily field variations can range between 20 and $100 \mathrm{nT}$ from mid-latitudes to equatorial latitudes (not including the effects of Solar disturbances) [3]. Geomagnetic surveys seek to measure and characterize variations in the magnetic field at the subkilometer level; these variations may be indicators of mineral, oil, or other geological items of interest.

\subsubsection{The Earth's Magnetic Field}

\section{Main Field}

The magnetic field of the Eartl is caused by currents within the molten iron core. Field lines extend from the magnetic south pole towards the magnetic north pole; this is measurable using a simple compass. This main field is dynamic; the positions of the poles are constantly shifting, however these changes are both slow and occur over large areas. Main field changes are readily predictable.

\section{External Field}

Space effects, such as the interaction between solar wind and the ionosphere, produce an external field. This extermal field produces the northern (and southern) lights and can be quite significant in terms of its magnitude. For example, as the Earth spins, diurnal changes in the external field are measured because the incidence of solar wind at a particular location changes accordingly. Furthermore solar winds are not constant. Rather the intensity of solar winds change as a result of changes in solar activity. These effects are often forecasted by studying solar activity; they may 
be avoided by simply not surveying on days when the external field is particularly variable.

\section{Magnetic Anomalies}

Ferromagnetic materials below the surface of the Earth produce regional variations in the measured magnetic flux density. Changes in the flux density over small areas are indicators of the presence (or absence) of magnetic materials. 


\section{Chapter 3}

\section{Low Magnetic Signature Actuation System}

\subsection{Introduction}

The objective of the research project described in this thesis is to maximize the functional resolution of the GeoSurv II magnetometers. Interpreted more generally, the goal of the research is to develop a strategy such that no magnetic interference is perceivable by the aircraft magnetic sensing system. This section presents a review of possible low magnetic actuation schemes for use on GeoSurv II as well as shielding strategies that can be employed to reduce the level of magnetic interference at the sensors.

\subsection{Low Magnetic Actuators for GeoSurv II}

Servo actuators are commonly used in mobile robotic applications. These devices are commercially available with specifications (size, weight, performance) that fit nearly any application. For GeoSurv II servo actuators are the de-facto choice for flight control as they are easily integrated into both the mechanical design of the 
aircraft as well as the avionics system. Flight control is administered through an MP2028 autopilot based control system which is designed for standard digital servo communications [11]. On the other hand, electromechanical actuators may be unsuitable for use on the GeoSurv II because of the inherent magnetic interference produced by such devices. Before making a selection on the actuating scheme for the aircraft a survey of lower magnetic signature alternatives was completed to determine whether or not a better alternative is available. This survey is highlighted in the following section.

\subsection{Survey of Alternative Actuation Schemes}

\subsubsection{Fluid Power Actuation}

Hydraulic actuation systems are commonly used for flight control actuation in the aerospace industry. Magnetic components in a hydraulic system may be reduced by sourcing cylinders, tubing, and valves constructed from non-magnetic materials. Many hydraulic system suppliers cater to much larger systems than GeoSurv II which may necessitate custom component design and integration to ensure that the system remains light-weight.

Pneumatic systems use compressed gas (air) as the working fluid; principal system components are similar to hydraulic requirements. There are several commercial pneumatic suppliers that produce components of the appropriate size for the GeoSurv II actuation system. The principal design challenge for a pneumatic actuation system is to overcome the inherent bandwidth limitations and backdrivability caused by the compressibility of the working fluid. Research into advanced nonlinear controllers and/or design modifications will be necessary for a pneumatic actuation system. 
One such design modification [12] describes a potential application using a bangbang control system in which precise positioning of pneumatic actuators is not necessary. In this case standard flight control surfaces are replaced with a series of independently actuated smaller structures that extend along the entire trailing edge of the airfoil. The Miniature Trailing Edge Effectors (MTEE), Figure 3.1, are independently actuated at angles nearing $+/-90^{\circ}$ thereby avoiding the need for complex pneumatic control systems.

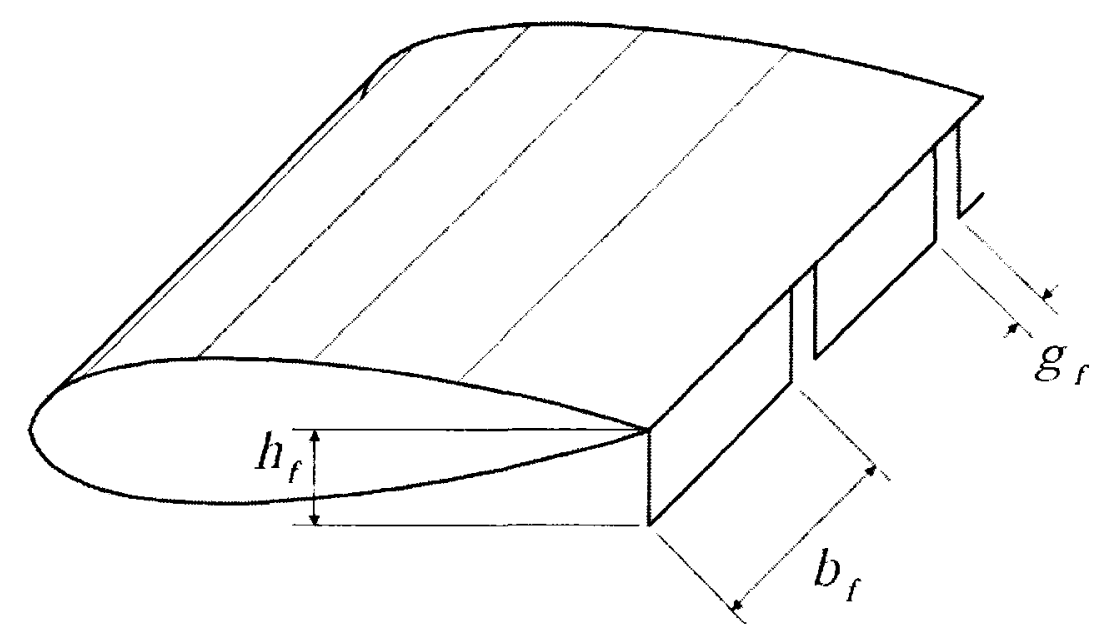

Figure 3.1: Miniature Trailing Edge Effectors. [12] The effectors are sized such that $h_{f}$ measures less than $5 \%$ of airfoil chord. The number of MTEE structures, and their sizing, would depend on GeoSurv II requirements.

\subsubsection{Solid State Actuators}

Solid state actuators refers to a class of materials which strain in response to physical stress (or vice versa); classifications of such materials include:

- Electrostrictive: physical strain when subjected to an electrical field gradient

- Magnetostrictive: physical strain when subjected to a magnetic field gradient 
- Shape Memory Alloy: physical strain when subjected to a thermal gradient (often. through electric current)

Magnetostrictive materials are not suitable for use on GeoSurv II by nature of their actuating principle. Shape memory alloys are not considered because they have limited actuation bandwidth [13].

As electrostrictive materials require the application of a higher ratio of voltage to current than traditional electric motors, it is conceivable that such materials may be suitable for low magnetic actuation applications. Piezo ceramics are a common class of materials used to actuate structures electrostrictively. Individual piezo elements have very high specific strengths but micrometer level displacements [14]. The use of piezo ceramic actuators on GeoSurv II may be possible by developing an actively controlled wing structure or by developing structures to amplify piezo displacements [15-18]. Such a solution would require extensive research and development beyond the scope of this research project.

\subsubsection{Hybrid Actuators}

It is possible to mechanically leverage the specific strength of piezo actuators to achieve increased control displacement. Some strategies include:

- Inchworm Actuators

- Precompressed Piezo Actuating Elements

- Hybrid Piezo/Fluid Actuators

\section{Inchworm Actuators}

Inchworm Actuators $[14,19]$ are devices in which several piezo elements are configured such that axial motion is produced by a coordinated cycle of clamping and 
extending operations. Figure 3.2 illustrates a generic incling motor process in which a shaft is actuated by a sequence of three piezo elements. Alternatively, inching motors can be designed to crawl along a fixed shaft.

Inchworm drives are relatively new devices; there are few commercial suppliers. Most designs are in a developmental stage, and their suitability in an aircraft control capacity has yet to be determined. Those that are currently available on the market are designed for the optics/photonics industry which requires micrometer precision and short actuator strokes. The shaft speed on inchworm actuators is usually slow compared to traditional linear servo, or hydraulic actuators. An advantage of the design is that it is self-locking; no power is necessary to prevent backdriving.

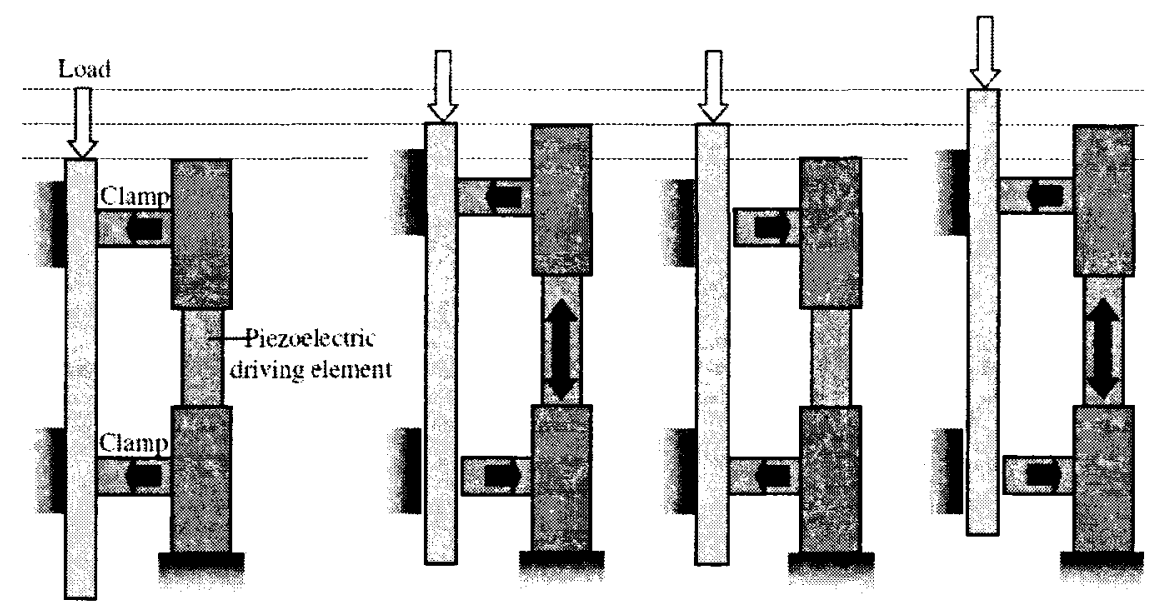

Figure 3.2: Inchworm Operation. Two clamping elements (one fixed and the other free) and an extending element are triggered in sequence to achieve piecewise smooth translation of a shaft [14].

\section{Precompressed Piezo Actuating Elements}

Precompressed piezo actuating elements (PCAE) are effectively shape controlled beams [16]; the deformation is used to change the camber of a wing in a similar fashion to conventional control surfaces; however it is the control surface itself that 
structurally deforms, or morphs locally. It is possible to develop a stand-alone morphing control surface module, Figure 3.3, [17,20] that can be initegrated into the GeoSurv aircraft. Such a system could be highly integrable with the GeoSurv II wing because there would be no need to construct fairings to hold actuators, or to pass mechanical linkages through the wing. A PCAE control surface would only require power and control wiring.
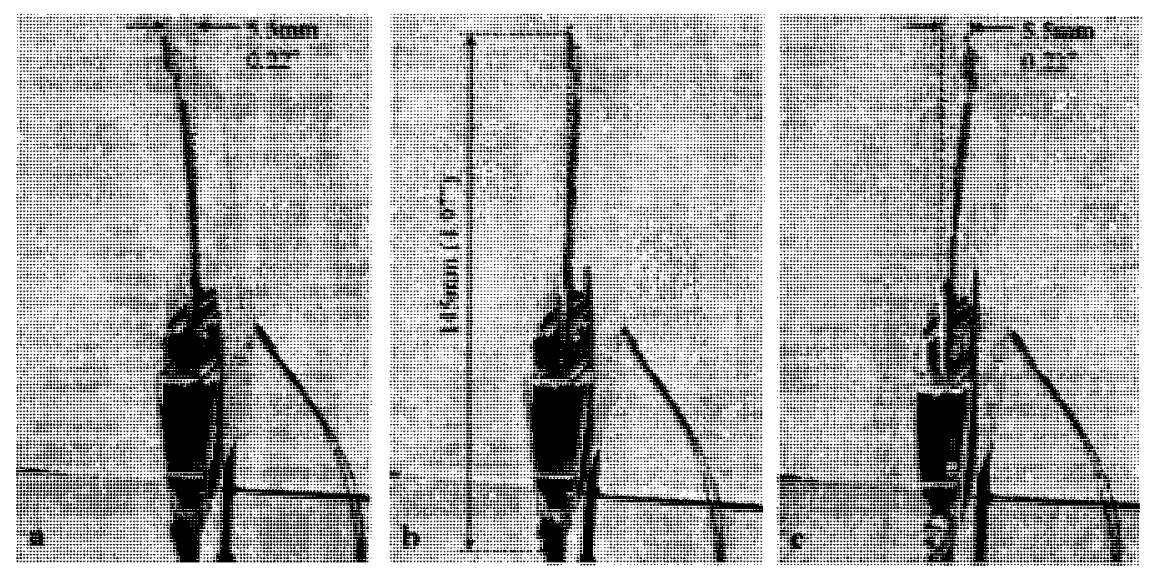

Figure 3.3: Post-Buckled Precompressed (PBP) Control Surface. Prototype of a PBP control surface developed at TU Delft [20].

\section{Hybrid Piezo/Fluid Actuators}

Development of piezo actuated hydraulic pumps used to produce a smart materialbased electro-hydrostatic actuator (EHA) [21,22] may also be possible on GeoSurv II. EHA actuators are self contained hydraulic actuator units (pump, fluid, piston, and valving) currently being developed for, and used in, large-scale commercial and fighter aircraft; these devices have the benefits of hydraulic actuating systems (high force-to-weight ratio, bandwidth, etc...) without the need for central fluid reservoirs and hydraulic lines. The devices that were reviewed are still in academic research and development; in the future such devices may represent ideal actuation candidates for mid-sized UAV's such as GeoSurv II. 


\subsubsection{Flapless Control}

Traditional aircraft flight control is achieved by deflecting flight control surfaces (elevator, rudder, aileron, etc...) in order to affect the airflow over a wing to produce an aerodynamic moment (pitch, yaw, roll, etc...). Flapless control refers to methods of controlling an aircraft without mechanically deploying or actuating a control surface. The FLAVIIR (Flapless Air Vehicle Integrated Industrial Research) Project [23] has successfully flight tested a flapless RC aircraft using only forced air. The configuration is illustrated in Figure 3.4. A similar approach is proposed for general aviation aircraft using a double slotted pulsed pneumatic flap to redirect the primary airflow over the wing as if a physical control surface had been deflected [24-27].
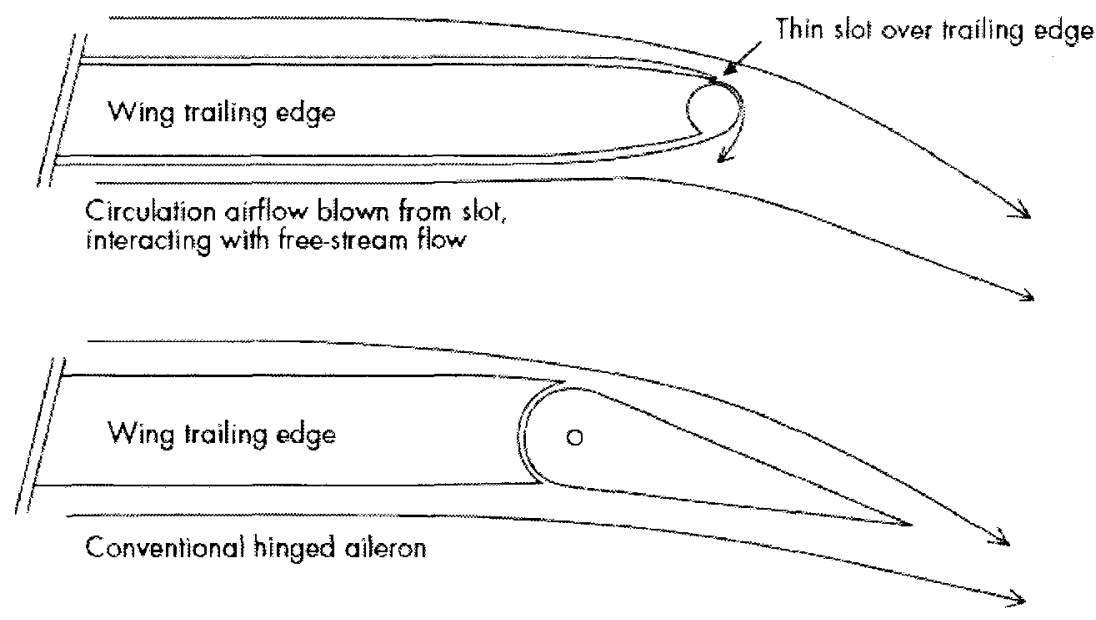

Figure 3.4: Flapless Airfoil Illustration. [23]

Implementing a flapless control system on GeoSurv II would require extensive modification to the structural configuration of the aircraft and the introduction of novel design methods. Traditional aircraft sizing methods and heuristic design aids are incompatible with such a control scheme. A pressurized air delivery system including compressor and valve hardware would also be required. As with the pneumatic 
system discussed in Section 3.3.1, control valves may produce significant magnetic interference.

\subsubsection{Actuator Selection}

When the actuating system survey was completed, the design team of GeoSurv II had not yet determined specific actuating system performance requirements such as torque, rate, or bandwidth for the various flight control surfaces. A decision on the class of actuator for use on GeoSurv II was made on the basis of qualitative criteria such as the ability to integrate the system into GeoSurv II, theoretical magnetic field effects, and control requirements. The actuator comparisoll matrix is in Appendix A. In many cases, such as the piezo hybrid and solid-state actuation strategies or flapless control, the actuation concepts had not yet reached commercial availability which would require in-house research, development. and validation for use on GeoSurv II.

It was determined that the best course of action, considering the parallel development of the GeoSurv II aircraft along with the research discussed in this thesis, would be to proceed with the selection of DC servo actuators. Although it is quite conceivable that other actuators identified in the survey would be better suited in terms of magnetic interference levels, they would require, to varying extents, significant changes in the aircraft design. The decision to proceed with electromagnetic servo actuators for GeoSurv II confirms the necessity to implement a magnetic field attenuation strategy in order to ensure that magnetic field requirements are met.

\subsection{Strategies for Minimizing Magnetic Fields}

The nomenclature used in this thesis differentiates magnetic minimization strategies on the basis of the mechanism through which they reduce magnetic interference. The 
following two classifications are used:

1. Passive Tecluniques, and

2. Active Techniques.

\subsubsection{Passive Techniques}

Passive techniques refer to methods that fall under the more traditional term magnetic shielding. In general these methods achieve field minimization through the application of a physical barrier to the propagation of magnetic field. Factors that affect the design of shields include [28-30]:

- Field Intensity

- Field Frequency

- Required Shape

- Shielding Mechanism

For time varying fields (frequency $\neq 0$ ) a layered arrangement of ferromagnetic and conductive (but non-magnetic) materials are often used [31,32]. Ferromagnetic materials are useful for trapping (absorbing) magnetic field; however, depending on the saturation limits, thickness, and number of conductive material layers the shield may have a net characteristic of reflecting rather than absorbing the magnetic field. Conductive materials shield against time varying fields because eddy currents induced in the material generate a secondary magnetic field that is repulsive to the incident interference. 
Static (DC electromagnetic) magnetic interference is more difficult to shield because the eddy current effect cannot be exploited. Shielding of static fields requires more precise selection of ferromagnetic materials to ensure maximum attenuation [28].

Magnetic shields using ferromagnetic materials are unsuitable for use on GeoSurv II. The attractive nature of ferromagnetic materials produces a secondary form of magnetic interference as the field-sinking nature of the material modifies the local field gradient. Secondly, eddy currents produced in conductive materials will generate additional magnetic interference which must be avoided. Additionally, conductive nonmagnetic shielding is not well suited to shield against static magnetic fields.

\subsubsection{Active Techniques}

Active magnetic shielding, attenuation, or cancellation, refers to a strategy in which a dynamically controlled system of electromagnetic coils is used to superimpose a magnetic field of equal intensity but opposite direction such that the resultant field is null. Buccella et al describe an implementation of active shielding in which a cancellation field is generated by an active coil enclosing a shielded region containing a magnetometer [33]. Buccella achieved $25 \mathrm{~dB}$ attenuation in the total field for a field frequency of $50 \mathrm{~Hz}$. The objective on GeoSurv II is to attenuate only the magnetic interference: complex control systems and field models would be necessary to ensure that the environmental field is not affected.

A more complex method called Supershielding [34] was discovered in which concentric cylindrical or spherical shells may be used to shield components within the supershield or to contain magnetic fields produced by sources of interference within it. Such an application may be promising for GeoSurv II as a supershielding enclosure may be integrated into the fuselage to contain all magnetic interference generated by 
the aircraft.

For the case described in this thesis, implementation of active shields requires a detailed understanding of the nature of the magnetic interference generated by the vehicle. The active controller would require sophisticated magnetic field models such that only undesired fields are attenuated, leaving environmental magnetic fields to be measured. For the study described in this thesis, active shielding will not be the principal focus; rather an attemuation strategy with primary focus on static fields will be developed. Future work may extend the research to attenuating time-variant fields.

\subsection{Optimal Field Attenuation (OFA)}

The fundamental approach of an active attenuation system is to generate and superimpose a vector field of equal intensity and opposite sign such that the resultant is zero. A significant proportion of the interference to be attenuated on GeoSurv II will be static. Such interference is expected from permanent magnets in the servo motor stator, DC magnetic fields while holding stationary positions, and miscellaneous ferromagnetic materials distributed through the system. To attenuate such fields using an active system is costly in terms of energy consumption and design effort.

It is proposed that a novel strategy called Optimal Field Attenuation (OFA) be adopted for passive attenuation of magnetic interference. Whereas both classical passive magnetic shielding and active cancellation require additional system infrastructure, the strategy proposed in this thesis involves determining an optimal configuration (position and orientation) of the flight control actuating system components with respect to their combined magnetic signatures. 
Development of an OFA solution for the magnetic interference problem on GeoSurv II will require completion of several tasks, including model algorithm development. validation testing, and mathematical optimization algorithm development and validation. These are discussed in subsequent chapters of the thesis. 


\section{Chapter 4}

\section{Measuring Magnetic Fields}

\subsection{Introduction}

Magnetometers are designed to measure the effects of magnetic fields interacting with some physical meter. The choice of meter differentiates the various magnetometer options. For example, in the case of a magnetic compass the environmental field applies a force on a suspended piece of magnetic material resulting in the material aligning itself with the field. The operational principle for more advanced magnetometers will be discussed in this chapter. The development of an apparatus for measuring magnetic fields produced by GeoSurv II will be discussed as well.

\subsection{Magnetic Anomaly Detection (MAD) Shack Facility}

SGL operates a magnetic testing facility located at the Ottawa International Airport called the Magnetic Anomaly Detection (MAD) Shack. This facility is located in a magnetically quiet (static magnetic field) environment, raised on pillars above the ground and constructed from nonmagnetic materials, to the greatest extent possible. 
SGL uses this facility to measure the magnetic signature of additions to their aircraft and to conduct performance testing on magnetometers. Measurements are taken using several synchronized optically pumped cesium magnetometers; these are the same type of sensors that will be used in the GeoSurv II for airborne geomagnetic surveys. The data acquisition (DAQ) system is capable of collecting data at a resolution of sub $0.01 \mathrm{nT}$ which is the maximum design allowance target for time-variant sources of magnetic interference. Fluxgate magnetometers are also used, in a secondary capacity in the MAD shack to measure the vector field.

\subsubsection{Optically Pumped Cesium Magnetometer}

Sander Geoplysics Limited (SGL) intends to equip the GeoSurv II with two Geometrics G822A optically pumped cesium magnetometers in a horizontal gradiometer configuration. The operational basis of alkali vapour magnetometers involves deducing the magnetic field through its relationship with the effort required to force the cesium atoms to the highest quantum energy level; the exact plysics are beyond the scope of this thesis but can be found in [3]. Optically pumped alkali vapour magnetometers are highly sensitive and can resolve magnetic fields on the order of $0.0005 \mathrm{nT} / \sqrt{\mathrm{Hz}}$. The only drawback is that they measure the magnitude of the field, not the vector.

\subsubsection{Fluxgate (Saturable-Core) Magnetometer}

Although not as sensitive as the cesium magnetometers, SGL also uses vector fluxgate magnetometers such as the Billingsley TFM 100 which was selected for use on GeoSurv II. The fluxgate magnetomer infers the magnetic field through measurement and processing of secondary field harmonics produced by tiny coil antennas 
surrounding highly permeable magnetic materials. Fluxgate magnetometers can resolve magnetic fields on the order of a few nanoTesla [3].

\subsection{GeoSurv II Magnetic Measurement System}

Although SGL has made the MAD Shack facility available for the development of the GS2 low magnetic signature actuating system project, its location at the Ottawa Airport necessitates that an SGL employee with security clearance be present. It is difficult under these circumstances to accurately and carefully develop and complete the testing of modelling and attenuation algorithms. Also, the primary measurement devices at the MAD Shack are scalar magnetometers. The research project discussed in this thesis requires vector measurements of the field. Ultimately it was deemed necessary to develop and commission an in-house magnetic measurement capability at Carleton University. This section will discuss the hardware, software, and characterization work completed in developing the measurement system.

\subsubsection{Hardware Selection}

The Honeywell HMR 2300 Smart Digital Magnetometer (SDM). Figure 4.1 was selected for the measurement system. The SDM is a three-axis (XYZ orthogonal) magnetoresistive magnetometer, equipped with 16bit ADC and is configured for RS232 communications. The sensor is capable of resolving down to $7 \mathrm{nT}$ at a frequency of $154 \mathrm{~Hz}$; this is on the same order of magnitude as similar fluxgate magnetometers. RS-232 serial communications protocol allows for a direct PC to sensor link and avoids the necessity of additional data acquisition hardware. In addition, only 612 unregulated VDC is required to power the sensor which adds the possibility of configuring the sensor as a wireless device for use in GeoSurv II ground and flight testing. 


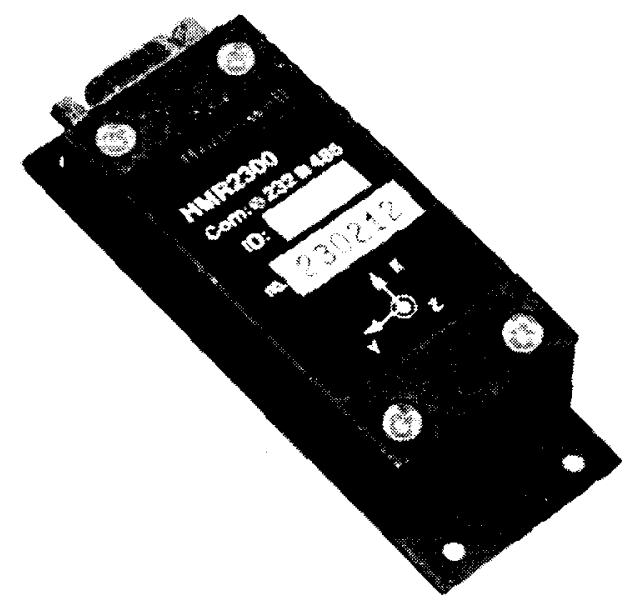

Figure 4.1: HMR-2300 Sensor. The device dimensions are $0.082 \mathrm{~m}$ (length) $\mathrm{x}$ $0.038 \mathrm{~m}$ (width) and $0.022 \mathrm{~m}$ (high); the weight is $0.098 \mathrm{~kg}$.

\subsubsection{Data Acquisition System}

The SDM was designed to communicate through RS 232 serial communications using a set of programmed commands; Matlab was used to communicate with the sensor as well as for data acquisition and processing. There are two principal sensing modes for the HMR 2300, polled (discrete) or continuous sampling. All measurements were taken using the continuous sampling mode. The experiments discussed in this thesis did not require real-time filtering, processing, visualization, or analysis. Therefore in all cases the sensor was commanded to collect for a user-defined period after which the data was extracted from the sensor write buffer, processed, and visualized.

\subsubsection{System Configuration}

Facilities such as the SGL MAD Shack are located in a magnetically quiet location; the magnetic field magnitude in such an area is spatially constant. This characteristic is exploited for measuring the magnetic signature of objects or devices by using one principal sensor in close proximity to the item being measured and a second at a distance sufficiently far away so that no magnetic signature from the object may be 


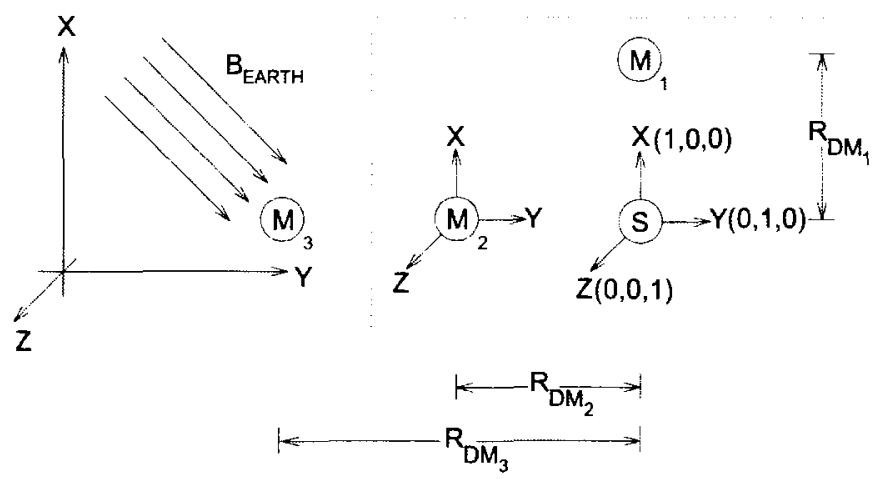

Figure 4.2: MAD Shack Facility schematic. Magnetometers M1 and M3 refer to the primary and offset scalar magnetometers, respectively; M2 refers to the additional vector fluxgate magnetometer used to measure the field source, S. Simultaneous sampling of the field from each magnetometer allows for real-time offsetting of the environmental field and, theoretically, the best functional test resolution.

measured. By synchronizing the measurements, the distant magnetometer can be used to offset the environmental magnetic field from the primary test sensor, Figure 4.2. Such an arrangement was not possible for the measurement system used at Carleton University.

The field in most urban areas (indoors or otherwise) is generally not spatially stable. The presence of ferromagnetic materials, electrical cables, and mechanical devices, among others, produces a much more spatially dynamic field than is experienced in non-urban areas. Secondly, when working with vector magnetometers, the components of the field must be the same between the two sensors in order to accurately normalize the measurements. It was deemed too difficult to configure a two-sensor system. By assuming that the average magnetic field in a testing environment was constant over short time periods, a single sensor apparatus was developed. In effect, a snapshot of the magnetic field is taken pre-test and used to offset the background field from future measurements. 


\subsubsection{Measurement Algorithm}

A custom magnetic testing algorithm was developed to accurately measure the magnetic field of actuation system components using only a single sensor; the Matlab scripts are included in Appendix B. The general test algorithm involves the following steps:

1. Test Environmental Selection and Characterization

\section{Pre-Test Measurement Error Characterization}

\section{Test Data Acquisition}

\section{Posi-Test Measurement Error Characterization}

The first two steps in the measurement algorithm involve characterizing the background magnetic field and determining the functional resolution of the apparatus under the current circumstances. The functional resolution considers the resolution of the entire measurement system including the variability in the background magnetic field, rather than simply considering the resolution of the sensor itself. In a perfectly time-invariant magnetic field environment the functional resolution should be equal to the sensor resolution.

Working with the assumption that the environnental field is static over short periods of time, a script (characterize.m in Appendix B) was developed to characterize and display the actual variance in the field. The script is configured to sample the field at a frequency of $154 \mathrm{~Hz}$ for a user-prompted time period. This data is processed and the sample mean and standard deviation are calculated. This data is analyzed to ascertain the extremely low frequency variance of the field. If the background field is observed to exhibit large mean deviations, the environment is considered unsuitable for testing at that time. 
The second characterization step involves sampling the background field through $\mathrm{N}$ measurement increments averaged for $\mathrm{M}$ seconds each; the mumber of increments and hold times are determined by the user. The Matlab script resolution.m offsets the discretized measurements by the average XYZ field values determined in step 1; if the background mean is entirely static then one would expect that the discretized measurements would all be zero. In this case the achievable data resolution would be $7 \mathrm{nT}$ which is the design resolution of the sensor. In most cases, however, there will be a variation in the measurements; this variation is called the functional resolution of the apparatus for the current test.

The test measurement script (measurement.m) is effectively identical to the resolution.m script however the user is prompted to begin each data acquisition increment. Post test error characterization is used to determine if any significant changes have occurred during the test. If the post test characterization differs from the pre-test values it may be necessary to discard the test data; this decision must be made on a case-by-case basis depending on the error vs. the magnitude of the test values. For example if the pre- to post-test characterization errors differ by less than a few percent of the test measurements, the data is assumed to be good. Pre- to post-test characterization equality is not sufficient, however, to conclude that the mean field was indeed invariant during the test.

\subsubsection{Measurement System Analysis}

The functional resolution of the measurement system is generally dependant on the environmental conditions. Although the HMR-2300 magnetometer has a theoretical design resolution of $7 \mathrm{nT}$, the functional resolution is related to the variation in measurements taken by the system as a whole; this includes the environment. A study was undertaken to determine the relationship between the sampling time for the data 


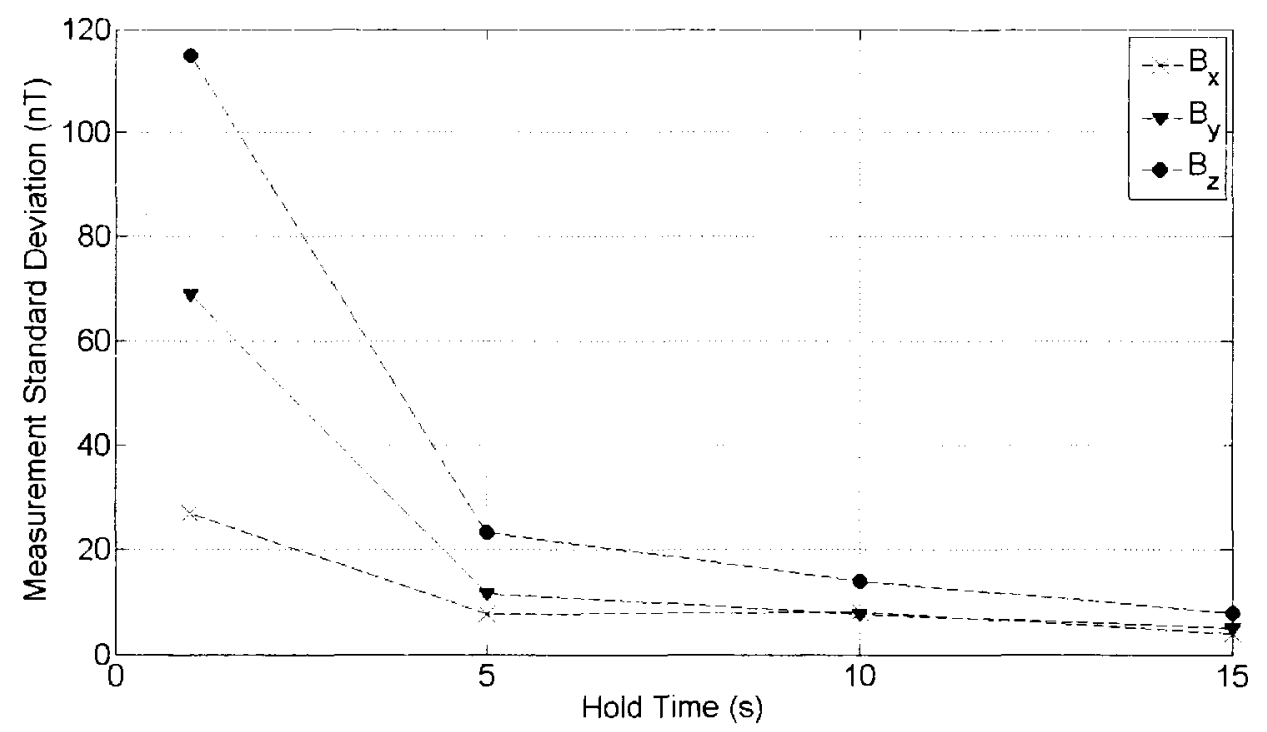

Figure 4.3: Standard Deviation of $\vec{B}_{\text {environment }}$ vs. Sampling Hold Time

acquisition increments and the variation in the measurements. Sampling times of 1 , 5,10 , and 15 seconds were considered; the background field was characterized and 25 measurements were taken.

Using the standard deviation of the 25 increments as a measurement of the functional resolution, it was observed that for all three components of the field, the measurement error decreases with increasing hold time. Between one and five seconds the decrease was observed to be quite significant with a linear relationship between standard deviation and hold time for the remaining three data points. Figure 4.3 shows these trends.

It was decided that a minimum of ten seconds be used for all field testing to ensure the highest quality test data. It must be noted that there is no control over the background field and even using a hold of ten seconds may still result in poor data if the environment changes. To overcome this problem, it is important to periodically check the functional resolution between test cases using the same environmental 
characterization data. If the environment has changed then a new characterization is necessary. 


\section{Chapter 5}

\section{Magnetic Field Modelling}

\subsection{Introduction}

By using an equivalent dipole model it is possible to characterize the actuating system as a collection of point-acting magnetic sources. This chapter will present the development of a model basis for use with the Optimal Field Attenuation strategy of minimizing magnetic interference on the GeoSurv II. Two competing modelling strategies are presented; the standard dipole model as described in Equation 2.8 and a novel approach referred to as a Modified Dipole Ellipse Model (MoDEM).

\subsection{Data Acquisition}

The data acquisition system described in Chapter 4 was used to collect dipole moment parameterization data; the test configuration is illustrated in Figure 5.1. The parameterization test procedure involves the following steps:

1. Coordinate System Assignment. The best-practice is to choose a coordinate system consistent with the mounting hardware, and necessary operational orientation of the object. Ultimately the item must be installed in the aircraft; choosing a coordinate system that is convenient to measure to and rotate about 
will be beneficial to assembling the actuating system in an optimal configuration.

2. Pre-test Environmental Characterization. Sample the environmental background magnetic field and determine the functional resolution for the test case.

3. $\vec{B}$ vs. Applied Rotation Measurement. Sample the magnetic field produced by the test object at desired rotational increments between $0^{\circ}$ and $360^{\circ}$.

4. Post-test Environmental Characterization.

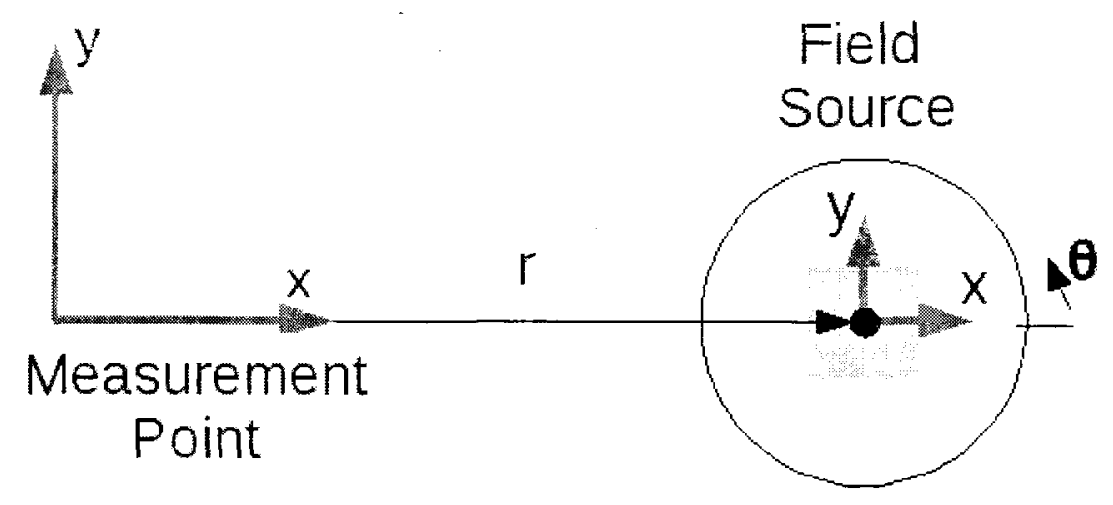

Figure 5.1: Dipole Parameterization Test Schematic

\subsubsection{Test Objects}

Five sources were measured:

- 1 Two-element ceramic dipole magnet (2DP).

- 2 Three-element ceramic dipole magnets (3DPa, 3DPb).

- 1 Futaba FP-S148 Servo Actuator.

- 1 Hitec HSR-5995TG Servo Actuator. 
The 2DP and 3DP specimens were manufactured using ceramic bar magnets; the exact composition of the material is unknown. Because a bar magnet is effectively a dipole source, these sources were expected to provide an excellent starting point and benchmark for verifying the parameterization algorithm and establishing model suitability. Figure 5.2 illustrates the coordinate system assignment for the two dipole sources.
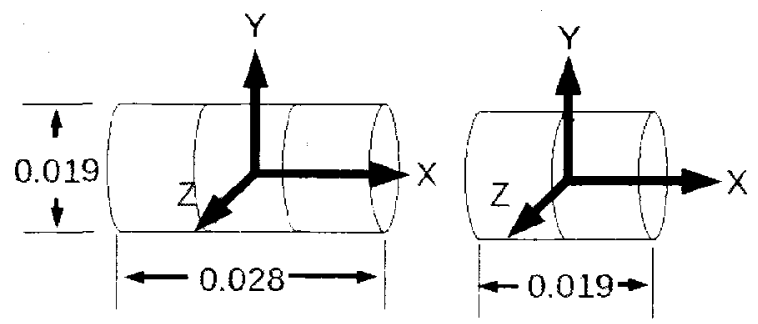

Figure 5.2: Coordinate System Assignment - 2 and 3DP Field Sources (all dimensions in meters)

The Hitec HSR-5995TG servo actuator was selected for use on GeoSurv II. It contains a minimum of ferromagnetic components including a coreless DC motor and titanium gears. The coordinate system assignment for the HRS-5995TG is described in Figure 5.3.
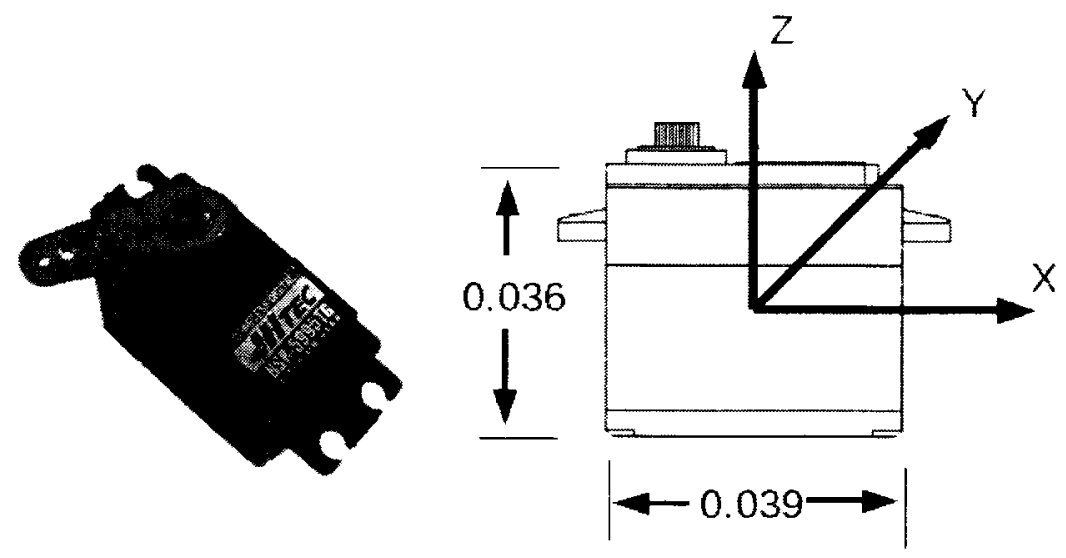

Figure 5.3: Coordinate System Assignment - Hitec HSR-5995TG (all dimensions in meters) 
The Futaba FP-S148 uses nylon gears and a 3-pole ferrite core DC motor; it is of similar external dimensions as the Hitec, lowever it has a lower power motor. The coordinate system assignment for the FP-S148 is described in Figure 5.4.
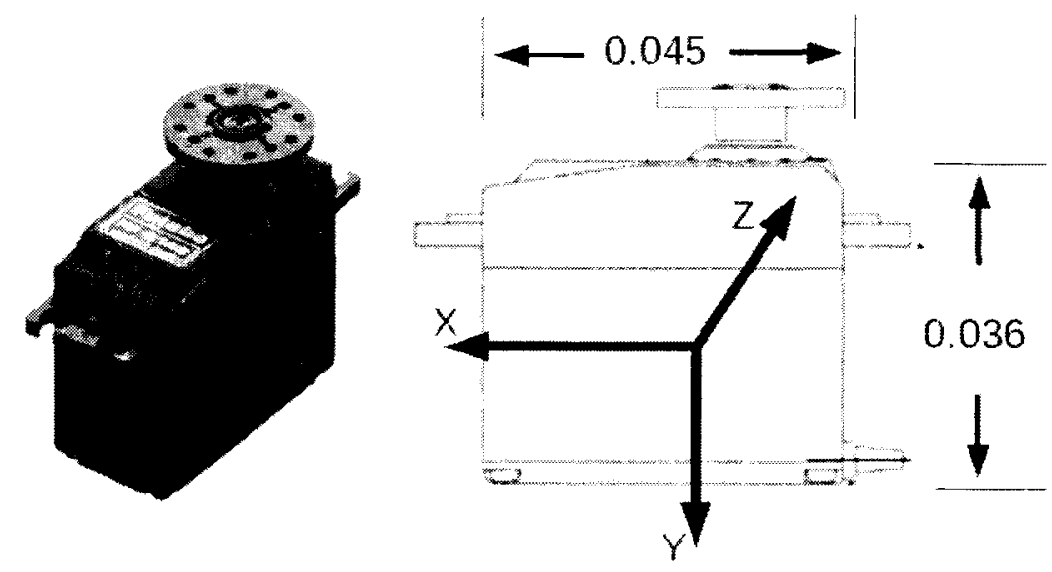

Figure 5.4: Coordinate System Assignment - Futaba FP-S148 (all dimensions in meters)

All specimens were tested at a distance of $0.3 \mathrm{nn}$ measured along the $\mathrm{X}$-axis assigned to the magnetometer with the exception of the Futaba servo which was tested at $0.2 \mathrm{~m}$ separation to improve signal to noise characteristics; these distances are believed to be in the far-field region based on the three tines distance criteria described in [9]. The initial orientation of the specimens aligned the assigned coordinate system with the magnetometer coordinate system. In each case the field was sampled at $154 \mathrm{~Hz}$ with rotational increments of $15^{\circ}$.

\subsection{Dipole Modelling}

Modelling magnetic field sources as equivalent point-acting dipoles requires estimation of the inagnetic dipole $(\vec{m})$. The direct dipole parameterization method (DDP) estimates $\vec{m}$ by solving the dipole field equation based on measurements of the field 
taken at a known distance. Substituting $\hat{r}=\left[\hat{r}_{x} \hat{r}_{y} \hat{r}_{z}\right]^{T}$ and $\vec{m}=\left[m_{x} m_{y} m_{z}\right]^{T}$ into the dipole field equation (Equation 2.8) and evaluating the inner product results in the matrix form of the dipole field equation,

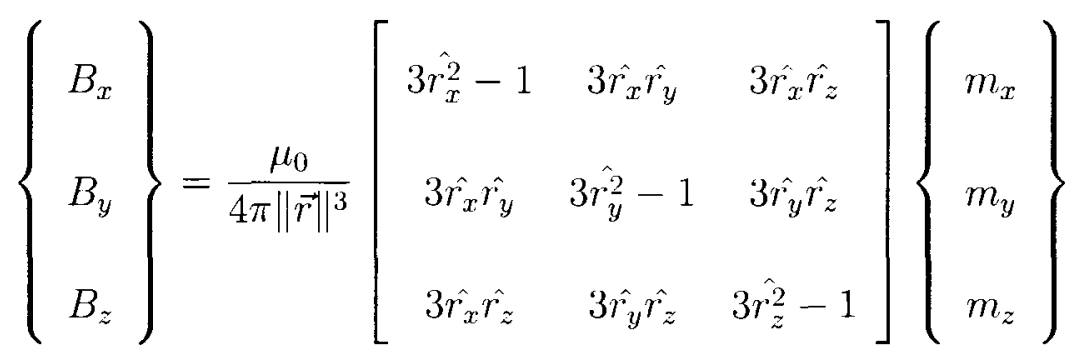

Equation 2.8 relates a measurement of the magnetic field, $\vec{B}$, directly with the dipole moment, $\vec{m}$, of the magnetic source through a system of three linear equations. Assuming that the position of the measurement point is known with respect to the location of the equivalent magnetic source, $\vec{r}$, the dipole moment necessary to produce the field measurement can be quickly calculated.

The relationship between the measured magnetic field vs. applied rotation of a dipole source was described in Section 2.2.3; as the source rotates $B_{x}, B_{y}$, and $B_{z}$ are observed to oscillate between positive and negative maxima and minima of equal absolute value. The magnitude, in an ideal case, oscillates between a $\left\|\vec{B}_{\text {max }}\right\|$ and $\left\|0.5 \vec{B}_{\text {max }}\right\|$ values for increments of $180^{\circ}$ and $90^{\circ}$ respectively. These trends are illustrated in Figure 2.3 in Section 2.2.3. Because this characteristic is highly specific to the dipole field (rather than the more arbitrary $R^{3}$ decay rate that would be observed for linear translation) rotational measurements were selected for parameterization test.ing. 


\subsubsection{Direct Dipole Parameterization}

A script (DDPMethod.m) was developed to estimate the dipole moment using the direct dipole parameterization method. The script takes in the measured values of magnetic field vs. applied rotation and returns an estimate of the dipole moment vector as well as the modelled field vs. rotation curves for comparison. The direct parameterization method uses Equation 5.1 to calculate the equivalent dipole moment vector that would produce each field measurement over the full $360^{\circ}$ rotation of the modelled source. By correcting each subsequent estimate for the applied rotation, a set of theoretical values is calculated; the estimate of the dipole moment is considered to be the average of this set.

In an ideal case this set should be uniform, however in actuality there will be variability among the $m_{x}, m_{y}$, and $m_{z}$ terms for a number of factors which will be discussed in detail in Section 5.5.

\subsubsection{Direct Dipole Test Results}

Table 5.1 lists the absolute mean error between the predicted and measured $\vec{B}$ and $\|\vec{B}\|$ values. Figures 5.5 and 5.6 plot the direct dipole modelled vs. experinientally measured $\vec{B}$ vs. applied rotation for the two and three bar magnetic sources respectively. Figures 5.7 and 5.8 plot the direct dipole modelled vs. experimentally measured $\vec{B}$ vs. applied rotation for the Futaba and Hitec servos.

The results of DDM for bar magnet sources show reasonable agreement with the measured values. For both the 2DP and 3DP sources there was poor modelling of the $B_{z}$ data; possible reasons for this will be discussed in Section 5.5. The error in the $B_{x}$ and $B_{y}$ curves is most likely attributed to an error in positioning the axis of rotation with the central axis of the magnet. 
DDM estimates of the magnetic field produced by the Futaba servo, Figure 5.7, was rather poor. The source was rotated about its geometric centre; this point is likely not coincident with the equivalent magnetic centroid. The result of this offset is that the measured values do not follow an ideally dipolar trend that cannot be aceurately modelled using the DDP/M algorithm. The sawtooth pattern notable on the $B_{y}$ and $B_{z}$ curves is attributed to increased variability in the background magnetic field. The agreement between DDM estimate and measured field values for the Hitec servo, Figure 5.8, was reasonable. As with the Futaba servo, although to a lesser extent, an offset between the point of rotation and the location of the equivalent magnetic dipole explains the the modelling error.

Table 5.1: Mean Absolute Modelling Error (nT) for the DDM/P Field Estimation Algorithm

\begin{tabular}{|c|c|c|c|c|}
\hline Source & $B_{x}$ & $B_{y}$ & $B_{z}$ & $\|\vec{B}\|$ \\
\hline $2 \mathrm{DP}$ & 336.7 & 246.68 & 1029.6 & 208.0 \\
\hline $3 \mathrm{DPa}$ & 462.0 & 367.1 & 1390.8 & 226.0 \\
\hline $3 \mathrm{DPb}$ & 913.3 & 607.3 & 1409 & 444 \\
\hline Futaba FP-S148 & 182.4 & 47.0 & 35.3 & 137.0 \\
\hline Hitec HSR-5995TG & 78.1 & 48.8 & 61.0 & 76.8 \\
\hline
\end{tabular}




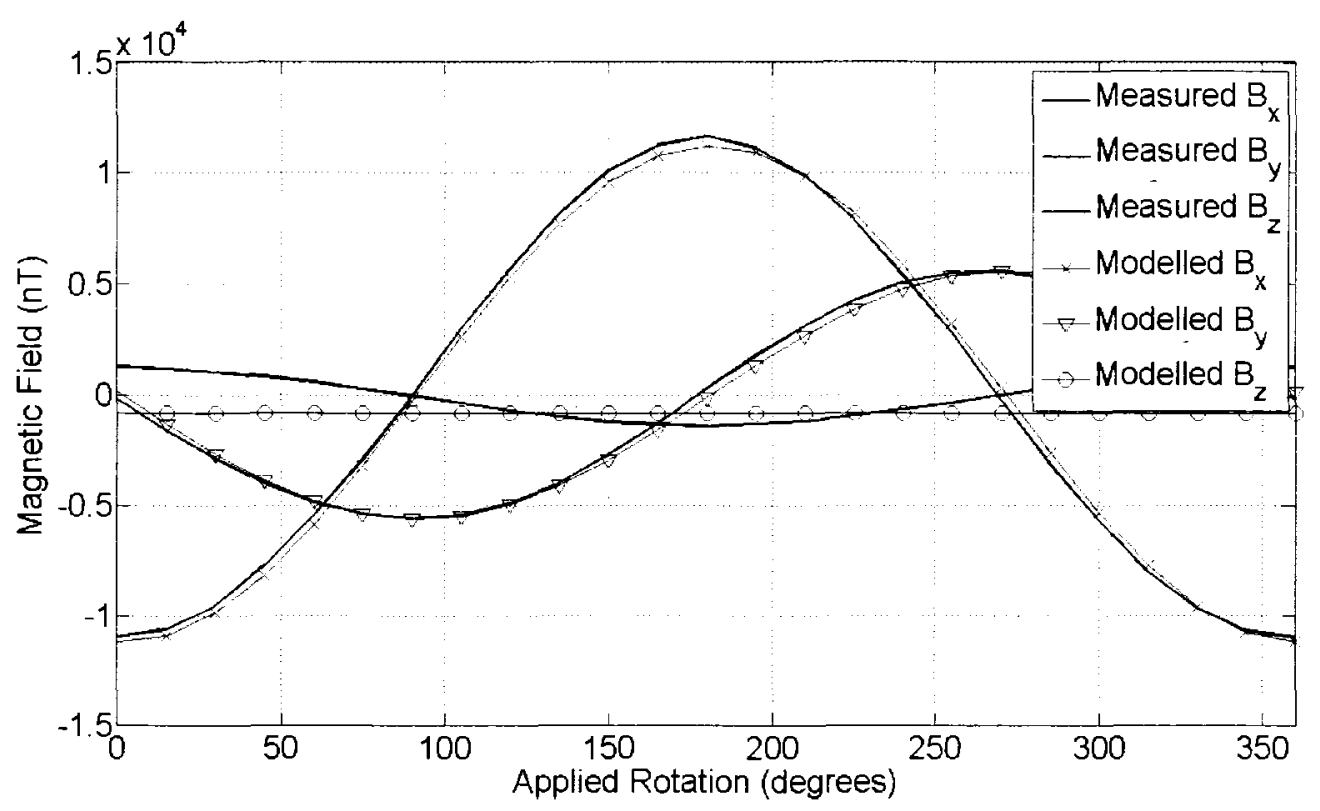

Figure 5.5: $\vec{B}$ vs. Applied Rotation: 2DP Direct Dipole Model. With the exception of the $B_{z}$ data the DDP/M estimates are in close agreement with the measured data.

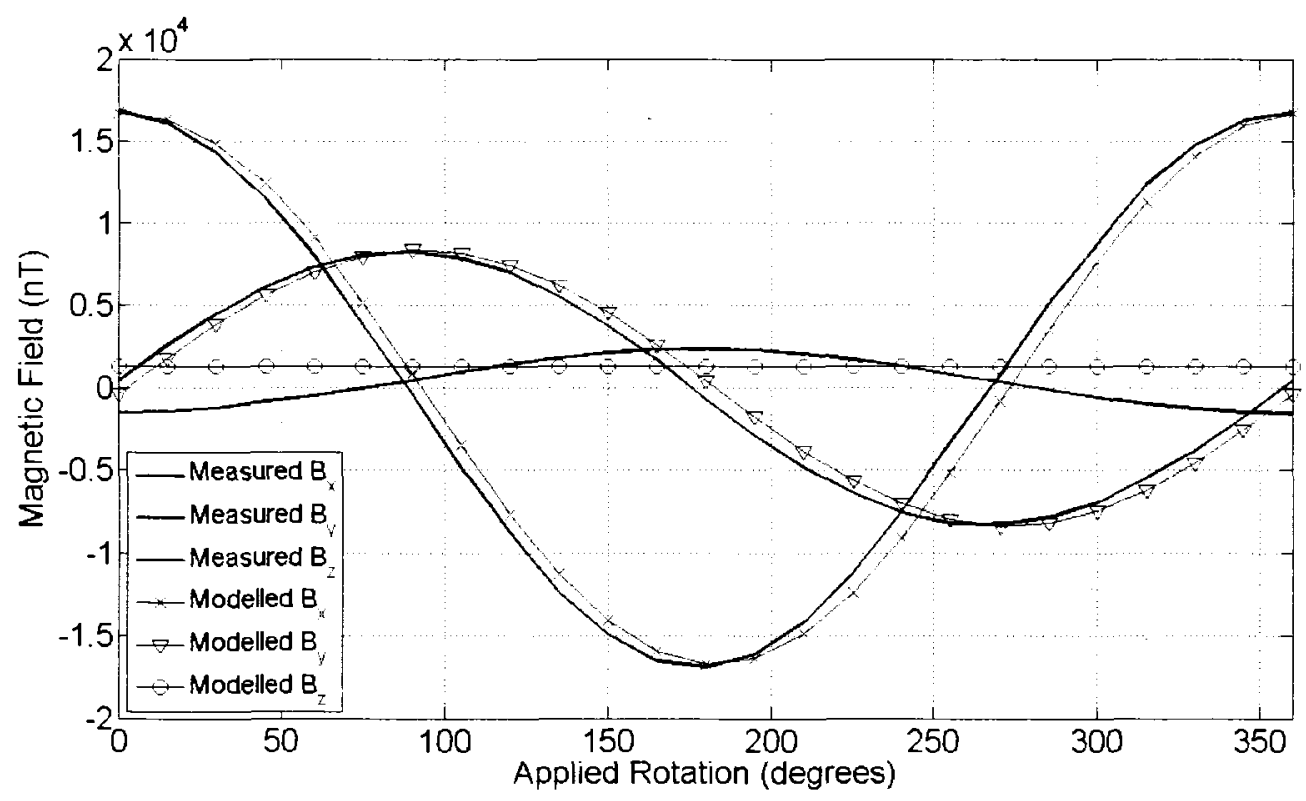

Figure 5.6: $\vec{B}$ vs. Applied Rotation: 3DPb Direct Dipole Model. With the exception of the $B_{z}$ data the DDP/M estimates are in close agreement with the measured data. The apparent phase shift notable in the $B_{x}$ and $B_{y}$ curves is attributed to errors in accurately rotating the source about its central axis. 


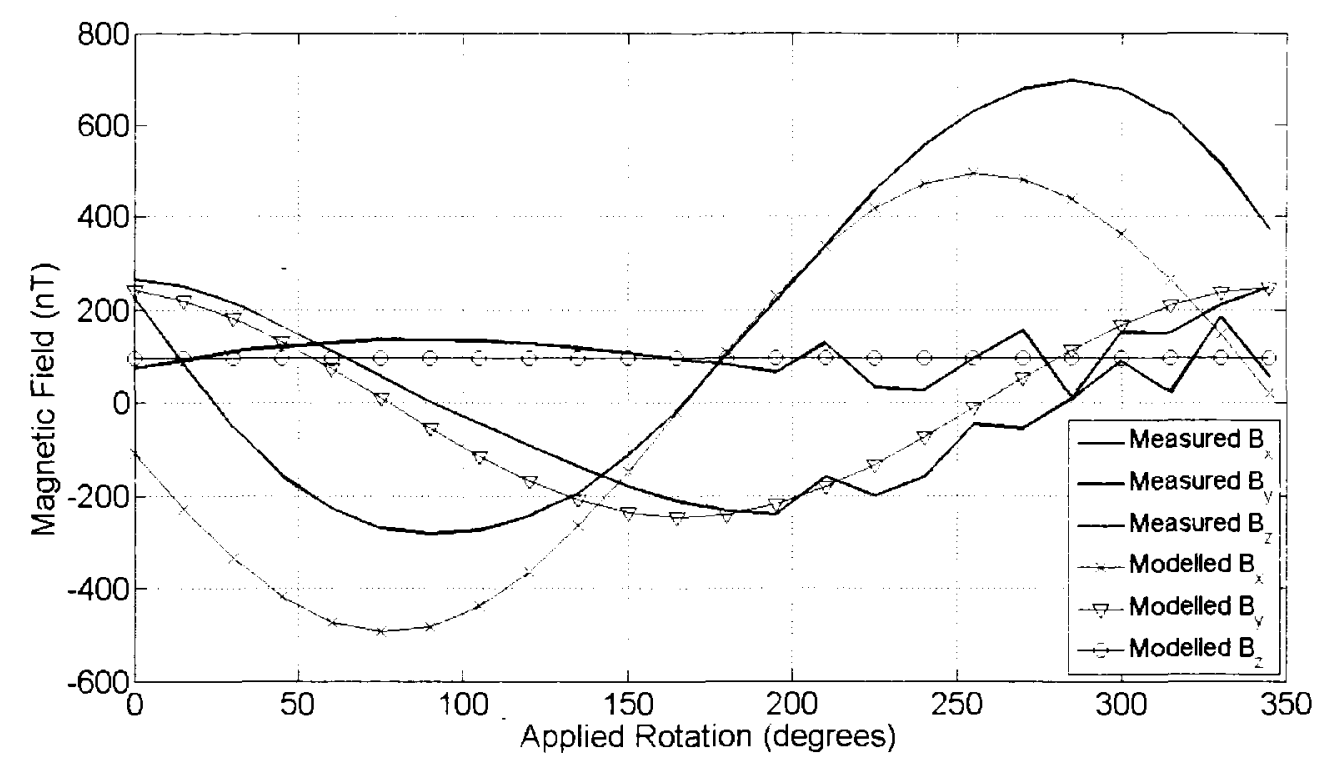

Figure 5.7: $\vec{B}$ vs. Applied Rotation: Futaba FP-S148 Direct Dipole Model. Poor fit between the modelled and measured curves is attributed to an offset between the axis of rotation and the location of the equivalent point-dipole. The sawtooth trend visible on the measured Bx and By curves reflect a functional resolution limitation; the background magnetic field became increasingly variable negating the estimate of background magnetic field for the $\mathrm{Y}$ and $\mathrm{Z}$ measurement channels.

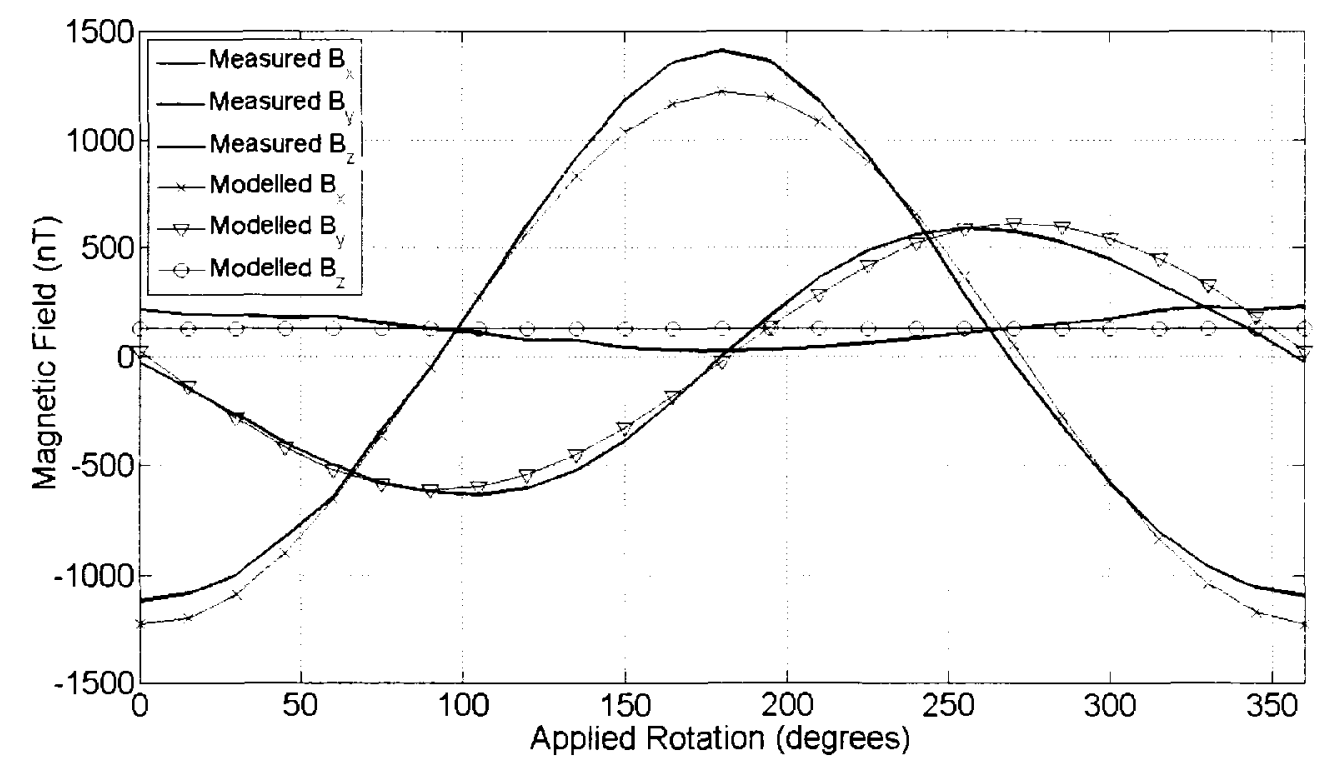

Figure 5.8: $\vec{B}$ vs. Applied Rotation: Hitec HSR-5995TG Direct Dipole Model. DDM/P estimates are in reasonable agreement with the measured values. 


\subsection{MoDEM Modelling}

The MoDEM model is a modification of the dipole ellipse model discussed in [9]. The purpose of the dipole ellipse was to represent polarized magnetic fields and consisted of two dipole vectors oriented with a $90^{\circ}$ phase difference between them. The MoDEM formulation is intended to add a certain amount of robustness to the modelling procedure in terms of applying the model for use in OFA.

\subsubsection{MoDEM Model Formulation}

The MoDEM model,

$$
\vec{B}=\left(\frac{\mu_{0}}{4 \pi}\right)\left\{\left[\frac{\left(3\left(\vec{m} 1 \cdot \hat{r}_{1}\right) \hat{r}_{1}-\vec{m} 1\right)}{\left\|\vec{r}_{1}\right\|^{3}}\right]+\left[\frac{\left(3\left(\vec{m} 2 \cdot \hat{r}_{2}\right) \hat{r}_{2}-\vec{m} 2\right)}{\left\|\vec{r}_{2}\right\|^{3}}\right]\right\}
$$

considers that the assigned coordinate system and axis of rotation may not be coincident with the point of action of the equivalent dipole. In this equation the magnetic field is calculated to the sum of contributions by two dipoles, $m 1$ and $m 2$.

Unlike in the dipole ellipse, the MoDEM model considers the two dipole moments as major (M1) and minor (M2); the magnitude of M2 is constrained to be less than M1. Rather than fixing the two at a $90^{\circ}$ offset, M2 may take any orientation relative to M1. Furthermore the effective point of action of the M2 vector may be shifted in the z-direction relative to $M 1$.

The offset between the assigned XYZ coordinate system of the MoDEM modelled source of interference and the location of the individual major and minor dipoles is 


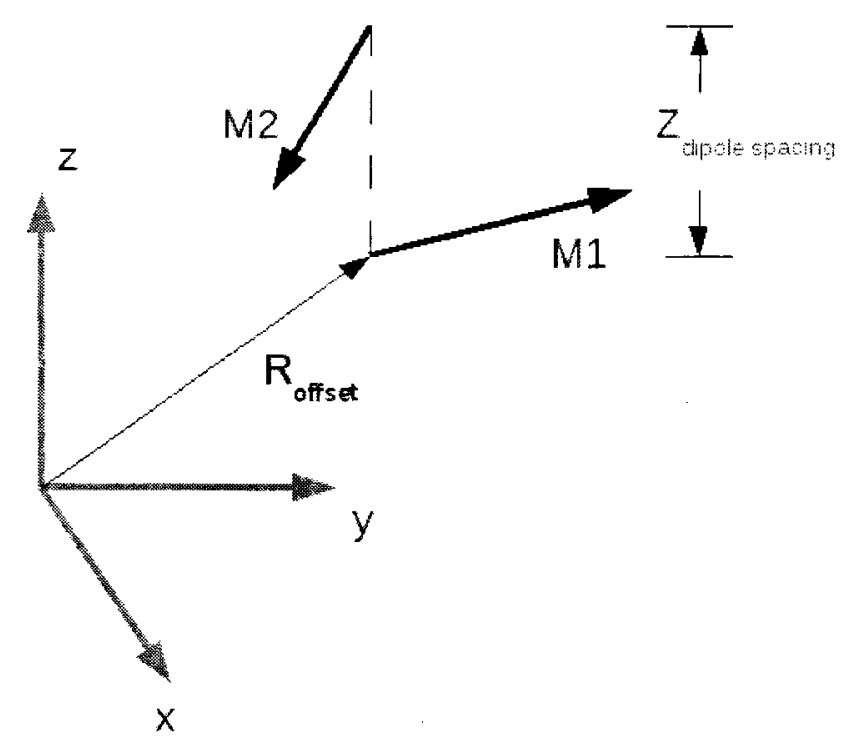

Figure 5.9: MoDEM Geometry. The major dipole (M1) is located at the origin of the model XYZ coordinate system whereas the minor dipole (M2) is offset along the $Z$-axis by some distance.

given by,

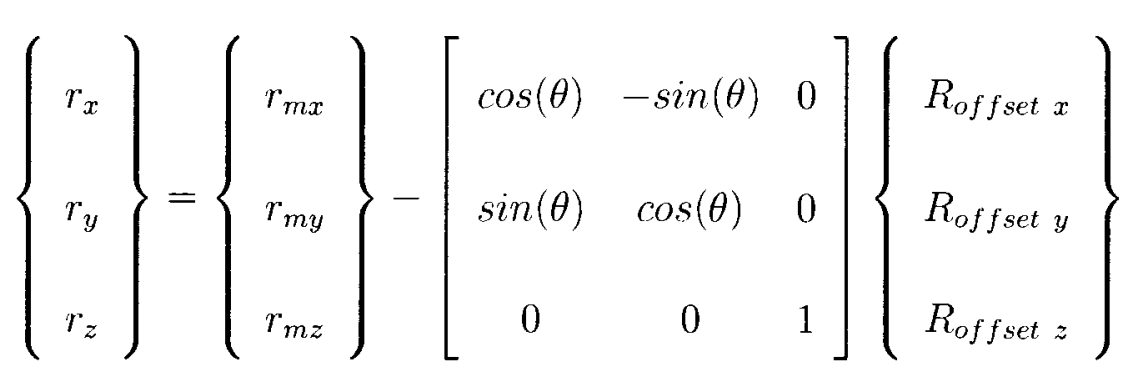

In the above equations, $\vec{r}$, describes the position of the measurement point from the assigned coordinate system of the source, $\vec{r}_{m}$ describes the offset vectors defining the position of major and minor dipoles with repsect to the source coordinate system, and $\theta$ is the applied rotation of the source about the assigned source coordinate system. These variables are described graphically in Figure 5.9.

The added complexity inherent in the MoDEM approach was deemed necessary for the following reasons: 
1. The magnetic centroid, defined as the location of the equivalent dipole moment, is rarely located at a convenient location for actuating system components. Furthermore, determining the effective location can be difficult. Rotating about a point other than the magnetic centroid will result in the equivalent dipole undergoing a combination of rotation and translation. The resulting measurements will reveal non-dipolar characteristics that cannot be reproduced by the direct dipole modelling strategy.

2. An ideal dipole rotating perfectly in the same plane as a point of observation will produce a magnetic field witl no z-component. Furthermore, the same ideal dipole oriented out-of-plane and rotating about the sanne point will generate a z-component that does not change with respect to rotation. Adding a second dipole moment vector to the model should add the additional degrees of freedom necessary to capture the inevitable variability in the z-direction measurements.

3. Many actuating system components, such as servos, consist of multiple individual magnetic elements. DC motors, for example, may include several dipole magnets in the stator. Although dipole equivalence modelling assumes that, given sufficient separation between source and observation point, the magnetic field becomes dipolar in the far field region [9], the hypothesis is that by adding an additional moment vector some of the variability in the near-field region may be captured and the region of model applicability increased.

\subsubsection{MoDEM Parameterization}

There are ten parameters $\left(\mathrm{M}_{x, y, z}, \mathrm{M} 2_{x, y, z}, \mathrm{R}_{\text {Offset } x, y, z}, \mathrm{Z}_{\text {Dipole Spacing }}\right)$ in the MoDEM equation; it is not possible to determine these variables analytically. A nonlinear quadratic programming-based function minimization algorithm (Matlab fmincon) 
was used to determine an optimal estimate for the ten parameters. A package of Matlab scripts, MoDEMmaster.m, MoDEMobjective.m, MoDEMtest.m, and MoDEMconstraint.m, were developed to perform the MoDEM parameterization; these scripts are located in Appendix C.

\section{Objective}

The MoDEM parameterization objective function,

$$
\text { Error }=\frac{\sum_{1}^{N}\left(\left\|\vec{B}_{\text {measure }}\right\|-\left\|\vec{B}_{M o D E M}\right\|\right)^{2}}{N}
$$

considers the mean square error between the modelled and measured magnetic field magnitude $\|\vec{B}\|$. The optimization algoritlım seeks to minimize this error. By considering the magnitude of the ficld, equal weighting is given to fitting the MoDEM model to the individual $x, y$, and $z$ curves.

\section{Constraints}

The MoDEM parameterization algorithm uses inequality and boundary constraints. The inequality constraint,

$$
0 \leq\|\vec{M} 1\|-\|\vec{M} 2\|
$$

ensures that the major dipole monent vector is larger than the minor. Bounding constraints are considered for all 10 model parameters but the values are considered arbitrary. It makes sense to ensure that the offset vector and the dipole spacing vector do not place either dipole moment vector outside the physical dimensions of the device. For each of the five objects that were tested the following upper and lower bounding constraints were considered:

- $\mathrm{M}_{x, y, z}$ and $\mathrm{M} 2_{x, y, z}: \pm 10^{9} \mathrm{Am}^{2}$ 


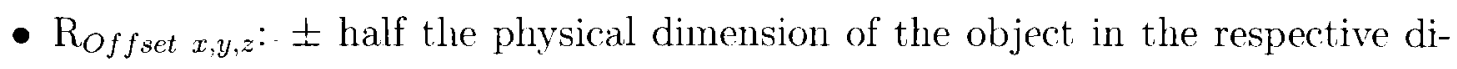
mensions.

- 2DP: $\mathrm{R}_{\text {Offset } x, y, z}=\left[\begin{array}{lll}0.01 & 0.01 & 0.01\end{array}\right] \mathrm{m}$

- 3DP(a and $\mathrm{b}): \mathrm{R}_{\text {Offset } x, y, z}=[0.0150 .010 .01] \mathrm{m}$

- Futaba FP-S148: Roffset $x, y, z=[0.020 .0150 .01] \mathrm{m}$

- Hitec HSR-5995TG: R of fset $x, y, z=\left[\begin{array}{lll}0.02 & 0.015 & 0.09\end{array}\right] \mathrm{m}$

- $\mathrm{Z}_{M 1 M 2 \text { Spacing }} \pm 0.01 \mathrm{~m}$

\subsubsection{MoDEM Test Results}

Table 5.2 lists the absolute mean error for the MoDEM algorithm validation testing. Figures 5.10 to 5.11 plot the MoDEM modelled vs. experimentally neasured $\vec{B}$ vs. applied rotation for the bar magnet sources. Figures 5.12 to 5.13 plot the MoDEM modelled vs. experimentally measured $\vec{B}$ vs. applied rotation for the two servo actuators. A direct comparison between the DDP/M and MoDEM algorithms will be presented in the next section.

With the exception of the Futaba servo results, Figure 5.12. the MoDEM algorithm provided an excellent match between the predicted and measured magnetic fields. The results for the Futaba servo were mixed. Excellent agreement between the $B_{x}$ curves was noted, however the variation in the $B_{y}$ and $B_{z}$ data likely affected the ability of the MoDEM parameterization algorithm to accurately match those curves. 
Table 5.2: Mean Absolute Modelling Error (nT) for the MoDEM Field Estimation Algorithm

\begin{tabular}{|c|c|c|c|c|}
\hline Source & $B_{x}$ & $B_{y}$ & $B_{z}$ & $\|\vec{B}\|$ \\
\hline \hline $2 \mathrm{DP}$ & 98.8 & 156.0 & 829.5 & 62.4 \\
\hline $3 \mathrm{DPa}$ & 157.6 & 219.2 & 1218.7 & 105.1 \\
\hline $3 \mathrm{DPb}$ & 125.4 & 197.4 & 1259.7 & 103.9 \\
\hline Futaba & 11.41 & 28.2 & 47.9 & 5.13 \\
\hline Hitec HSR-5995TG & 14.93 & 22.5 & 51.7 & 10.15 \\
\hline
\end{tabular}

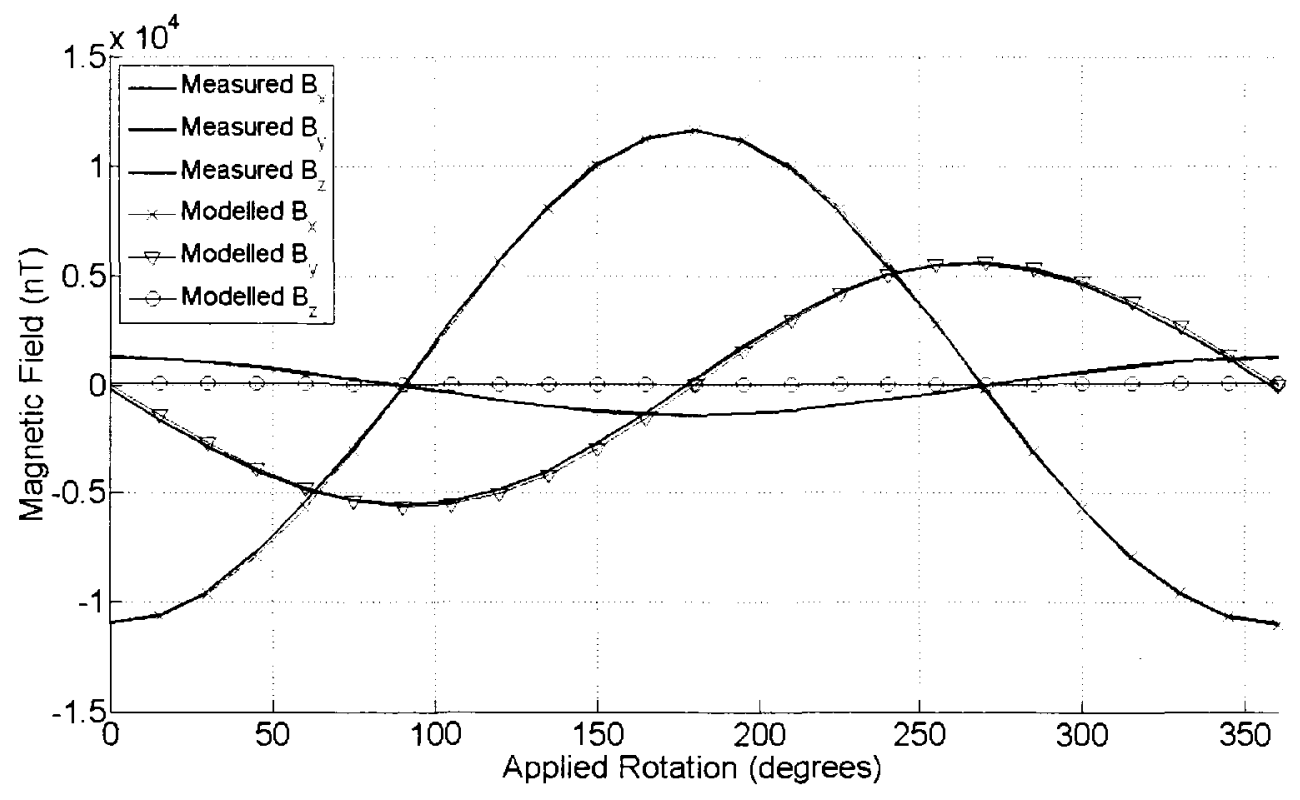

Figure 5.10: $\vec{B}$ vs. Applied Rotation: 2DP MoDEM. Excellent match between the measured and MoDEM modelled curves is observed. 


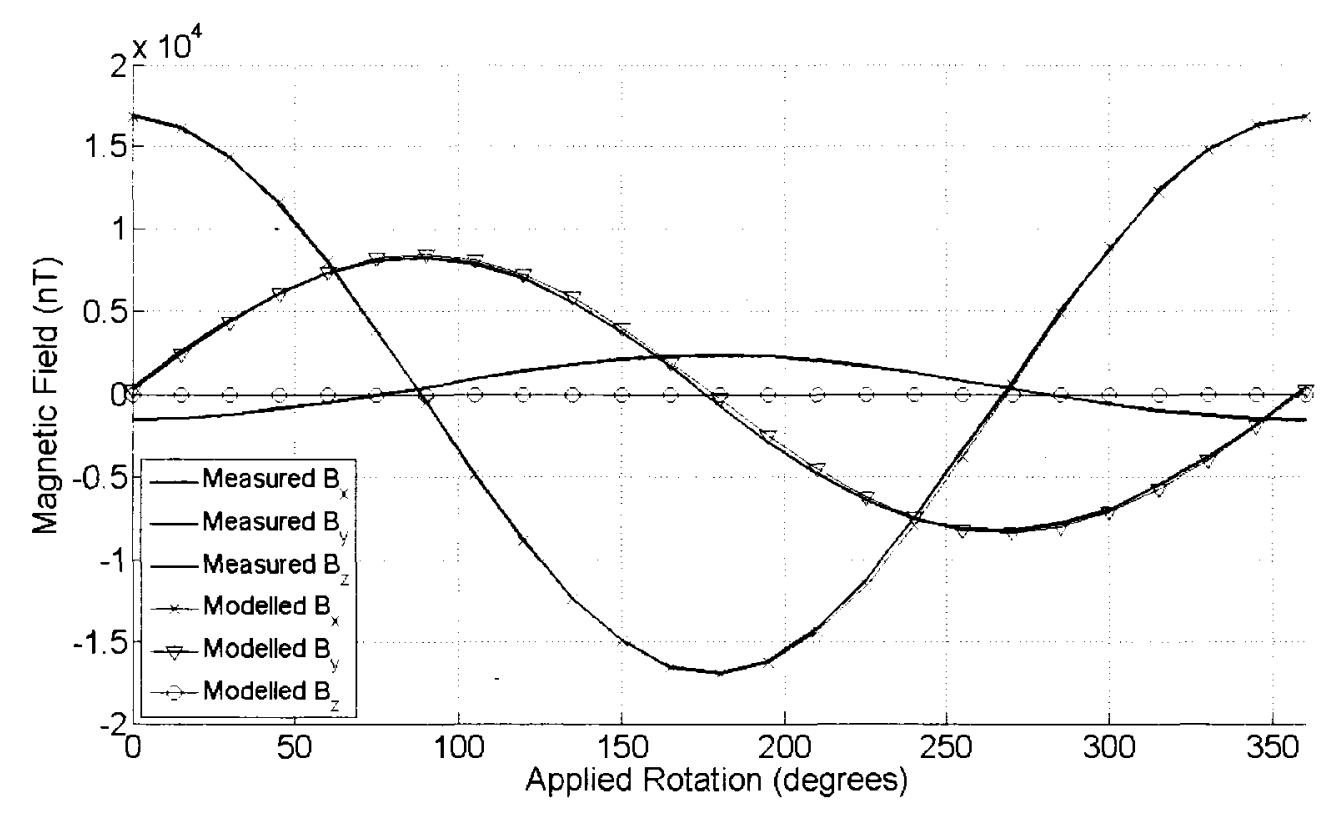

Figure 5.11: $\vec{B}$ vs. Applied Rotation: 3DPb MoDEM. Excellent match between the measured and MoDEM modelled curves is observed.

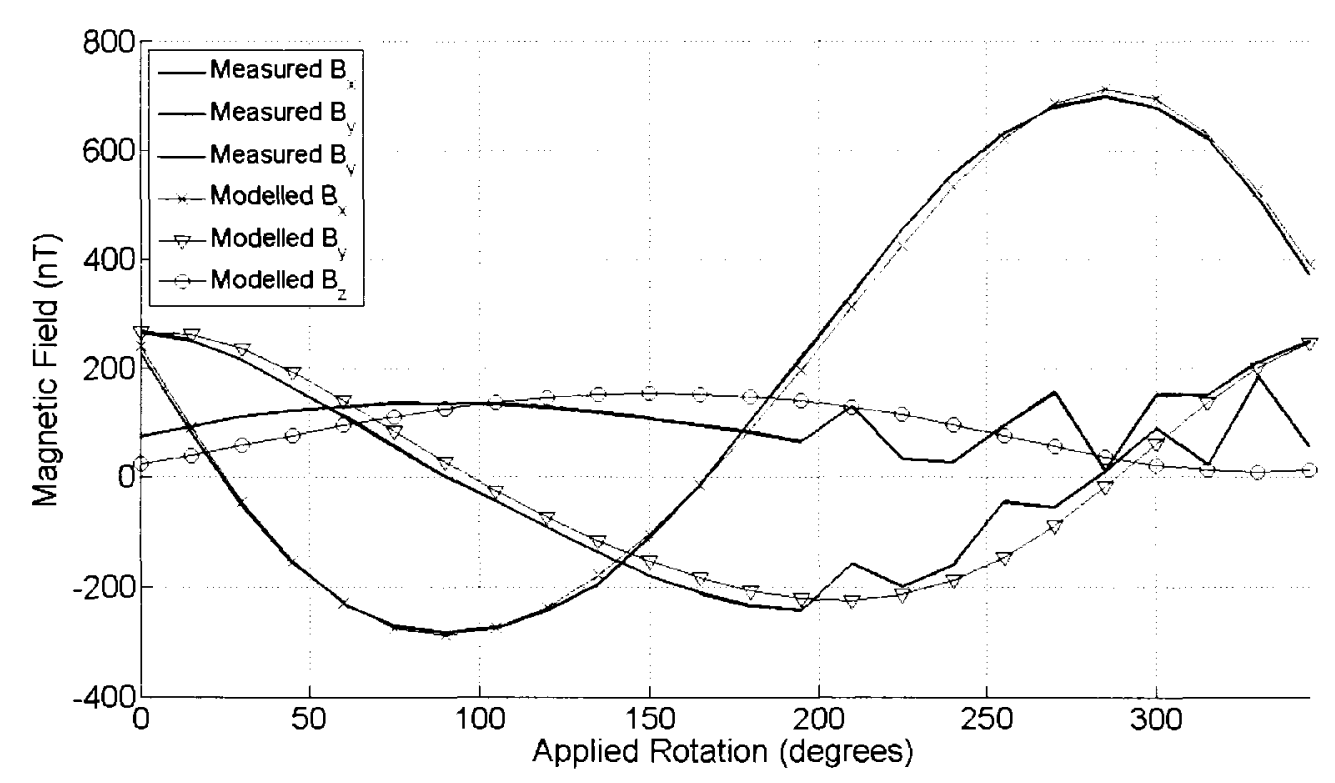

Figure 5.12: $\vec{B}$ vs. Applied Rotation: Futaba FP-S148 MoDEM. Good fit between the measured and MoDEM modelled curves is observed. Error in the $B_{y}$ and $B_{z}$ curves is attributed to the variability in the measured data. 


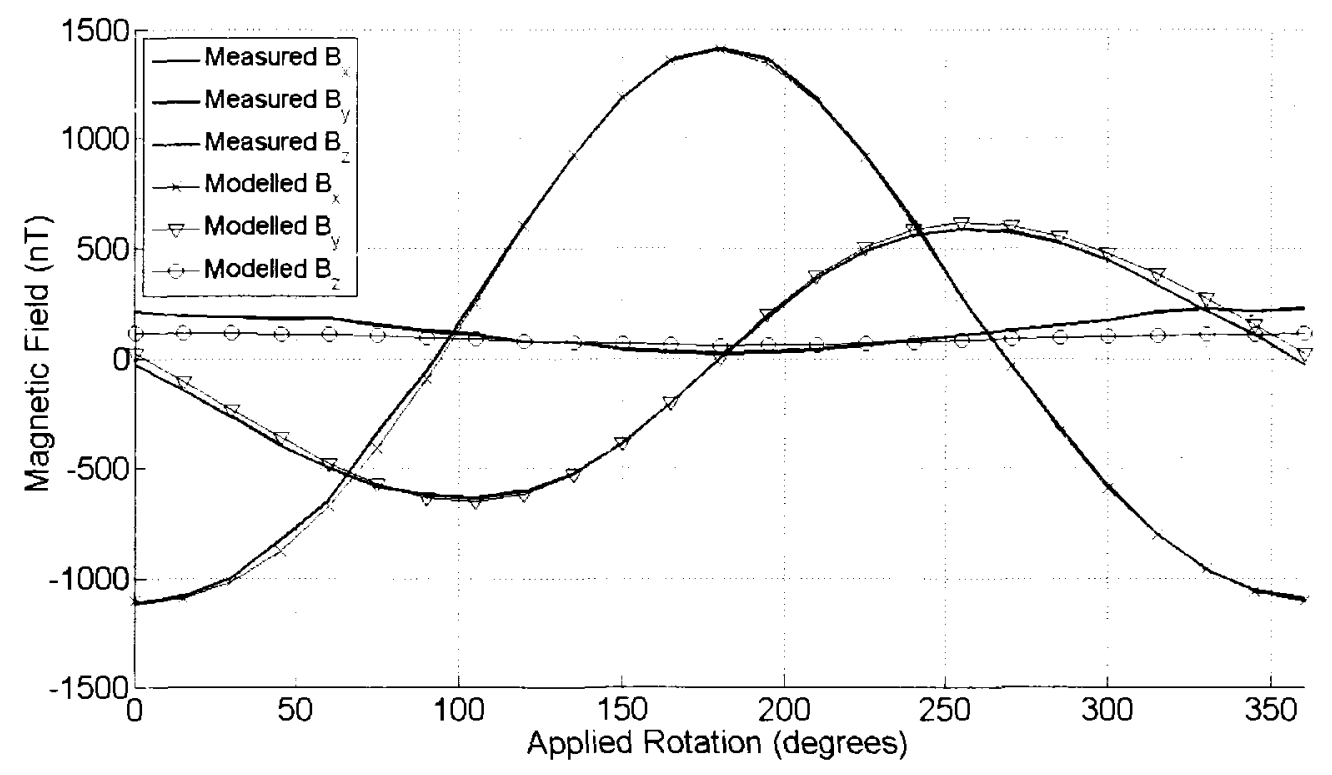

Figure 5.13: $\vec{B}$ vs. Applied Rotation: Hitec HSR-5995TG MoDEM. Excellent match between the measured and MoDEM modelled curves is observed.

\subsection{Model Selection}

Figures 5.14 to 5.17 compare the direct dipole and MoDEM estimates of $\|\vec{B}\|$ with the measured data. For the five modelled field sources, the difference between the MoDEM and DDP/M data is listed in Table 5.3. This is interpreted as the MoDEM improvement over the DDM/P algorithm.

Table 5.3: MoDEM improvement over DDP (nT)

\begin{tabular}{|c|c|c|c|c|c|}
\hline & $2 \mathrm{DP}$ & $3 \mathrm{DPa}$ & $3 \mathrm{DPb}$ & FP-S148 & HSR-5995TG \\
\hline \hline $\mathrm{x}$ & 243.96 & 304.62 & 787.81 & 620.66 & 63.22 \\
\hline $\mathrm{y}$ & 90.66 & 148.56 & 409.94 & 274.34 & 26.29 \\
\hline $\mathrm{z}$ & 203.14 & 172.1 & 149.96 & -12.54 & 9.41 \\
\hline $\mathrm{mag}$ & 145.81 & 121.4 & 340.55 & 131.82 & 66.65 \\
\hline
\end{tabular}

For all field sources, using both the DDP and MoDEM modelling algorithms, the $\mathrm{B}_{z}$ field component was poorly modelled on a consistent basis. With the exception 
of the Futaba FP-S148, the MoDEM algorithm provides a better match to $\mathrm{B}_{z}$ than did DDP, although the improvements were minor. For all other components of the field the MoDEM algorithm proved superior to DDP. When comparing the $\|\vec{B}\|$ modelling error presented in Tables 5.1 and 5.2, for the DDM and MoDEM algorithms respectively, the error was calculated as,

$$
\left\|\vec{B}_{\text {error }}\right\|=\sum_{1}^{N} \frac{\left\|\vec{B}_{\text {model } n}\right\|-\left\|\vec{B}_{\text {measure } n}\right\| \mid}{N}
$$

where $\mathrm{N}$ corresponds to the number of samples. This is the same error definition that was used for the field component errors. Defining the $\|\vec{B}\|$ modelling error as,

$$
\left\|\vec{B}_{\text {error }}\right\|=\sqrt{B_{x \text { error }}^{2}+B_{z \text { error }}^{2}+B_{z \text { error }}^{2}}
$$

would result in an over-estimate of the actual magnitude error.

Figures 5.14 to 5.17 show that the modelling results for the MoDEM algorithm are superior to the DDP algorithm. The DDP algorithm assumes that the equivalent dipole undergoes pure rotation and that the sensor is situated and oriented in the same plane as the field source. This results in modelled field characteristics that are constrained to be ideally dipolar. The MoDEM algorithm is not affected by these rigid constraints. By considering the equivalent point dipole location and the source coordinate system to be separated allows for the modelling of non-ideally dipolar fields. This characteristic is most clearly illustrated by Figure 5.16. For these reasons the MoDEM model was selected for use as part of the OFA algorithm discussed in the next chapter.

Figures 5.18 and 5.19 plot the error between the measured vs. modelled data for the 3DP and Hitec servo collected as a line profile. Measurements were taken starting 
at $0.1 \mathrm{~m}$ from the sensor and then moving along the $\mathrm{x}$-axis at increments of 0.02 $m$. These indicate that as the separation between the field source and observation (measurement) point increase the model accuracy increases as well.

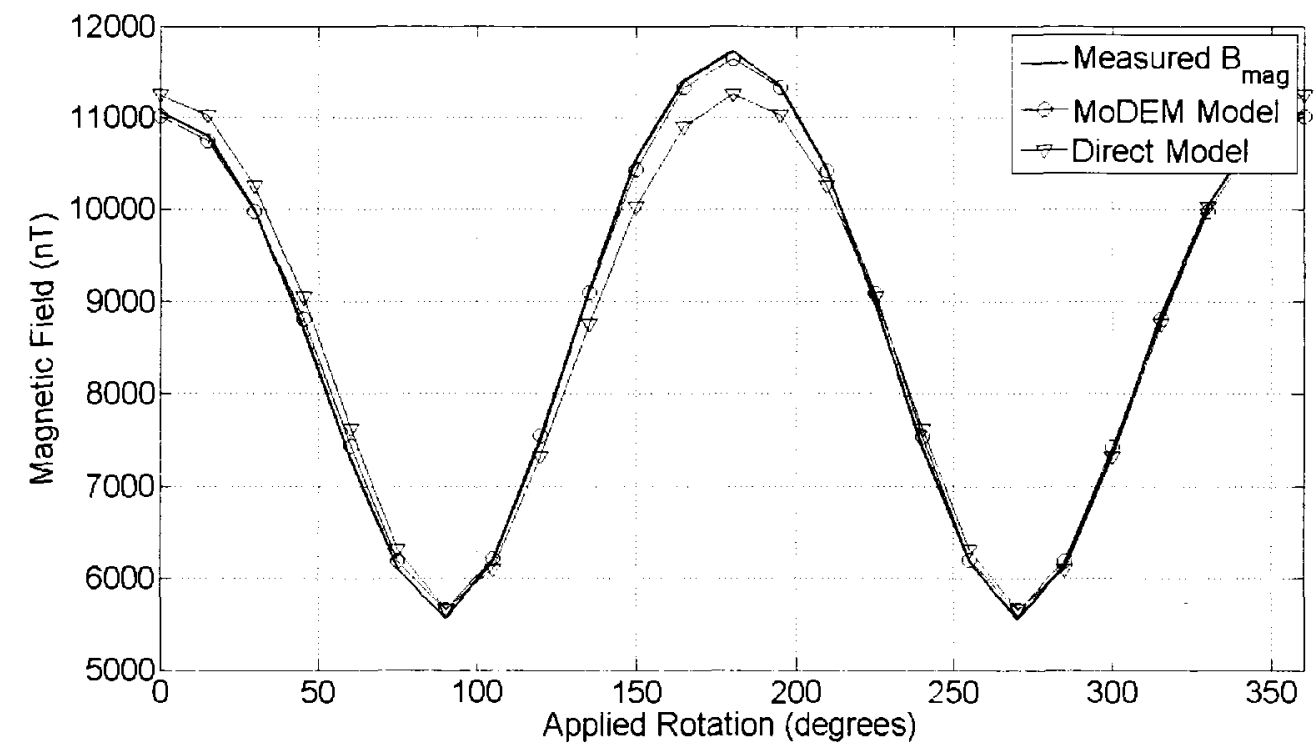

Figure 5.14: Comparison Between MoDEM and Direct Dipole Model Estimates: 2DP 


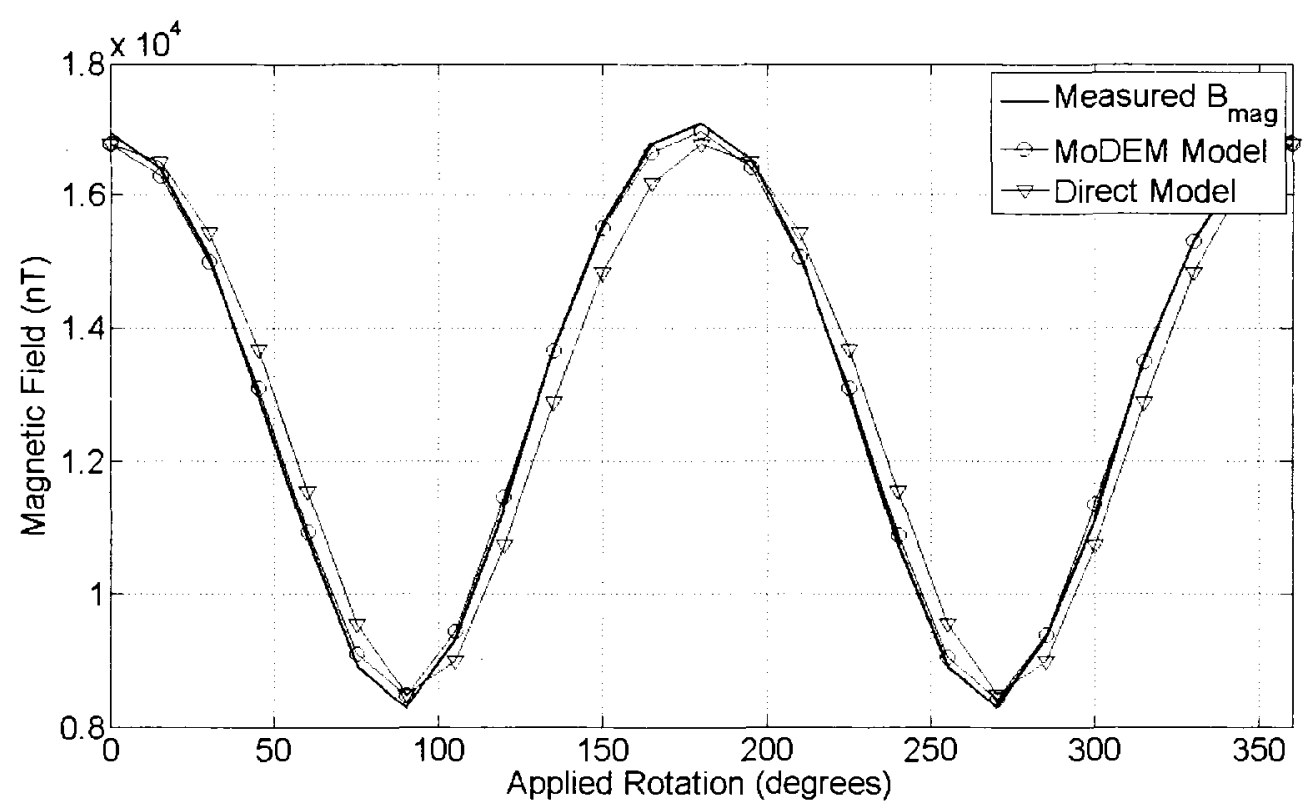

Figure 5.15: Comparison Between MoDEM and Direct Dipole Model Estimates: $3 \mathrm{DPb}$

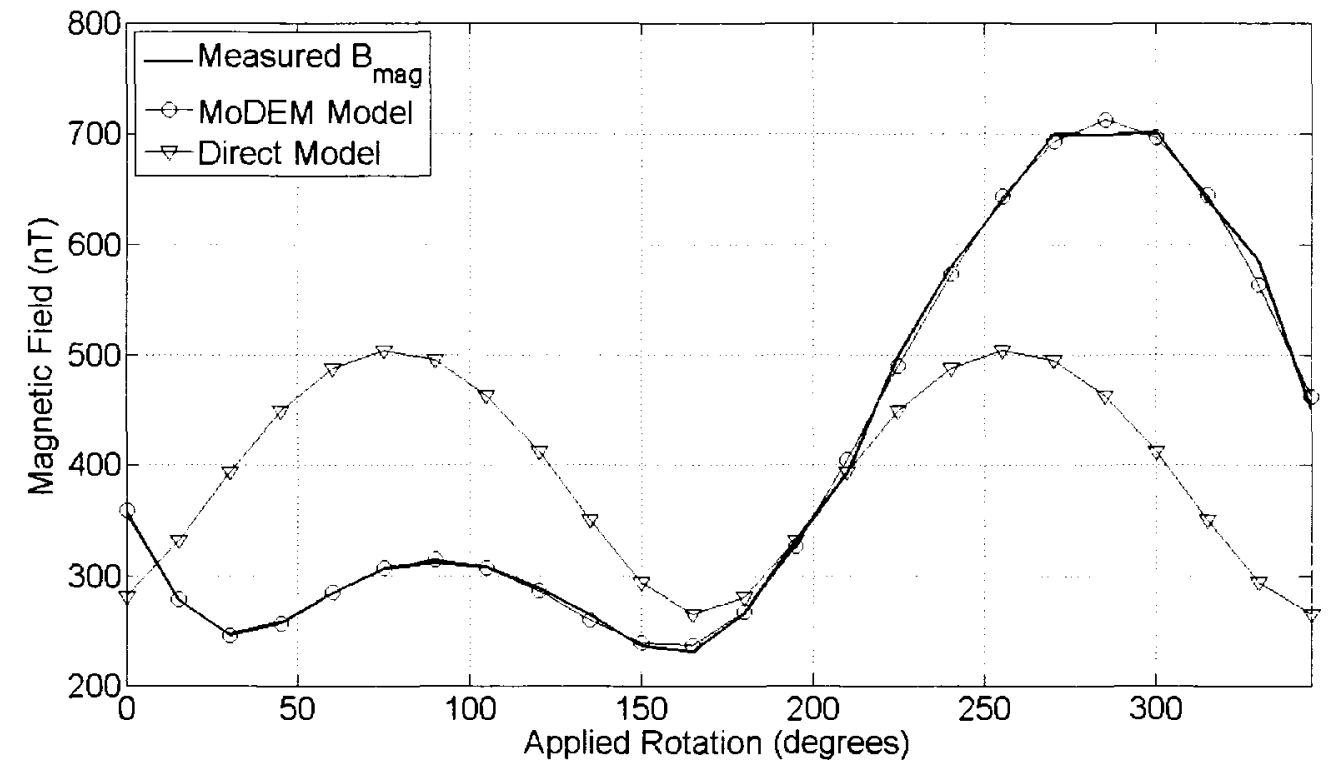

Figure 5.16: Comparison Between MoDEM and Direct Dipole Model Estimates: Futaba FP-S148 


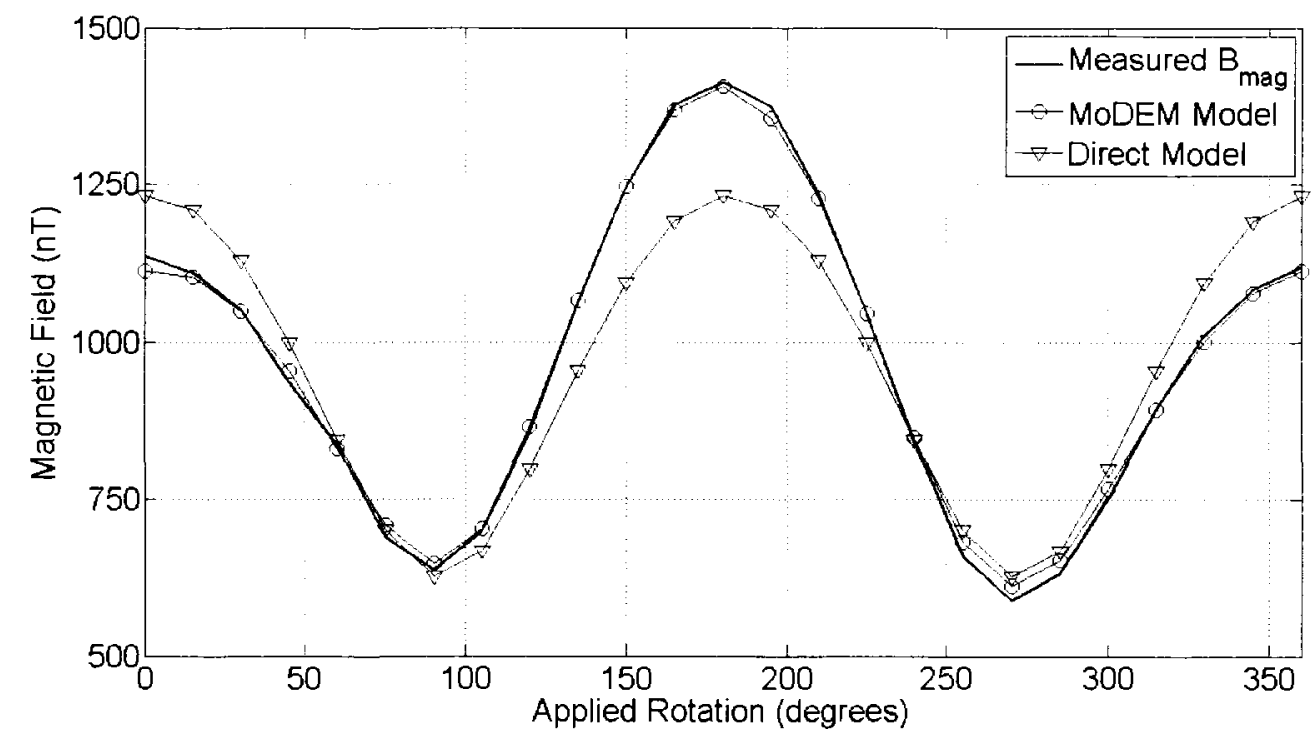

Figure 5.17: Comparison Between MoDEM and Direct Dipole Model Estimates: Hitec HSR-5995TG

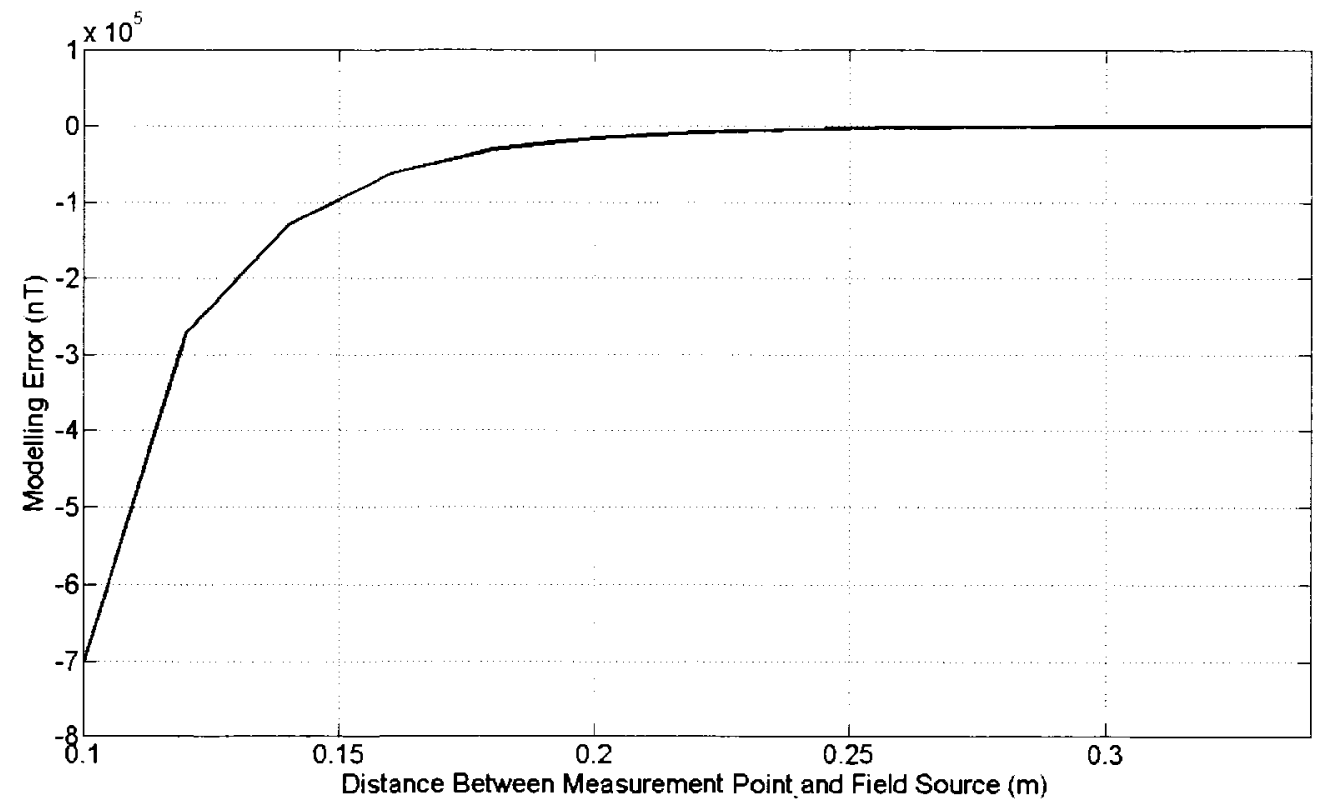

Figure 5.18: MoDEM Modelling Error - 3DP Source. The error decays to zero as the measurement distance increases. 


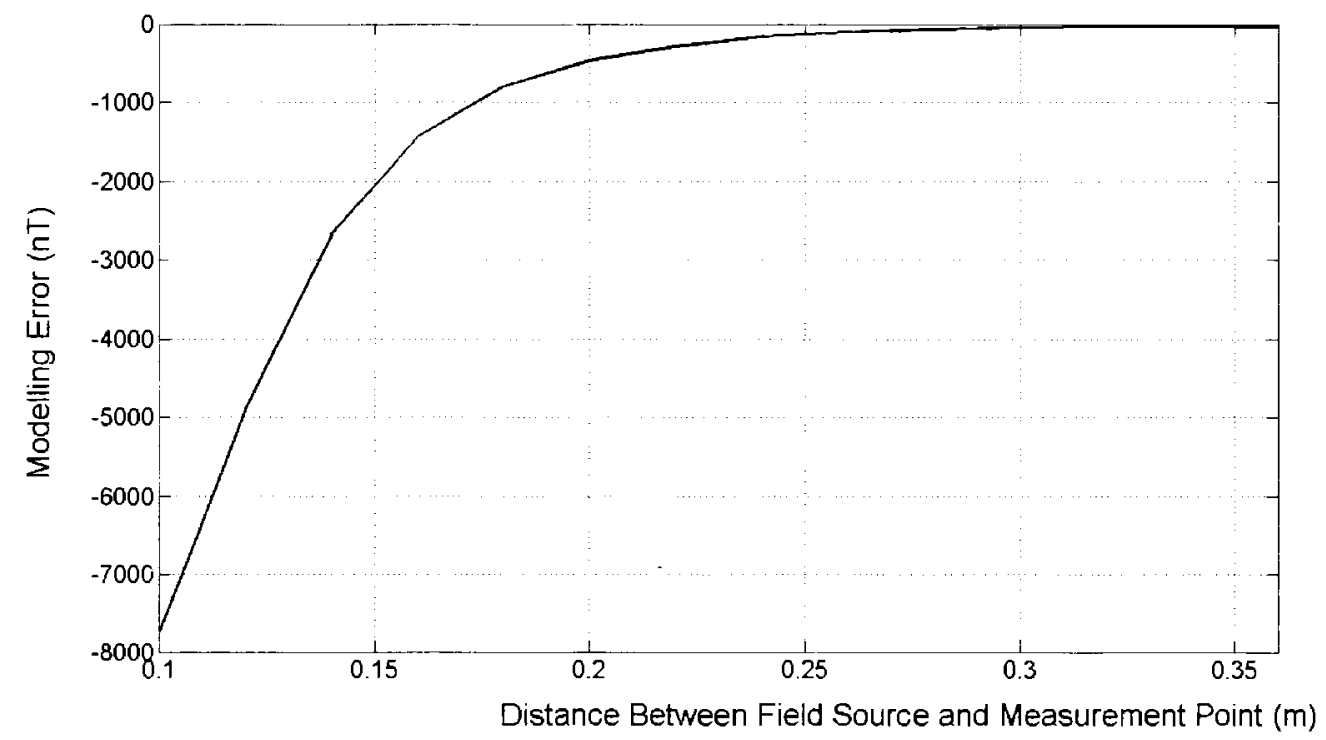

Figure 5.19: MoDEM Modelling Error - Hitec HSR-5995TG. The error decays to zero as the measurement distance increases. 


\section{Chapter 6}

\section{Optimal Field Attenuation}

\subsection{Introduction}

Optimal Field Attenuation (OFA) is a novel attenuation strategy for minimizing,

and/or spatially shaping, magnetic fields over desired regions of interest. Although the algorithm was developed for the GeoSurv II aircraft, it is suitable for attenuating interference in any system that is sensitive to time-invariant magnetic fields.

The core OFA algorithm seeks to configure sources of magnetic interference such that their combined magnetic fields are minimized and spatially stabilized in the area(s) of desired attenuation. The set of possible positions and orientations for the sources of interference is referred to as the configuration envelope for the system. Two design cases for implementing OFA were identified:

1. Parallel Design: By considering the OFA algorithm during the design stage, the maximum possible configuration envelope can be used to achieve inaximum interference attenuation. GeoSurv II falls under this category.

2. Re-Design: Implementing OFA in systems that are currently in operation, or in systems with very small configuration envelopes (heavily constrained), is 
possible by applying the algorithm to a set of magnetic sources that can be added to the system.

This chapter will present the OFA algorithm and discuss the theoretical implementation of the algorithm for GeoSurv II. The field measurement system is not suitable for experimentally verifying the GeoSurv II implementation; experimental validation of the strategy will be shown using a three-source configuration of MoDEM modelled field sources. This section will also present the theoretical results of applying OFA on GeoSurv II using a configuration of five Hitec servos; one for each control surface. An investigation into the relationship between optimization area and achievable attenuation will also be discussed.

\subsection{OFA Background}

The three principal objectives of the OFA algorithm are to attenuate, spatially stabilize, and equalize the average time-invariant magnetic interference acting over magnetically sensitive components in a system. Here attenuation refers to the reduction in the average magnetic field by the OFA algorithm. Spatial stability refers to minimizing the variation in the magnetic field over the optimization area. The final objective, equalization, is considered for multi-area optimization problems such as the two magnetometers on GeoSurv II. The OFA algorithm must equalize the average magnetic field at each magnetometer. The following section describes the background rationale for the decisions and objectives considered in the development of the algorithm. 


\subsubsection{Time Variant vs. Time Invariant}

Design targets of $0.1 \mathrm{nT}$ for time-invariant, and $0.01 \mathrm{nT}$ for time-variant interference are considered on GeoSurv II. Magnetic testing of servo actuators was conducted for devices in both a power-on dynamic position and power-off static position series of configurations $[35,36]$. It was concluded that the difference in magnetic interference measured between the two cases was not significant, indicating that the time invariant interference was dominant. Accordingly, OFA extensions to time-variant interference were not considered in this thesis.

\subsubsection{Optimal Attenuation}

The relationship between the size of the optimization region and the spatial stability of the field will be described in detail in Section 6.5.1. Initially, when the OFA algorithm was proposed, the strategy considered point optimization; the objective function considered only the magnitude of the field at a discrete point in space. A consequence of optimizing for a point is that the field will be minimized at the optimal point, large field gradients will result in large measurements in the vicinity. This is not desirable; in a real application the location of a sensor with respect to the sources of magnetic interference may be variable. To alleviate this issue, the second objective for OFA, spatial field stability, was developed.

\subsubsection{Spatial Field Stability}

For many magnetometers it is difficult to determine a discrete location where the field sensing occurs. The Honeywell HMR-2300 magnetometer used in the magnetic measurement system developed for this thesis includes two magnetoresistive sensing integrated circuits in a configuration shown in Figure 6.1. The in-plane (XY) chip measures approximately $0.015 \mathrm{~m} \mathrm{x} 0.01 \mathrm{~m}$, the out-of-plane $(\mathrm{Z})$ field chip measures 
approximately $0.01 \mathrm{~m} \mathrm{x} 0.005 \mathrm{~m}$. If an assumption is made that the sensor measures the average field over these areas then it can be argued that the average field in the vicinity of the sensing element should be considered when solving for the optimal system configuration.
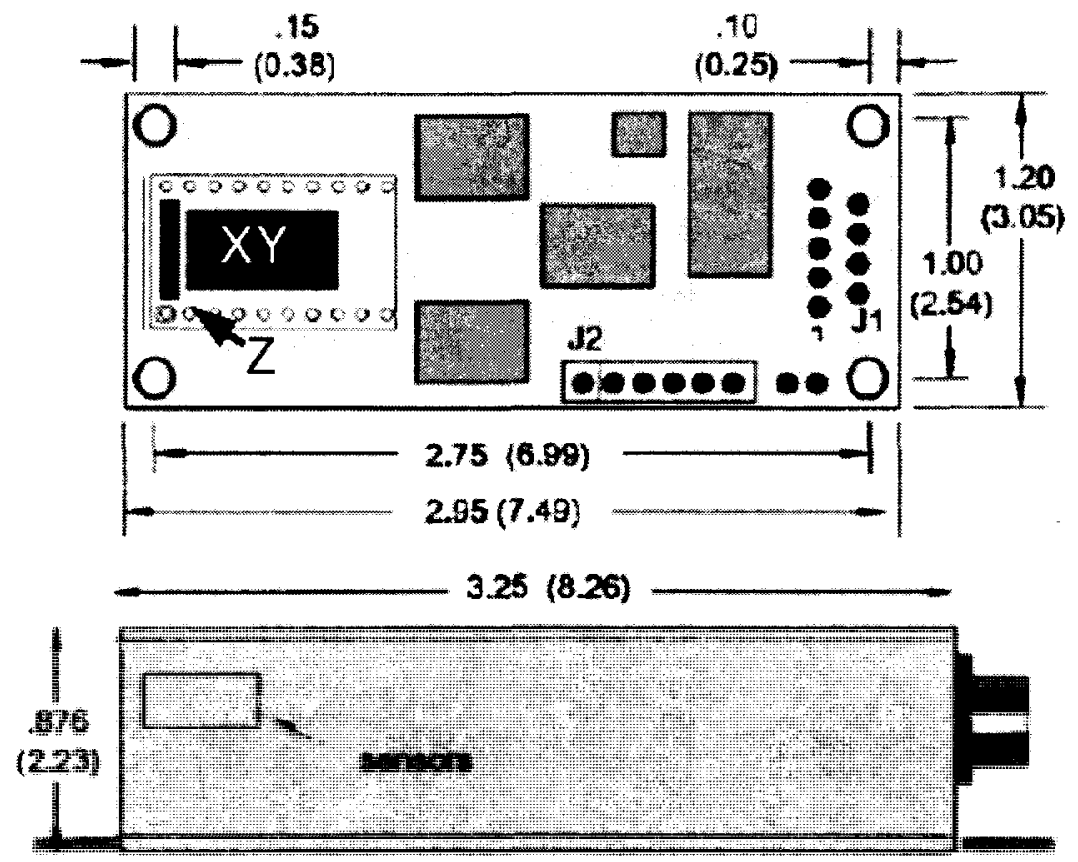

Figure 6.1: HMR-2300 Circuit Schematic. The HMR-2300 magnetometer uses the Honeywell HMC 2003 3-axis magnetoresistive magnetometer integrated circuit.

The optimization problem, with an objective of minimizing the average field over an area surrounding a sensitive component, yields a flatter distribution of magnetic field intensity. Spatial field stability refers to the rate at which the field increases as we move further from the optimum point; it is a measure of the sensitivity of the field to positional variations. The GeoSurv II UAV will be equipped with highly sensitive optically pumped-alkali magnetometers mounted on both wingtips. Spatial field stability is considered critical for the GeoSurv II application. 


\subsubsection{Field Equalization}

GeoSurv II will include magnetometers on both wingtips in order to measure the field gradient across the wingspan (gradiometer). In addition to minimizing and spatially stabilizing the magnetic field at the two magnetometers, it is critical to equalize the magnetic field at each. The requirements for magnetic interference are defined for the absolute interference affecting an individual magnetometer. In a gradiometer configuration, such as the one used on GeoSurv II, there are two sensors which are used to measure the magnetic field gradient between them. If the magnetic interference is equalized at both sensors, then measuring the gradient of the field should result in the interference being eliminated.

\subsection{Optimal Field Attenuation Algorithm}

The OFA configuration solver uses the same Matlab constrained function minimization algorithm, fmincon, that was discussed in Section 5.4. The following section will present the objective function, and constraints considered for single and multiple objective cases. The Matlab OFA script package is included in Appendix D.

\subsubsection{Objective}

The objective of OFA is to minimize the average magnetic field in the area surrounding a sensitive system component. The objective function for a single area OFA analysis is,

$$
B_{o p t}=\min \sum_{1}^{W}\left[\frac{\left\|\sum_{1}^{N} \vec{B}_{n, w}\right\|}{W}\right]
$$

where $B_{o p t}$ refers to the optimized field. $\vec{B}_{n, w}$ corresponds to the field produced by interference source, $\mathrm{n}$, measured at point, $\mathrm{w}$, within the area of optimization. To account for multiple areas of optimization the objective function must be modified. 
For a two area, A1 and A2, optimization problem, the objective function is given by,

$$
B_{\text {optimum }}=\min \left[B_{A 1+A 2}+a b s\left(K B_{A 1-A 2}\right)\right]
$$

where $B_{A 1}$ and $B_{A 2}$ refer to, the average magnetic field magnitudes calculated over the two optimization areas in according to Equation 6.1 and $\mathrm{K}$ is a penalty term. Therefore,

$$
B_{A 1+A 2}=\sum_{1}^{W}\left[\frac{\left\|\sum_{1}^{N} \vec{B}_{A 1 n, w}+\sum_{1}^{N} \vec{B}_{A 2 n, w}\right\|}{W}\right]
$$

and

$$
B_{A 1-A 2}=\sum_{1}^{W}\left[\frac{\left\|\sum_{1}^{N} \vec{B}_{A 1 n, w}-\sum_{1}^{N} \vec{B}_{A 2 n, w}\right\|}{W}\right]
$$

respectively.

The OFA objective is to minimize the interference field averaged over an area of interest; in the case of GeoSurv II, which uses two magnetometers, two areas must be considered (A1 and A2). Using MoDEM models of the sources of interference, the solver seeks to minimize the combined effects of $\mathrm{N}$-sources of interference averaged over an area sampled by $\mathrm{W}$ measurements.

Equation 6.1 describes the objective function for a single area OFA case. When the objective is to minimize the field over more than one region, the function is modified somewhat. Because there may be a tendency for the algorithm to converge to a configuration of sources which minimize the field to a much greater extent at one location vs. another, a penalty term $(\mathbf{K})$ is added. 
In Equation 6.4 the difference in the $\vec{B}$ components are penalized rather than simply considering the differential as

$$
B_{A 1-A 2}=B_{A 1}-B_{A 2}
$$

For the GeoSurv II application, the magnitude differential is sufficient because the system magnetometers only measure the scalar magnitude. In order to keep the algorithm general to systems that may be using vector sensors, it is necessary to ensure that the individual components of the average field are equalized as well to ensure that

$$
\vec{B}_{A 1}-\vec{B}_{A 2}=0
$$

Accordingly, the vector differential was used.

\subsubsection{Constraints}

Only boundary constraints were considered for the OFA cases discussed in this thesis. The fmincon function, however, is capable of supporting equality, and inequality constraint functions. These constraints were not considered here because of the complexity of applying inequality constraints to several independent sources of interference. If several sources of interference were allowed to exist in the same constraint area, the OFA solver may determine that the optimal configuration places the two sources at the same point, but with opposite orientation which effectively cancels the two out. To circumvent this using boundary constraints requires that a system be sectioned off into $\mathrm{N}$-independent and non-overlapping constraint regions; this is not ideal, and may limit the attenuation that is achievable by the OFA solver. 


\subsection{OFA Verification Experiment}

\subsubsection{Test Configuration}

Three MoDEM modelled dipole sources, (2DP, 3DPa and 3DPb, discussed in the previous chapter) were used for experimental validation of the OFA algorithm. The sources were arranged in constraint regions as described by Figure 6.2. Ten cases were considered; five for a $1 \mathrm{~cm} \mathrm{x} 1 \mathrm{~cm}$ area of optimization and five for a $5 \mathrm{~cm} \times 5 \mathrm{~cm}$ area of optimization under the following initial conditions and test constraints:

- A: Full in-plane translation and rotation. All sources were initially positioned at the centre of their respective constraint regions with orientations of $90^{\circ}, 0^{\circ}$, and $90^{\circ}$ respectively for the $2 \mathrm{DP}, 3 \mathrm{DPa}$ and $3 \mathrm{DPb}$ field sources.

- B: Full in-plane translation and rotation. All sources were initially positioned at the centre of their respective constraint regions with orientations of $90^{\circ}$ for the three field sources.

- C: Full in-plane translation and rotation. All sources were initially positioned at the centre of their respective constraint regions with orientations of $0^{o}$ for the three field sources.

- D: Fixed X-axis translation; full $\mathrm{Y}$-axis translation and in-plane rotation. All sources were initially positioned at the centre of their respective constraint regions with orientations of $0^{\circ}$ for the three field sources.

- E: Fixed in-plane translation; full in-plane rotation. All sources were initially positioned at the centre of their respective constraint regions with orientations of $0^{\circ}$ for the three field sources. 


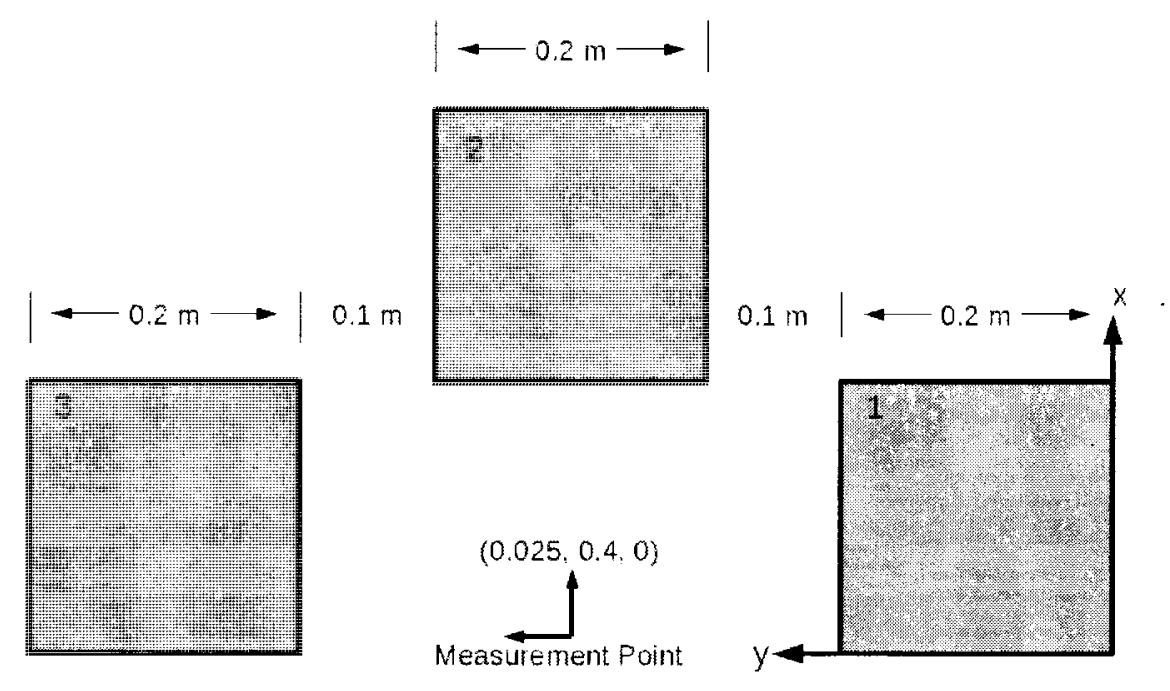

Figure 6.2: Verification Test Configuration. Each of the three constraint regions measures $0.2 \mathrm{~m}$ square; regions 1 through 3 contain the $2 \mathrm{DP}, 3 \mathrm{DPa}$, and $3 \mathrm{DPb}$ specimens respectively. The optimization area is a square region centered on the measurement point.

\subsubsection{Results}

Tables 6.1 and 6.2 and Figures 6.3 through 6.8 compare the theoretical and experimental results for the ten test cases. The values in Table 6.2 were calculated by taking the difference in the measured and estimated initial and optimized values.

\section{Error}

In Section 5.5 the modelling error for the 2DP, 3DPa, and 3DPb field sources was discussed. Analyzing the modelling errors for independent sources is less complicated than analyzing the combined errors of the three sources considered for the OFA verification tests. The error bounds shown in Figures 6.3 through 6.8 estimate the sum of the average modelling error considered for each independent source. The estimate assumes that the modelling error determined in Section 5.5 decays at a rate of $\mathrm{R}^{3}$, as the separation between source and observation point increases. On this basis, the modelling error for each source was extrapolated for the respective positions of 
the sources under the ten test conditions. By adding these estimates, a measure of the uncertainty in the model was determined.

\subsubsection{Analysis}

For all ten cases, with the exception of case $E(5 \mathrm{~cm})$, the estimated field attenuation matched very closely with the measured values. Although the prediction of the pre and post field intensities were in greater error than was expected, the predictions were consistently greater than the measurements. The estimates, therefore, may be considered to be conservative. On average the error was approximately $580 \mathrm{nT}$ for the initial estimate and $355 \mathrm{nT}$ for the optimized estimates. The attenuation, which consideres the difference between pre and post field intensities yielded an average error of $450 \mathrm{nT}$.

The configuration that was used to verify the algorithm did not result in an average field intensity that meets GeoSurv II requirements. This was not unexpected as the magnetic sources that were used are far stronger and were configured much closer to the region of optimization than those of GeoSurv II. The results do show quite clearly that the OFA algorithm can be used to predict and minimize the magnetic interference produced by a set of magnetic sources that were modelled using dipole equivalence techniques. The following section provides further analysis of the OFA algorithm using simulated test cases. 
Table 6.1: OFA Verification Testing (all values in $\mathrm{nT}$ )

\begin{tabular}{|c|c|c|c|c|}
\hline $\begin{array}{c}\text { Test } \\
\text { Case }\end{array}$ & $\begin{array}{c}\text { Initial } \\
\text { Estimate }\end{array}$ & $\begin{array}{c}\text { Initial } \\
\text { Measurement }\end{array}$ & $\begin{array}{c}\text { Optimized } \\
\text { Estimate }\end{array}$ & $\begin{array}{c}\text { Optimized } \\
\text { Measurement }\end{array}$ \\
\hline \hline $\mathrm{A}(1 \mathrm{~cm})$ & 30906 & 31600 & 346 & 680 \\
\hline $\mathrm{B}(1 \mathrm{~cm})$ & 21372 & 21528 & 393 & 934 \\
\hline $\mathrm{C}(1 \mathrm{~cm})$ & 10700 & 12040 & 40 & 516 \\
\hline $\mathrm{D}(1 \mathrm{~cm})$ & 10700 & 12040 & 168 & 488 \\
\hline $\mathrm{E}(1 \mathrm{~cm})$ & 10700 & 12040 & 791 & 1437 \\
\hline $\mathrm{A}(5 \mathrm{~cm})$ & 31192 & 31600 & 224 & 680 \\
\hline $\mathrm{B}(5 \mathrm{~cm})$ & 21700 & 21528 & 549 & 934 \\
\hline $\mathrm{C}(5 \mathrm{~cm})$ & 10987 & 12040 & 169 & 516 \\
\hline $\mathrm{D}(5 \mathrm{~cm})$ & 10987 & 12040 & 847 & 488 \\
\hline $\mathrm{E}(5 \mathrm{~cm})$ & 10987 & 12040 & 4308 & 1437 \\
\hline
\end{tabular}

Table 6.2: Theoretical vs. Experimental Attenuation (all values in nT)

\begin{tabular}{|c|c|c|c|}
\hline $\begin{array}{c}\text { Test } \\
\text { Case }\end{array}$ & $\begin{array}{c}\text { Attenuation } \\
\text { Estimate }\end{array}$ & $\begin{array}{c}\text { Attenuation } \\
\text { Measurement }\end{array}$ & $\begin{array}{c}\text { Percent } \\
\text { Error }\end{array}$ \\
\hline \hline $\mathrm{A}(1 \mathrm{~cm})$ & 30560 & 30920 & -1.18 \\
\hline $\mathrm{B}(1 \mathrm{~cm})$ & 20979 & 20594 & 1.84 \\
\hline $\mathrm{C}(1 \mathrm{~cm})$ & 10660 & 11524 & -8.11 \\
\hline $\mathrm{D}(1 \mathrm{~cm})$ & 10532 & 11552 & -9.68 \\
\hline $\mathrm{E}(1 \mathrm{~cm})$ & 9909 & 10603 & -7 \\
\hline $\mathrm{A}(5 \mathrm{~cm})$ & 30968 & 30920 & 0.15 \\
\hline $\mathrm{B}(5 \mathrm{~cm})$ & 21151 & 20594 & 2.63 \\
\hline $\mathrm{C}(5 \mathrm{~cm})$ & 10818 & 11524 & -6.53 \\
\hline $\mathrm{D}(5 \mathrm{~cm})$ & 10140 & 11552 & -13.93 \\
\hline $\mathrm{E}(5 \mathrm{~cm})$ & 6679 & 10603 & -58.75 \\
\hline
\end{tabular}




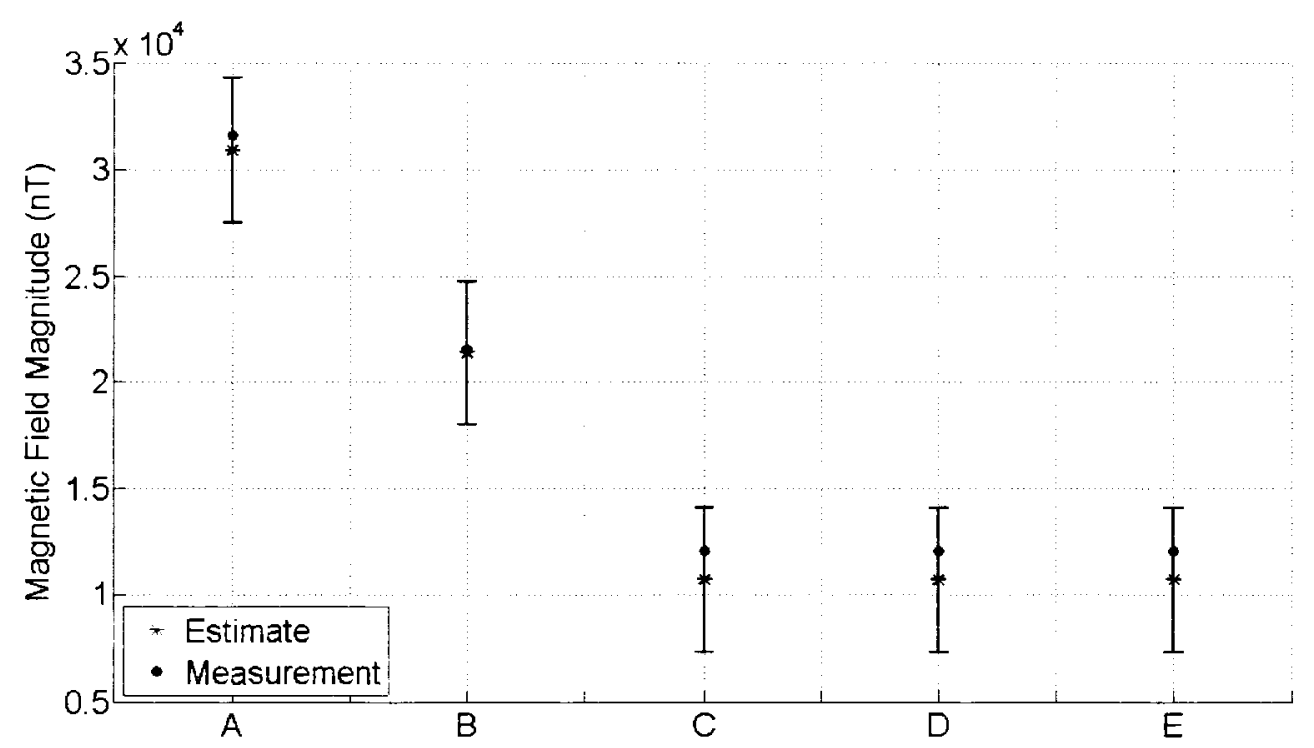

Figure 6.3: Initial Field Measure vs. Model $-1 \mathrm{~cm} \times 1 \mathrm{~cm}$ Area. For all cases the measured value of the field fell within the estimate error bounds.

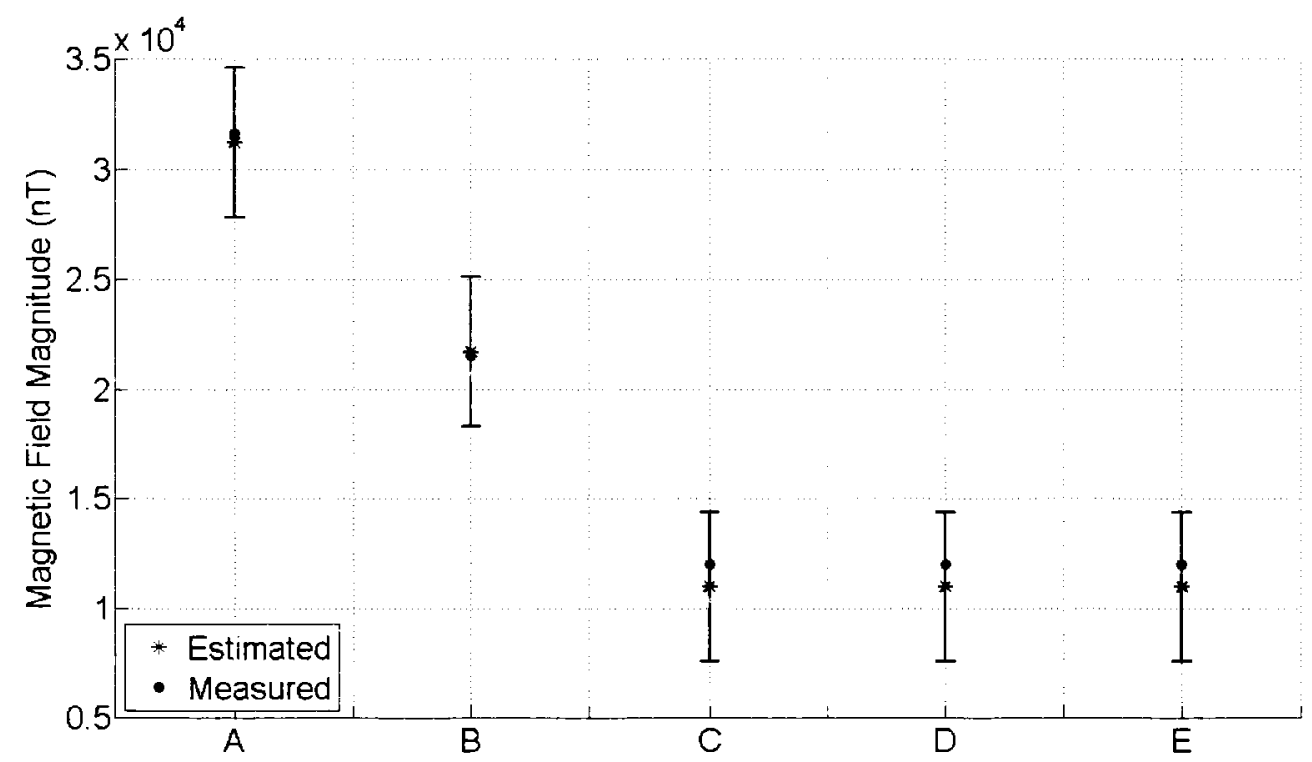

Figure 6.4: Initial Field Measure vs. Model $-5 \mathrm{~cm} \mathrm{x} 5 \mathrm{~cm}$ Area. For all cases the measured value of the field fell within the estimate error bounds. 


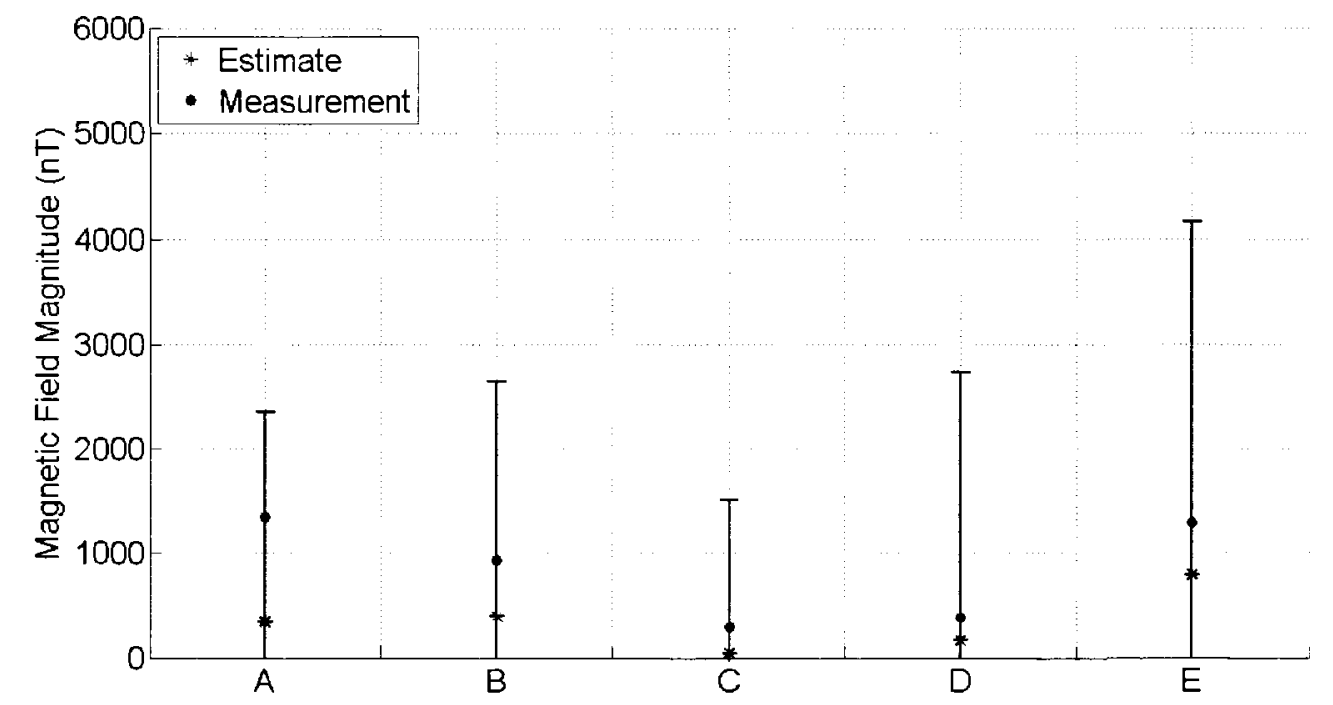

Figure 6.5: Optimized Field Measure vs. Model $-1 \mathrm{~cm}$ x $1 \mathrm{~cm}$ Area. For all cases the measured value of the field fell within the estimate error bounds.

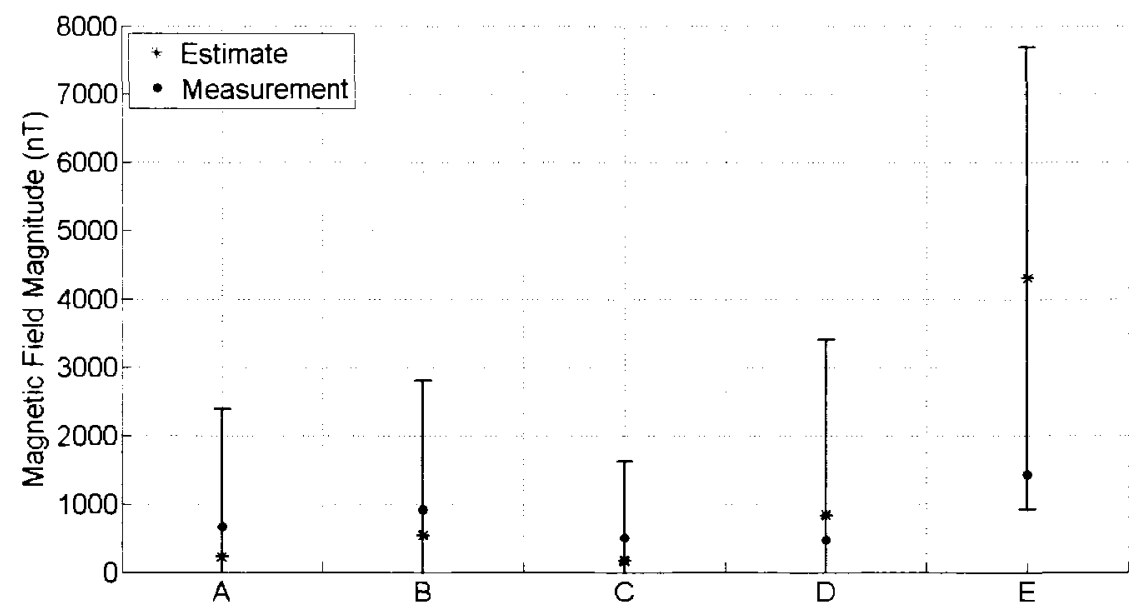

Figure 6.6: Optimized Field Measure vs. Model - $5 \mathrm{~cm}$ x $5 \mathrm{~cm}$ Area. For all cases the measured value of the field fell within the estimate error bounds. The poor result observed in case $\mathrm{E}$ is attributed to an inaccuracy in positioning and orienting the sources in the optimal configuration. 


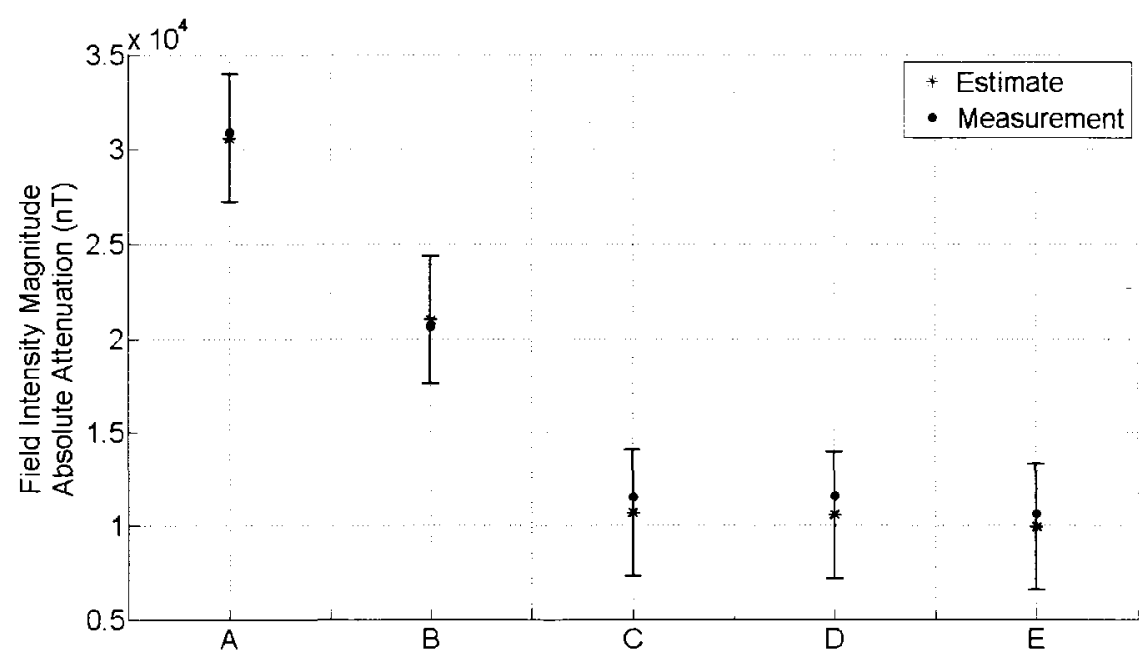

Figure 6.7: Field Attenuation Measure vs. Model - $1 \mathrm{~cm} \times 1 \mathrm{~cm}$ Area. Excellent agreement was observed between the modelled and measured field values for the five test cases. For all cases the measured value of the field fell within the estimate error bounds.

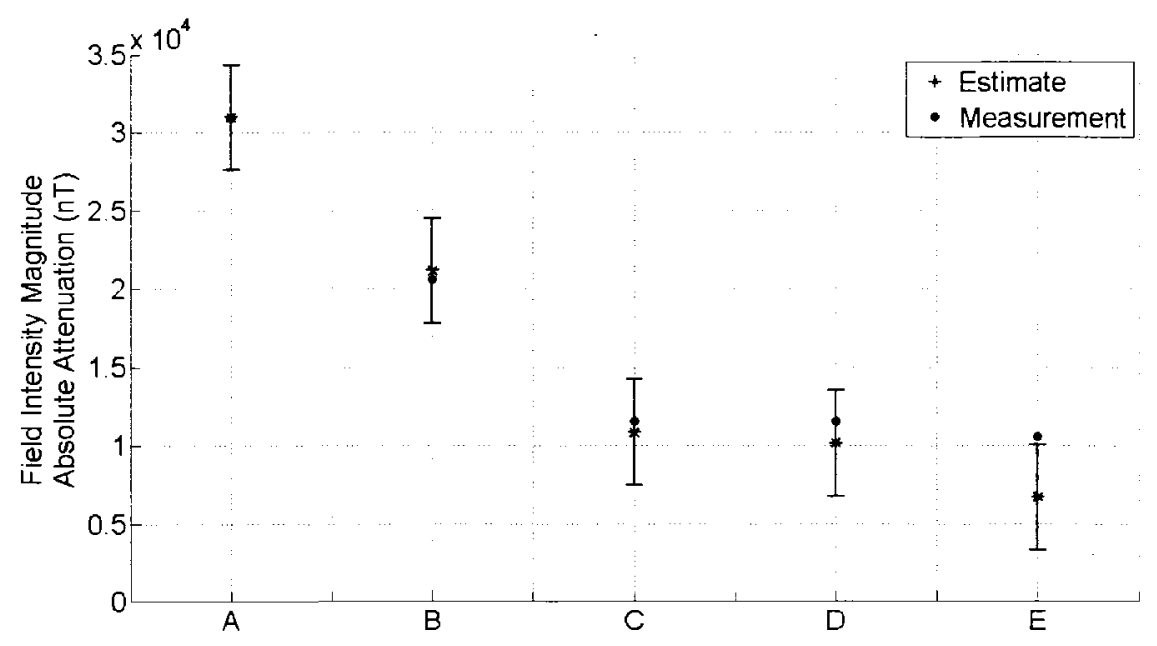

Figure 6.8: Field Attenuation Measure vs. Model - 5cm x 5cm Area. For all cases the measured value of the field fell within the estimate error bounds. The poor result observed in case $\mathrm{E}$ is attributed to an inaccuracy in positioning and orienting the sources in the optimal configuration. 


\subsection{OFA Analysis}

\subsubsection{Attenuation vs. Spatial Field Stability}

Figure 6.9 describes the configuration of sources considered for simulated investigations of the OFA algorithm in terms of comparing spatial field stability and attenuation vs. area of interest. Two cases were considered using this configuration. The first considers that all sources may be freely positioned at any X,Y coordinate and in-plane rotation within the respective constraint region. The second considers that sources one through four are fixed and only the remaining sources may be freely configured. OFA optimization areas of $0.001 \mathrm{~m} \times 0.001 \mathrm{~m}, 0.02 \mathrm{~m} \mathrm{x} 0.02 \mathrm{~m}, 0.04 \mathrm{~m} \mathrm{x}$ $0.04 \mathrm{~m}, 0.06 \mathrm{~m} \times 0.06 \mathrm{~m}$, and $0.08 \mathrm{~m} \times 0.08 \mathrm{~m}$ were considered.

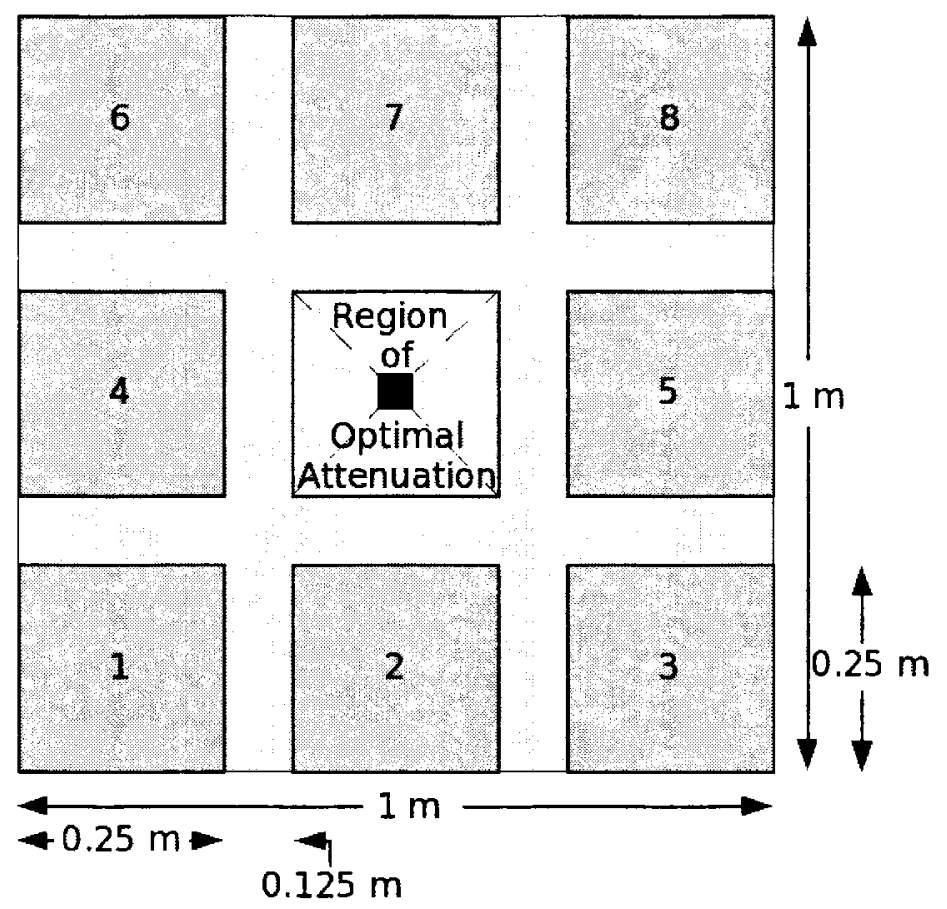

Figure 6.9: Attenuation vs. Spatial Field Stability Teats Configuration. Eight sources were considered for the study of attenuation factor vs. optimization area. Each source is considered identical and positioned in the center of its constraint region. 


\section{Trade-off Between Spatial Stability and Field Attenuation}

The percent attenuation is defined as,

$$
\% \text { Attenuation }=100 \times \frac{\text { Optimal } B_{\text {mean }}}{\text { Initial } B_{\text {mean }}}
$$

where Optimal and Initial $B_{\text {mean }}$ refer to average field calculated over the optimization area.

The spatial field stability is compared on the basis of a metric defined as,

$$
\text { Stability Metric }=\frac{\text { Optimal } B_{\text {centre }}}{\text { Optimal } B_{\text {mean }}}
$$

where Optimal $B_{\text {centre }}$ refers to the value of the magnetic field intensity at the centre of the optimization area, Optimal $B_{\text {mean }}$ is the average field intensity over the area surrounding it. A small stability metric indicates that there is a large variation in the field intensity over the optimization area. Ideally we would like to see the stability metric approach a value of 1 which indicates a spatial invariance of the field.

Figures 6.10 and 6.11 describe the relationship between the field attenuation and the area of optimization. Figure 6.10 plots the optimized average field intensity vs. the optimization area for the Full Freedom OFA trial case. Figure 6.11 plots the percent attenuation, Equation 6.7, vs. the optimization area for both the Full Freedom and Constrained OFA Trials. In both figures the degree of attenuation tends to decrease with respect to the area of optimized field. However this trend is far less significant for the Full Freedom OFA trial shown in Figure 6.11 which indicates that, although the average attenuation may increase with the size of the optimization area, if sufficient degrees of freedom (source configuration flexibility) are available then the ratio of initial field to optimized field remains relatively constant. 


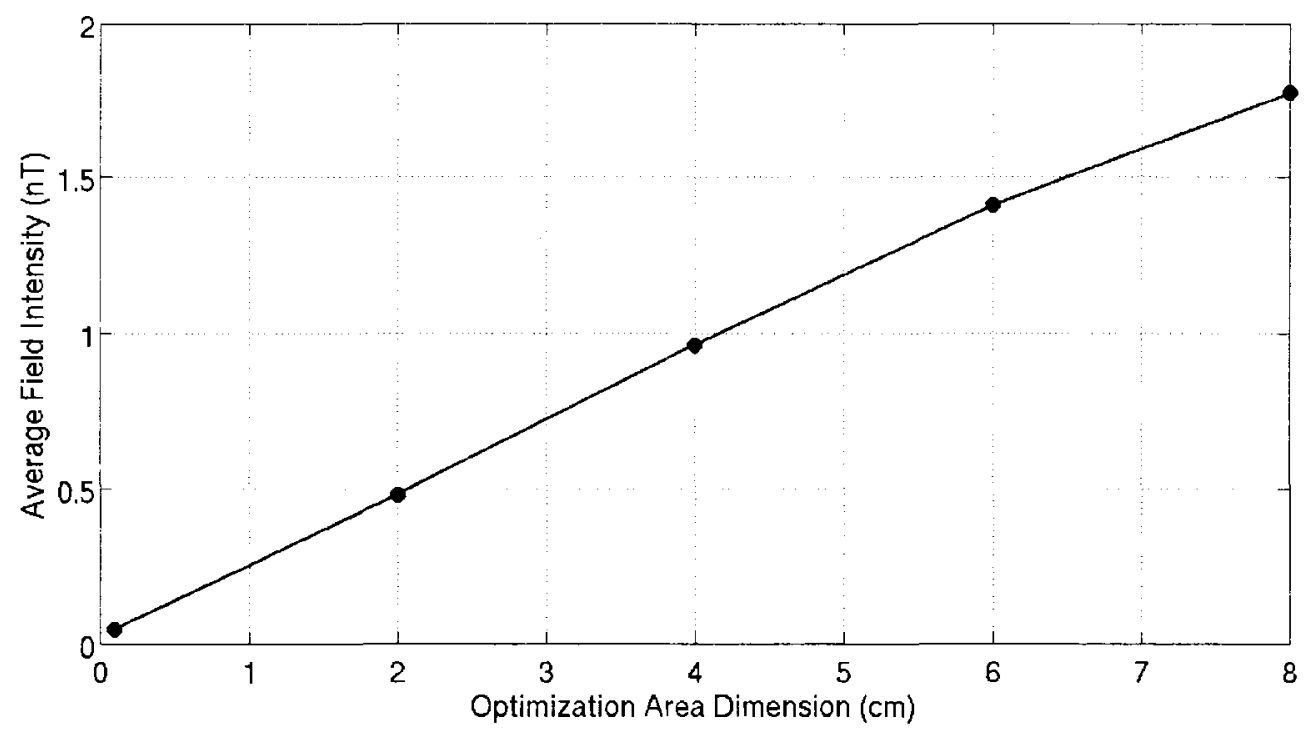

Figure 6.10: Average (attenuated) Field Intensity vs. Area of Optimization (Full Freedom Case). $\|\vec{B}\|$ increases at a rate of $0.215 \mathrm{nT} /$ Optimization Area Dimension.

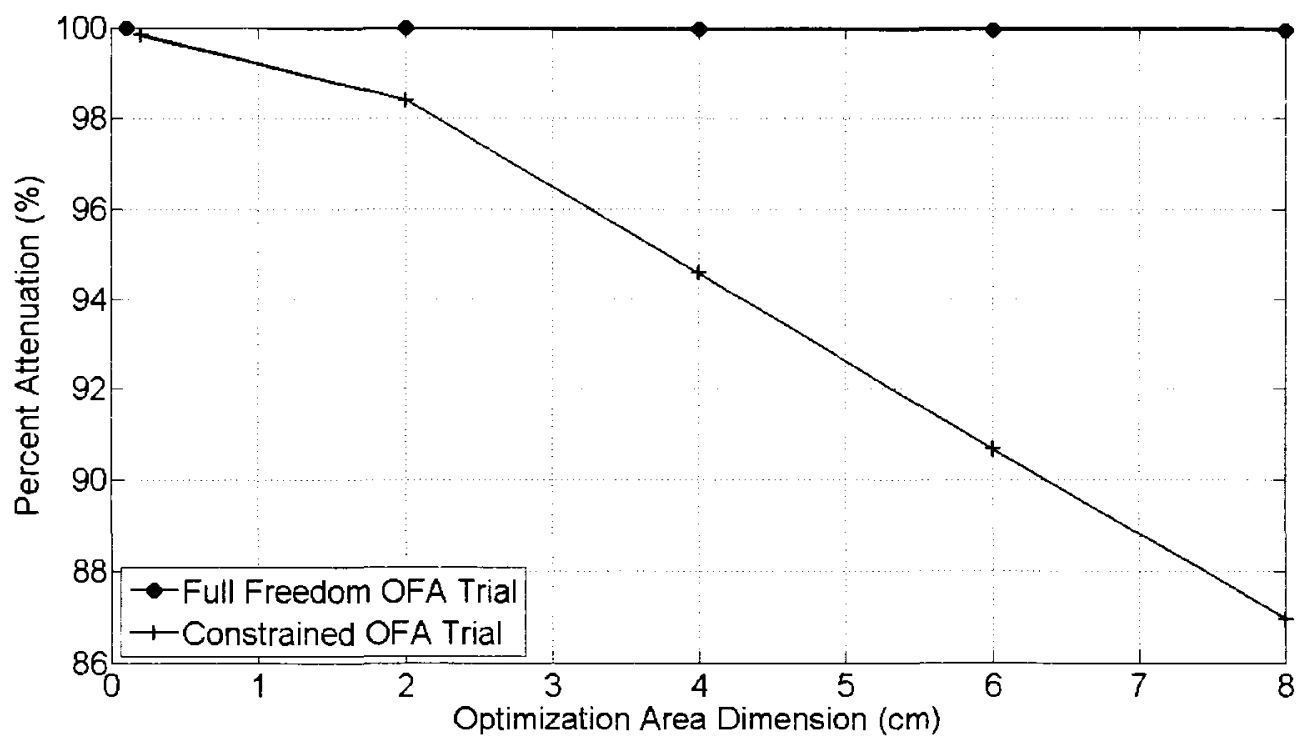

Figure 6.11: Percent Attenuation vs. Area of Optimization. The percent attenuation was effectively constant for the full freedom OFA trial; the constrained case was discovered to decrease at a rate of approximately. 1.67\%/Optimization Area Dimension. 
Figure 6.12 compares the stability factor vs. the size of the optimization area. The stability factor, in this case, considers that Optimal $B_{\text {mean }}$ is calculated over the entire $0.25 \mathrm{~m} \times 0.25 \mathrm{~m}$ region illustrated in Figure 6.9 . It is shown that the OFA algorithm objective of spatial field stability is improved by increasing the size of the optinization area. Furthermore, greater degrees of freedom result in increased field stability.

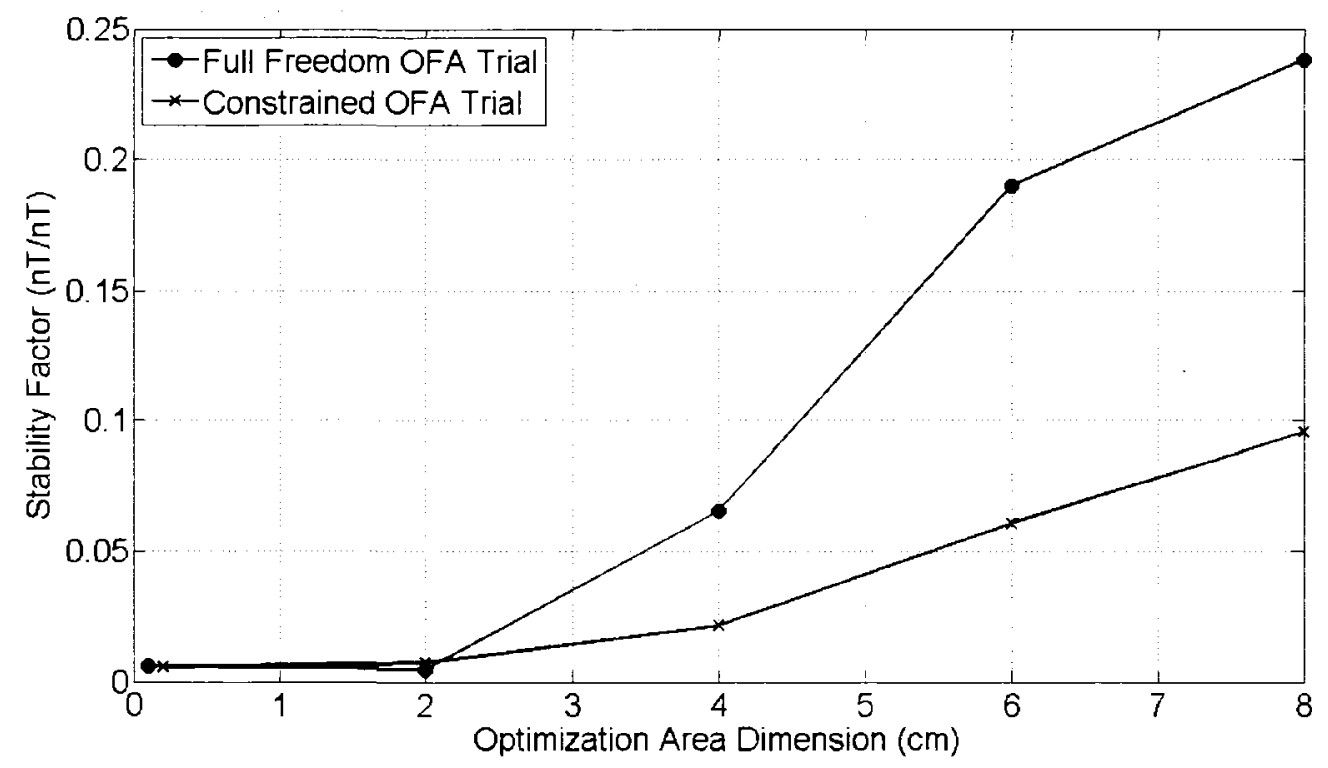

Figure 6.12: Stability Metric vs. Area of Optimization. The stability metric was found to increase at a faster rate for the full freedom OFA trial than the constrained trial. Both, however, indicate that increased areas of optimization improve the spatial field stability.

The spatial field stability improvement resulting from larger areas of optimization are illustrated in Figures 6.13 through 6.15. These figures show the field intensity contour and the field intensity envelope over the $0.25 \mathrm{~m} \times 0.25 \mathrm{~m}$ region of optimal attenuation. The field intensity envelope is a set of slices through the region which illustrate the improvement in spatial field stability vs. the size of the optimization area. It is clear that as the optimization area increased the spatial field stability increased as well. Between the $1 \mathrm{~mm} \times 1 \mathrm{~mm}$ and $5 \mathrm{~cm} \times 5 \mathrm{~cm}$ cases the maximum field 
value decreased by a factor of ten, and the overall distribution approached a desirable shallow bowl shape.
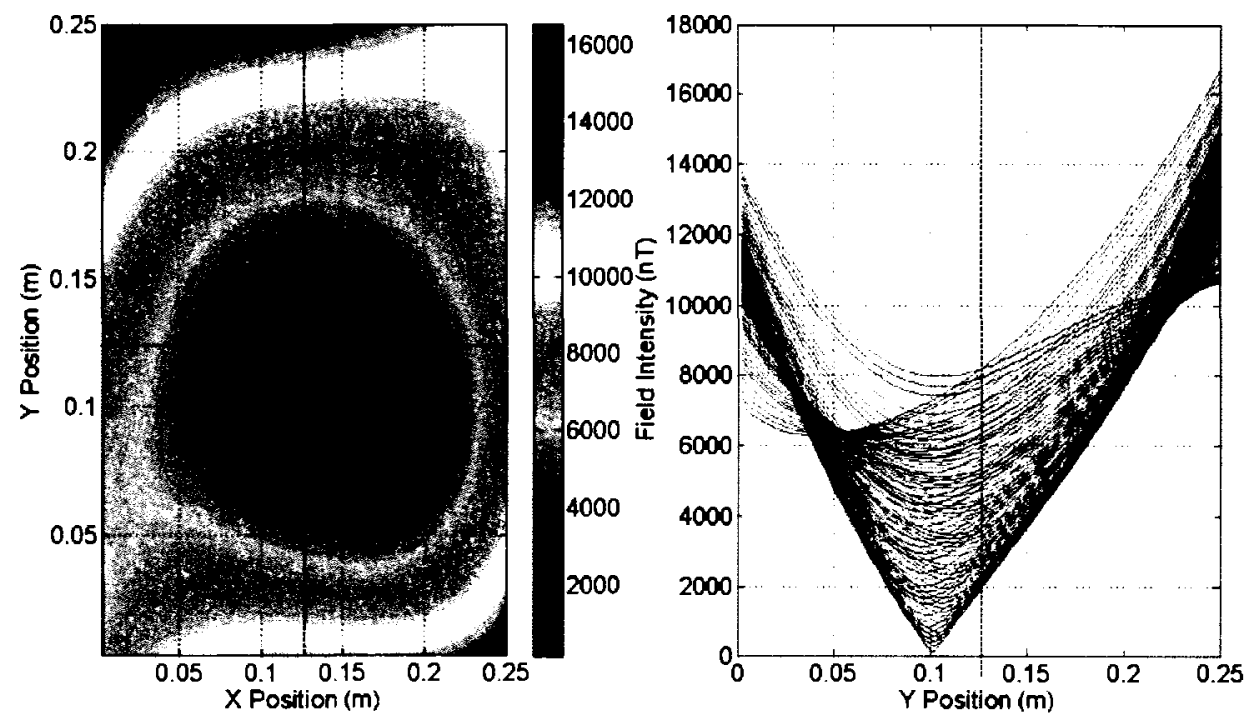

Figure 6.13: Initial Field Distribution - Full Flexibility Case. Initially, the field was not minimized at the desired point. Furthermore, a large spatial field intensity gradient is present; this indicates poor spatial field stability. 

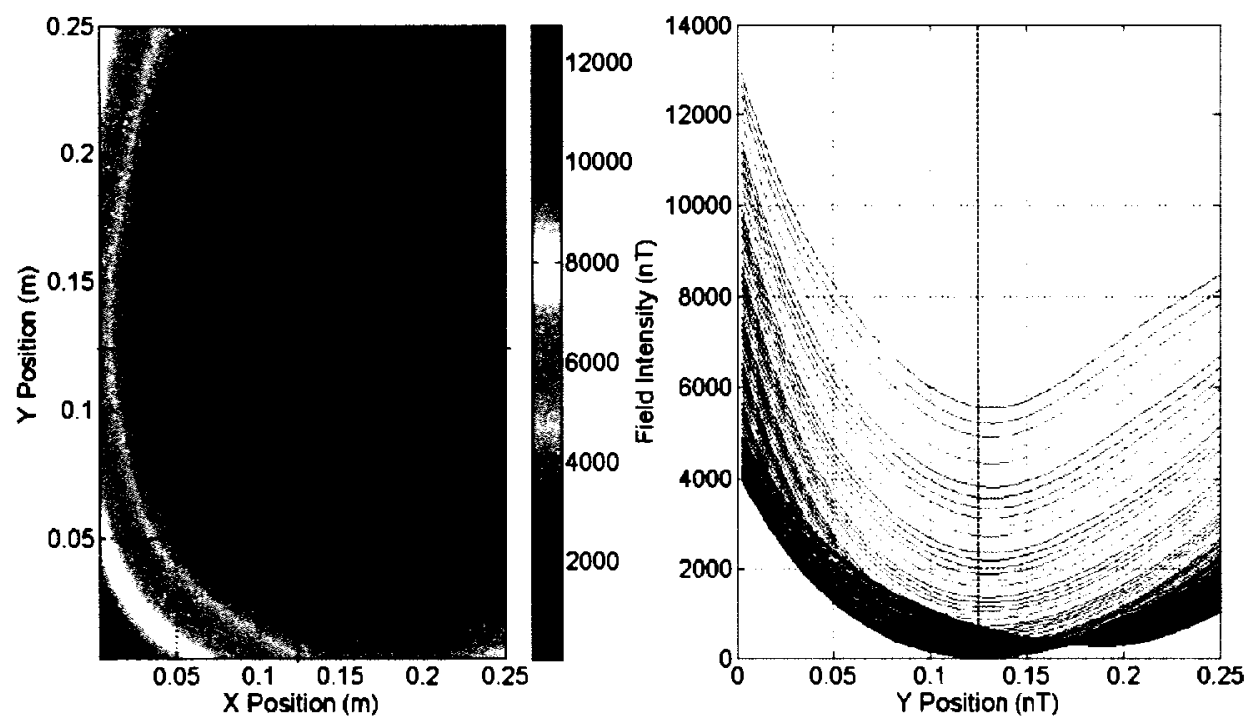

Figure 6.14: $1 \mathrm{~mm} \times 1 \mathrm{~mm}$ Optimization Area Field Distribution - Full Flexibility Case. A $1 \mathrm{~mm} \times 1 \mathrm{~mm}$ area of optimization improved the spatial field stability in the optimization area. Over the full $0.25 \mathrm{~m} \times 0.25 \mathrm{~m}$ region, however, a large field intensity gradient is still present.
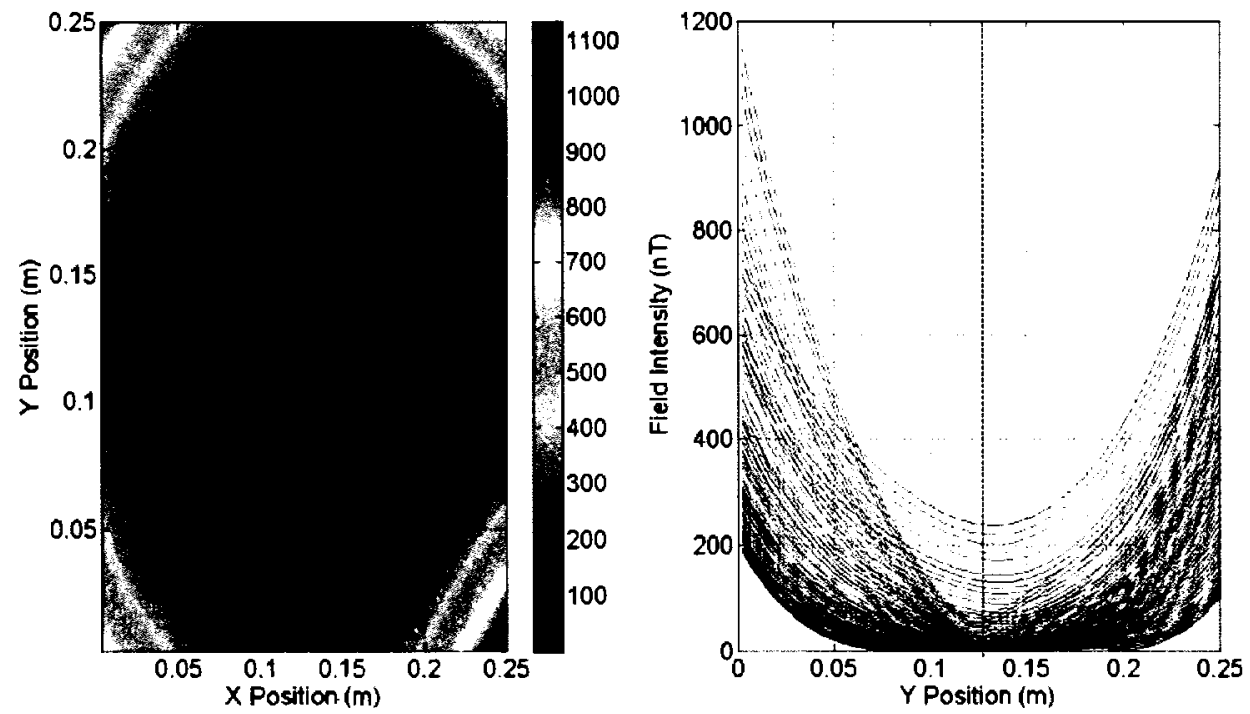

Figure 6.15: $5 \mathrm{~cm} \times 5 \mathrm{~cm}$ Optimization Area Field Distribution - Full Flexibility Case. By considering a $5 \mathrm{~cm}$ x $5 \mathrm{~cm}$ optimization area, the field intensity is minimized at the desired location and the spatial field stability is significantly improved vs. the previous two figures. 


\subsubsection{GeoSurv II Multi-Objective Field Equalization}

Figure 6.16 and Table 6.3 outlines the constraint regions considered for an OFA analysis of the primary flight control actuators on GeoSurv II. For each of the five control surfaces the Hitec HSR-5995TG servo MoDEM model was used. All sources were considered to be oriented in the XY plane and were given full rotational freedom.

Table 6.3: UAV Planar Boundary Constraints for Figure 6.16

\begin{tabular}{|c|c|c|c|c|c|c|}
\hline $\begin{array}{c}\text { Constraint } \\
\text { Region }\end{array}$ & $\begin{array}{c}\mathrm{X}_{\text {low. }} \\
(\mathrm{m})\end{array}$ & $\begin{array}{c}\mathrm{X}_{\text {upp. }} \\
(\mathrm{m})\end{array}$ & $\begin{array}{c}\mathrm{Y}_{\text {low. }} \\
(\mathrm{m})\end{array}$ & $\begin{array}{c}\mathrm{Y}_{\text {upp. }} \\
(\mathrm{m})\end{array}$ & $\begin{array}{c}\mathrm{Z}_{\text {low. }} \\
(\mathrm{m})\end{array}$ & $\begin{array}{c}\mathrm{Z}_{\text {upp. }} \\
(\mathrm{m})\end{array}$ \\
\hline \hline 1. & 0.330 & 1.727 & 0.051 & 0.102 & 0 & 0 \\
\hline 2. & 3.140 & 4.547 & 0.051 & 0.102 & 0 & 0 \\
\hline 3. & 1.727 & 1.727 & 2.540 & 2.540 & 0.152 & 0.559 \\
\hline 4. & 3.140 & 3.140 & 2.540 & 2.540 & 0.152 & 0.559 \\
\hline 5. & 2.057 & 2.819 & 2.794 & 2.845 & 0 & 0 \\
\hline
\end{tabular}




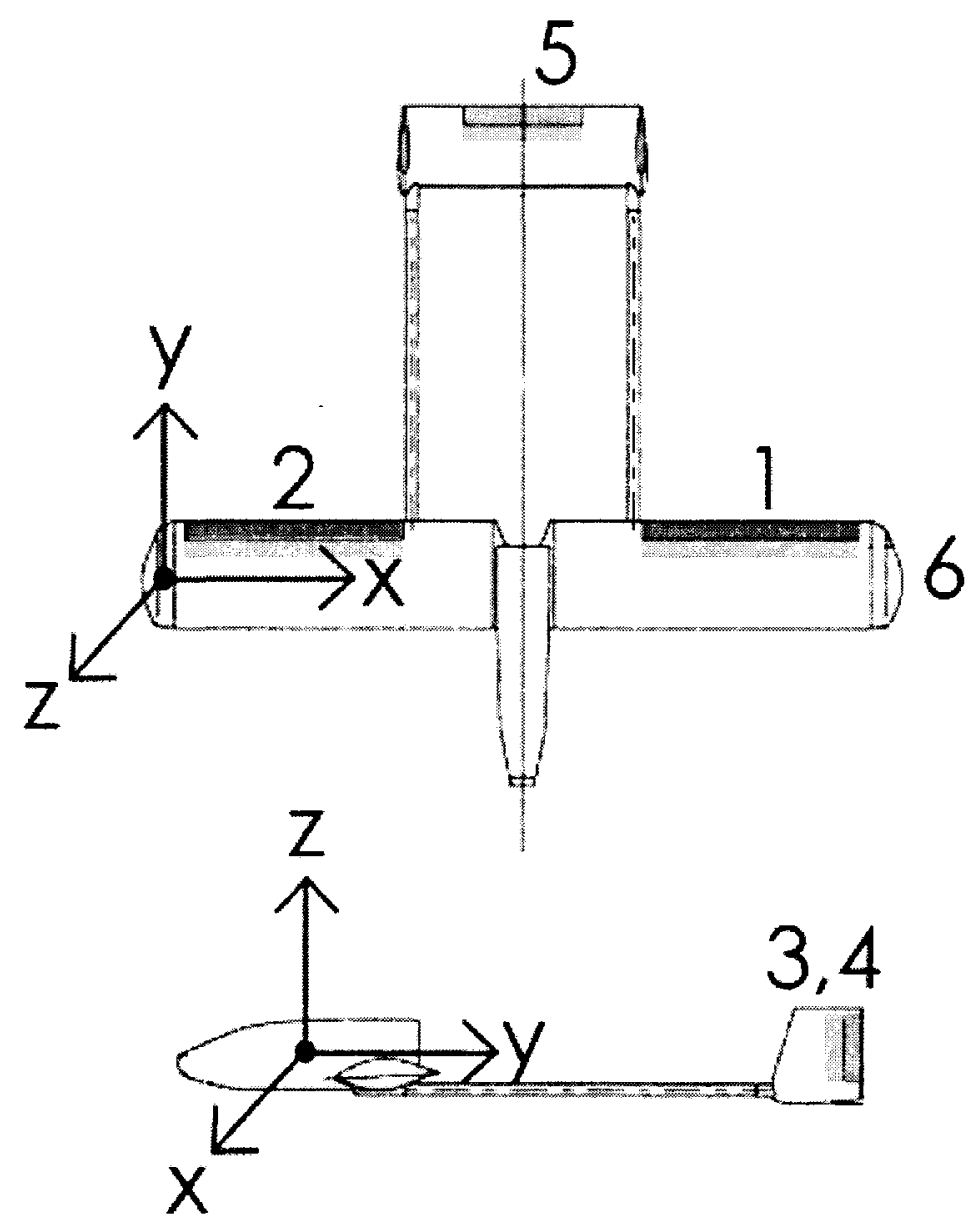

Figure 6.16: Constraint Regions for the GeoSurv II Primary Flight Control Actuators. The upper and lower bounds for each of the regions is described in Table 6.3; the right wing magnetometer was located at a distance of $4.9 \mathrm{~m}$ along the $\mathrm{x}$-axis. All constraint regions (grey areas) represent feasible positions for the servo actuators. 
The objective of the test was to compare left to right field equalization between the single and multi-source optimization methods. The following test cases were considered:

- Case 1: Near symmetrical initial actuator configuration multi objective optimization. The five sources were placed near the centre of their respective constraint region and all were oriented at zero degrees.

- Case 2: Asymmetrical initial actuator configuration multi objective optimization. The five sources were placed near the centre of their respective constraint region. the left aileron and rudder actuators were mounted at zero degrees, as was the elevator; the remaining actuators were oriented at ninety degrees.

- Case 3: Asymmetric initial actuator configuration single objective optimization. The asymmetric configuration was used to optimize the field at the left wingtip magnetometer only.

For each of the three cases listed above, OFA analysis for optimization areas of $0.01 \mathrm{~m}$ $\mathrm{x} 0.01 \mathrm{~m}, 0.03 \mathrm{~m} \times 0.03 \mathrm{~m}, 0.05 \mathrm{~m} \times 0.05 \mathrm{~m}, 0.07 \mathrm{~m} \times 0.07 \mathrm{~m}$, and $0.09 \mathrm{~m} \times 0.09 \mathrm{~m}$ were considered for single and multi-area objective functions as described in Equations 6.1 and 6.2 respectively.

\section{Results: Magnetic Field Equalization}

The results of field equalization investigation for GeoSurv II are listed in Table 6.4. In Figure 6.17 the average field differential described by Equation 6.4 is plotted for Cases 1 and 2 vs. the area of optimization. Sinilarly to the relationship between the achievable attenuation and area of optimization, discussed in the previous section, the achievable field differential tends to increase with respect to the size of the optimization area. However for the the GeoSurv II investigation discussed here, all 
five optimization areas meet the $0.1 \mathrm{nT}$ interference requirement. Furthermore, with the exception of the largest $(0.09 \mathrm{~m} \times 0.09 \mathrm{~m})$ area, the differential met the $0.01 \mathrm{nT}$ requirement for time variant sources of magnetic interference as well.

Table 6.4: Left - Right Estimated Field Magnitude Differential (all values in $11 \mathrm{~T}$ )

\begin{tabular}{|c|c|c|c|c|c|c|}
\hline $\begin{array}{c}\text { Constraint } \\
\text { Area }\left(\mathrm{m}^{2}\right)\end{array}$ & $\begin{array}{c}\text { Case 1. } \\
\text { Initial. }\end{array}$ & $\begin{array}{c}\text { Case 1. } \\
\text { Opt. }\end{array}$ & $\begin{array}{c}\text { Case 2. } \\
\text { Ini. }\end{array}$ & $\begin{array}{c}\text { Case 2. } \\
\text { Opt. }\end{array}$ & $\begin{array}{c}\text { Case 3. } \\
\text { Ini }\end{array}$ & $\begin{array}{c}\text { Case 3 } \\
\text { Opt. }\end{array}$ \\
\hline \hline $0.01 \times 0.01$ & 3.700 & -0.001 & 8.840 & -0.001 & 8.840 & 0.240 \\
\hline $0.03 \times 0.03$ & 2.960 & -0.003 & 8.340 & -0.001 & 8.300 & 0.213 \\
\hline $0.05 \times 0.05$ & 2.200 & -0.007 & 7.760 & -0.007 & 7.730 & 1.302 \\
\hline $0.07 \times 0.07$ & 1.460 & -0.009 & 7.600 & -0.007 & 7.220 & 1.263 \\
\hline $0.09 \times 0.09$ & 0.710 & -0.012 & 6.700 & -0.012 & 6.700 & 1.233 \\
\hline
\end{tabular}

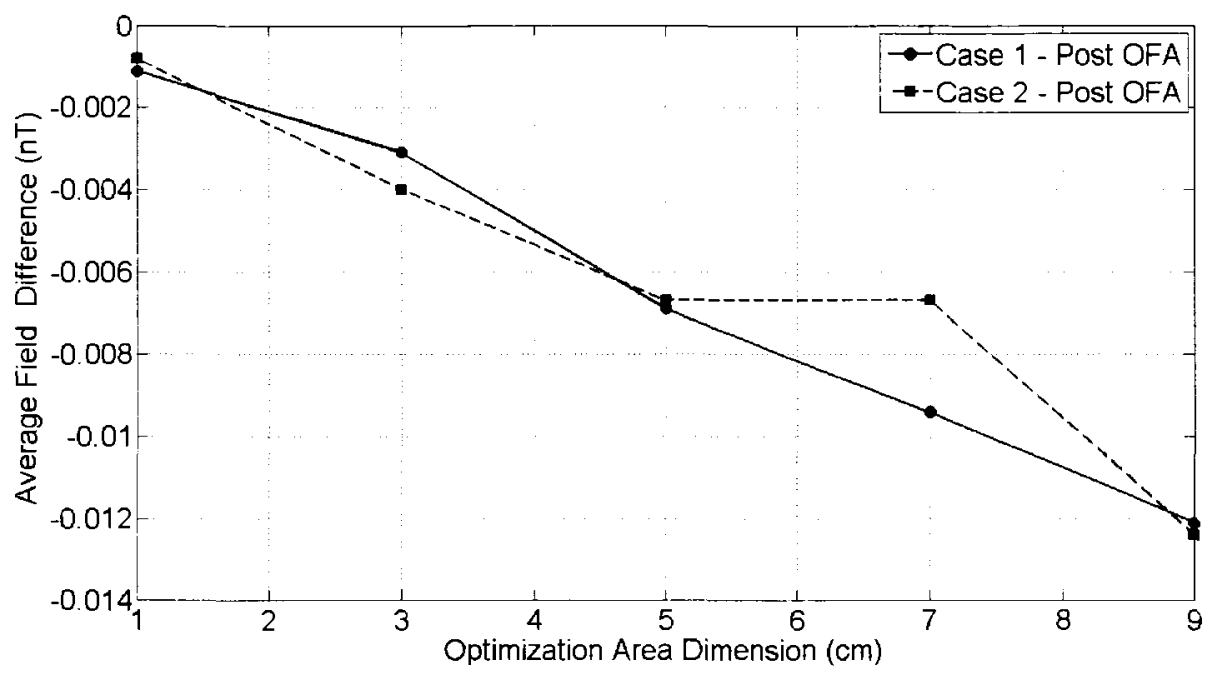

Figure 6.17: Post-Optimized Left-Right Field Differential. For Cases 1 and 2, the average differential increases at a rate of approximately 0.0014 nT/Optimization Area Dimension.

Trial Case 3, which considered the single area OFA objective, did not result in suitable field equalization between the two magnetometers. Although the results 
listed in Table 6.4 indicate that Trial Case 3 did reduce the field differential this can be attributed to the fact that optimizing for the area surrounding the left wingtip magnetometer would result in a configuration of sources that exhibit some left/right symmetry. The results of this investigation clearly show that by considering multiple area optimization objective functions is far more effective for ensuring minimum magnetic field differentials.

\subsection{OFA for Use on GeoSurv II}

Experimental validation of OFA for GeoSurv II was not completed because only two Hitec servos were available for testing, actual constraint regions have not yet been established by the design team and the magnetic measurement apparatus used in this thesis is not sensitive enough for a conclusive test on the suitability of the algorithm. The validation testing discussed in Section 6.4 and the theoretical investigations discussed in Section 6.5 indicate that the OFA algorithm meets the stated objectives of attenuating, spatially stabilizing, and equalizing the magnetic interference generated by a system at several areas of interest. Considering the GeoSurv II actuating system, it is concluded that Optimal Field Attenuation should be used to meet the interference tolerance of $0.1 \mathrm{nT}$ for time invariant fields. Future work is necessary to extend the algorithm to time variant magnetic interference. 


\section{Chapter 7}

\section{Conclusions and Recommendations}

\subsection{Summary}

In this thesis the problem of attenuating magnetic interference in an autonomous geomagnetic surveying UAV (GeoSurv II) was presented. Geomagnetic surveying involves measuring the environmental magnetic field at very high resolution $(0.01 \mathrm{nT})$; the achievable resolution is quickly eroded by the addition of magnetic interference, produced by the aircraft, to the measured total field. The actuation system on the aircraft is believed to be the greatest contributor to magnetic interference because of the combination of electrical power systems, moving parts, and common actuator components (DC inotors, ferrite gears, etc...) inherent in the system.

A survey of possible low magnetic actuator alternatives to DC servos was completed, however, it was decided that the best course of action for the overall development of the GeoSurv II was to proceed with electromagnetic actuators. Existing magnetic shielding solutions were deemed to be unsuitable for use on the aircraft; passive magnetic shields will alter the local field gradient and the complexity of active shielding control systems are beyond the scope of this project. A novel strategy called optimal field attenuation (OFA) was selected for use on the aircraft. OFA involves 
modelling sources of interference using dipole equivalence methods and establishing an optimal configuration (position and orientation) of actuation system components such that their combined interference is minimized at the magnetometer location. Using a custom magnetic measurement apparatus, made in-house, the modelling and OFA algorithms were experimentally validated. Based on the work discussed in this thesis, it can be concluded that OFA is a viable method for minimizing magnetic interference produced by a collection of field sources in systems using magnetometers.

This chapter will present the conclusions reached, and recommendations for fut ure work regarding the research objectives defined for this thesis:

1. Research magnetic interference attenuation strategies currently in use; investigate the suitability of the strategies for use on GeoSurv II.

2. Investigate low magnetic actuation options for use on GeoSurv II.

3. Develop and experimentally verify a modelling strategy for estimating the intensity of magnetic interference generated by GeoSurv II.

4. Develop and experimentally verify a magnetic interference attenuation strategy.

\subsection{Research Conclusions}

\subsubsection{Attenuation Strategies for GeoSurv II}

1. Traditional magnetic shielding enclosures are not suitable for use on GeoSurv II. The inclusion of materials with high magnetic-permeability will alter the natural magnetic field gradient measured by the magnetometer system.

2. Faraday cages are suitable for attenuating high-frequency magnetic fields. The field frequencies that are of principal concern on GeoSurv II are below $10 \mathrm{~Hz}$. 
Higher frequency field components may be filtered using numerical methods.

3. Active shielding systems were reviewed but were determined to be too complicated for the scope of this work. Furthermore it was concluded that static. (time-invariant) magnetic interference would be the focus of the research which negates the necessity of an active attenuation system.

\subsubsection{Low Magnetic Signature Actuators}

1. Piezo-ceramic-based actuation systems such as linear inchworm actuators, morphing wing structures, or hybrid piezo-pneumatic actuators, were identified as the inost promising low magnetic signature actuators. These actuators do not contain ferromagnetic materials, and actuating force is generated using electrical power with a high voltage to current ratio which minimizes electromagnetic fields as well. The commercial availability of such actuators is effectively nonexistant and the effort and research complexity of developing them was beyond the scope of this thesis.

2. Servo actuators are manufactured from a wide range of materials and electric motor types and can easily be integrated into the GeoSurv II aircraft. It was concluded that servo actuators would be adopted for use on the aircraft and that a strategy for attenuating their magnetic interference be developed.

\subsubsection{Magnetic Field Modelling}

Dipole equivalence was selected for modelling sources of magnetic interference on GeoSurv II. Two model frameworks, direct dipole modelling (DDM) and Modified Dipole Ellipse Modelling (MoDEM) were developed and experimentally validated. The following conclusions were reached: 
1. Based on the experiments and analysis presented in Section 5.3, DDM was shown to be unsuitable for modelling sources of interference on GeoSurv II. The parameterization algorithm for estimating the equivalent dipole moment using DDM does not take into consideration the magnetic centroid at which the equivalent dipole acts. During parameterization testing, it was observed that even in the far field region (distance of at least three times the largest dimension of the object) where the field should be dipolar, the eccentricity between the axis of rotation and the magnetic centroid resulted in non-dipolar field characteristics.

2. The MoDEM modelling integrates eccentricity into the model and adds a second dipole vector to reduce model errors. The results presented in Section 5.4 show the MoDEM parameterization strategy should be used for modelling the GeoSurv II actuation system for use in the OFA strategy.

\subsubsection{Optimal Field Attenuation}

A novel strategy called Optimal Field Attenuation was proposed and validated as a method for attenuating, spatially stabilizing, and equalizing (over multiple areas) the magnetic interference produced by sources of magnetic interference. The OFA algorithm was verified experimentally using a set of three ceramic magnets modelled using the MoDEM algorithm. Analysis of OFA led to the following conclusions:

1. The average field over an area of optimization can be reduced using the OFA algorithm. This is beneficial for applications that require robustness to variations in the position of the sensitive components. The ultimate use on GeoSurv II requires further investigation using a more sensitive field measurement system and knowledge of the specific actuators and components to be installed in the aircraft. 
2. A trade-off must be considered between absolute attenuation, optimal field stability, and multi-objective optimal field equality.

3. The study described in Section 6.5 shows that the achievable attenuation decreases proportionally with the size of the optimization area. Conversely the spatial stability of the field is improved with increasing optimization area.

4. When considering multi-objective OFA cases, such as the field gradient system on GeoSurv II, it was shown in Section 6.5.2 that equalizing the magnetic interference at both sensor locations may result in very low differential errors. The size of the differential error was shown to increase proportionally to the size of the optimization areas.

\subsection{Recommendations for Future Work}

The following recommendations may be considered for future work:

1. Improve the MoDEM parameterization strategy to achieve better fit of the vector field components. Most specifically this refers to improving the model precision for the z-component of the magnetic field.

2. Repeat parameterization and OFA tests using a higher resolution magnetometer and improved position and orientation sensing system.

3. Apply OFA to the GeoSurv II aircraft after all actuators have been specified and possible mounting locations established. Determine the optimal configuration of sources and test the algorithm in a real-world application.

4. Further exploring piezo actuation strategies and their associated magnetic signatures. Ultimately, the best strategy for developing a non-magnetic actuating system is to use truly non-magnetic actuators. Based on the research completed 
for this thesis, it is conceivable that a custom piezo actuator will represent the most likely candidate for a very low magnetic signature actuator. 


\section{List of References}

[1] Project. Geosurv II System Requirements Docunnent: Revision E, March 2008. Carleton University Department of Mechanical and Aerospace Engineering.

[2] David Jiles. Introduction to Magnetism and Magnetic Materials. Chapman and Hall, 1991.

[3] Wallace H. Campbell. Introduction to Geomagnetic Fields. Cambridge University Press, 1997.

[4] A.Z. Capri and P.V. Panat. Introduction to Electrodynamics. CRC PRess, 2002.

[5] John David Jackson. Classical Electrodynamics 3rd Ed. John Wiley and Sons, 1999.

[6] John Wilson. Field Geophysics. The Geological Field Guide Series 3rd Ed. John Wiley and Sons, 2002.

[7] Kenichi Yamazaki and Tadashi Kawainoto. Simple Estimation of Equivalent Magnetic Dipole Moment to Characterize ELF Magnetic Fields Generated by Electric Appliences Incorporating Harmonics. IEEE Transactions on Electromagnetic Compatibility, 43(2):240-245, 2001.

[8] Robert G. Olsen and Christopher E. Lyon. Modelling of Extremely Low Frequency Magnetic Field Sources Using Multipole Techniques. IEEE Transactions on Power Delivery, 11(3):1563-1571, 1996.

[9] Luciano E. Zaffanella, Thomas P. Sullivan, and Ivano Visitainer. Magnetic Field Characterization of Electrical Appliances as Point Sources Through in Situ Measurements. IEEE Transactions on Power Delivery, 12(1):443-450, 1997.

[10] Thomas N. Roy: Spacecraft Magnetic Field Modeling. IEEE Transactions on Magnetics, 13(1):914-920, 1977. 
[11] MicroPilot. Specifications - MP2028g. 2008.

[12] Hak-Tae Lee and Ilan M. Kroo. Computational Investigation of Wings witl Miniature Trailing Edge Control Surfaces. In Proceedings of the 2nd AIAA Flow Control Conference, Portland Oregon, 2004.

[13] Justin Manzo, Ephrahim Garcia, Adam Wickenleiser, and Garnett C. Horner. Design of a shape-memory alloy actuated macro-scale morphing aircraft mechanism. Proceedings of SPIE - The International Society for Optical Engineering, $5764(1): 232-240,2004$.

[14] Jacob Joseph Loverich. Development of a New High Specific Power Piezoelectric Actuator. Phd. thesis, Pennsylvania State University, 2004.

[15] Matt Detrick, Seung-Keon Kwak, and Hwan-Sik Yoon. Morphing Flight Control Surface for Advanced Flight Performance. Proceedings of SPIE - The International Society for Optical Engineering. 6173, 2006.

[16] J.C. Bruch Jr, J.M. Sloss amd S. Adali, and I.S. Sadek. Optimal Piezo-actuator Locations/Lengths and Applied Voltage for Shape Control of Beams. Smart Materials and Structures, 9(2):205-211, 2000.

[17] Gareth J. Knowles, Ross Bird, and Ron Barrett. Self-contained High Authority Control for Miniature Flight Systems. Proceedings of SPIE - The International Society for Optical Engineering, 5388:258-265, 2004.

[18] Andrew J. Moskalik and Diann Brei. Force-deflection Behavior of Piezoelectric C-block Actuator Arrays. Smart Materials and Structures, 8(5):531-543, 1999.

[19] David F. Waechter, Shaun Salisbury, Ridha Ben Mrad, S. Eswar Prasad, Richard G. Blacow, and Bin Yan. Complementary Inchworm® Actuator for High-Force High-Precision Applications. Proceedings of SPIE - The International Society for Optical Engineering, 5390(1):13-23, 2001.

[20] Roelof Vos, Ron Barrett, Lars Krakers, and Michel van Tooren. Post-Buckled Precompressed (PBP) Piezoelectric Actuators for UAV Flight Control. Proceedings of SPIE - The International Society for Optical Engineering, 6173, 2006.

[21] Lisa D. Mauck, Jacqueline Menchaca, and Christopher S. Lynch. Piezoelectric Hydraulic Pump Development [for Smart Wing Technology. Proceedings of SPIE - The International Society for Optical Engineering, 3985:729-739, 2000. 
[22] Jayant Sirohi and Inderjit Chopra. Development of a Compact Piezoelectrichydraulic Hybrid Actuator. Proceedings of SPIE - The International Society for Optical Engineering, 4327:401-412, 2001.

[23] Stuart Birch. Technology Update: Don't flap. Aerospace Engineering, page 7, 2006 .

[24] G. Jones, S. Viken, A. Washburn, L. Jenkins, and C. Cagle. An Active Flow Circulation Controlled Flap Concept for General Aviation Aircraft Applications. In Proceedings of the 1st Flow Control Conference, St. Louis, Missouri, 2004.

[25] Steven P. Frith and Norman J. Wood. Investigation of Dual Circulation Control Surfaces for Flight Control. In Proceedings of the 2nd AIAA Flow Control Conference, 2004.

[26] Mark S. Mason and William J. Crowther. Fluidic Thrust Vectoring of Low Observable Aircraft. In CEAS Aerospace Aerodynamic Research Conference, Cambridge, UK, 2002.

[27] Russell Sparks, Stephen Michie, and Ken Gill. Development of an Integrated Circulation Control/Fluidic Thrust Vectoring Flight Test Demonstrator. In 1st International Conference on Innovation and Integration in Aerospace Sciences, Belfast, $U K, 2005$.

[28] Feifei Chen and Shaoxiong Zhou. DC Magnetic Shielding Effectiveness of Soft Composite Sheets. Journal of Magnetism and Magnetic Materials, (239):595$596,2002$.

[29] E. Paperno, H. Koide, and I Sasada. Charts for Estimating the Axial Shielding Factors for Triple-shell Open-ended Cylindrical Shields. IEEE Transactions on Magnetics, 37(4):2881-2883, 2001.

[30] A. Haga, S. Kikuchi, and H. Nasuno. An Elliptical Cylinder Type Shielding Device Using Amorphous Ribbons. IEEE Transactions on Magnetics, 26(5):20112013, 1990.

[31] Craig A. Grimes. EMI Shielding Characteristics of Permalloy Multilayer Thin Films. In Proceedings of IEEE Aerospace Applications Conference, pages 211$221,1994$.

[32] Oriano Bottauscio, Mario Chiampi, and Alessandra Manzin. Numerical Analysis of Magnetic Shielding Efficiency of Multi-layered Screens. IEEE Transactions on Magnetics, 40(2):726-729, 2004. 
[33] C. Buccella, M. Feliziani, and V. Fuina. ELF Magnetic Field Mitigation by Active Shielding. In Proceedings of the 2002 IEEE International Symposium on Industrial Electronics, pages 994-998, 2002.

[34] Sh. M. Shvartsman, R. W. Brown, Y.C. N. Cheng, T. P. Eagan, and J. D. Willig. Supershielding: Trapping of Magnetic Fields. IEEE Transactions on Magnetics, 37(5):3116-3119, 2001.

[35] Matthew Wells, Mojtaba Ahmadi, Paul Straznicky, Malcolm Imray, and Stephen Ferguson. Low Magnetic Signature Actuating System for GeoSurv II UAS. In UVS Canada Conference 2006 - Montebello, Quebec, 2006.

[36] Sheilagh O'Leary. Magnetic Testing of Control Surface Test Bed. Technical Report DR No. 87-01, Carleton University, 2008. 


\section{Appendix A}

\section{Actuator Selection Matrix}

\begin{tabular}{|c|c|c|c|c|c|c|}
\hline & Electro-Magnetic & Hydraulic & Pneumatic & Hybrid Devices & $\begin{array}{l}\text { Solid State } \\
\text { Actuators }\end{array}$ & Flapless Control \\
\hline Examples & $\begin{array}{l}\text { DC Moror: Servo; } \\
\text { Lineat Electric } \\
\text { Actuator; Solenoid; }\end{array}$ & $\begin{array}{l}\text { Hydradic Pistor); } \\
\text { Rotary or I inear }\end{array}$ & $\begin{array}{l}\text { Pnetumatic Piston; } \\
\text { Rosary or Linear }\end{array}$ & $\begin{array}{l}\text { Pion-Hydrstilic } \\
\text { Acutator: lnchworm } \\
\text { Device }\end{array}$ & $\begin{array}{l}\text { Shape Memory } \\
\text { Alloy: Flettrar } \\
\text { active polyner; } \\
\text { Piezo ceranics }\end{array}$ & $\begin{array}{l}\text { Secondary airflow } \\
\text { Coanda effect } \\
\text { fystem }\end{array}$ \\
\hline Power & DC Eertic & 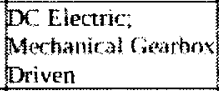 & $\begin{array}{l}\text { DC Electric; } \\
\text { Merhanicat } \\
\text { Cearbox Driven }\end{array}$ & DC Electric & $\begin{array}{l}\text { DC Electric; Hot } \\
\text { Fngine Bleen-air }\end{array}$ & $\begin{array}{l}\text { DC Electric: } \\
\text { Mechanical } \\
\text { Crearbox Deiven }\end{array}$ \\
\hline Drive Principle & $\begin{array}{l}\text { Force/torque is a } \\
\text { function of magnetic } \\
\text { field strengtis (applied } \\
\text { current) }\end{array}$ & $\begin{array}{l}\text { Force is applicel } \\
\text { hrough pressurized } \\
\text { fluid applied over } \\
\text { piston area }\end{array}$ & $\begin{array}{l}\text { Eorce is applied } \\
\text { chrough } \\
\text { compressed gas } \\
\text { gpplied oner piston } \\
\text { areat }\end{array}$ & $\begin{array}{l}\text { Applied efectic } \\
\text { potential produces a } \\
\text { dimensional change } \\
\text { in the PZT }\end{array}$ & $\begin{array}{l}\text { Dimensimat change } \\
\text { Broduced through: } \\
\text { femperature (SMA) } \\
\text { or eletric potential } \\
\text { (FAP. PTT) }\end{array}$ & $\begin{array}{l}\text { Arfiow over the } \\
\text { wing is redinected } \\
\text { by merins of a } \\
\text { secondary flow of } \\
\text { hir expelled } \\
\text { hrongh the trailing } \\
\text { edge }\end{array}$ \\
\hline Key Components & $\begin{array}{l}\text { Amplifer; Cables: } \\
\text { Magnetic Shielding }\end{array}$ & $\begin{array}{l}\text { Purnp: Reservoir; } \\
\text { Working flaid; } \\
\text { Sensors; } \\
\text { Accumulator: } \\
\text { Fiters; Valves; } \\
\text { Fluid Lines }\end{array}$ & $\begin{array}{l}\text { Compressor; } \\
\text { Pressure Vessel; } \\
\text { Sensors; fitters; } \\
\text { Valves; Flow l.ines }\end{array}$ & $\begin{array}{l}\text { DC-DC Converter; } \\
\text { Amplifier; Sensors; } \\
\text { Cables TBD }\end{array}$ & $\begin{array}{l}\text { DE-DC Comverter; } \\
\text { Sensors; Cables; } \\
\text { TED }\end{array}$ & $\begin{array}{l}\text { Control Algorithim; } \\
\text { Wing Redesign; } \\
\text { wir Compressor: } \\
\text { Valves; JBD }\end{array}$ \\
\hline Integratability & High & Possible & Possible & TBD. & IBD. & NA \\
\hline $\begin{array}{l}\text { Magnetic Field } \\
\text { Effects }\end{array}$ & $\begin{array}{l}\text { High (resedrching } \\
\text { mitigation }\end{array}$ & Manageable & Manageable & $\begin{array}{l}\text { BD. Theoretically } \\
\text { low }\end{array}$ & $\begin{array}{l}\text { LBD. Theoretically } \\
\text { Low }\end{array}$ & $\begin{array}{l}\text { Te. Potentially } \\
\text { Very Low }\end{array}$ \\
\hline $\begin{array}{c}\text { Cost (materials + } \\
\text { labour) }\end{array}$ & low + low & high + medium & Low + medium & high + mediam & TBD + high & high + high \\
\hline Stroke & Adequate & Adequate & Actequate & 180 & Not Adeguate & Nid \\
\hline Bandwidth & Good & Good & Low & $1 \mathrm{BD}$ & $1 \mathrm{BD}$. & NA \\
\hline Controllability & High & Good & poor & LBD. & Highly Complex & NA \\
\hline
\end{tabular}


Appendix B

\section{Data Acquisition Scripts}




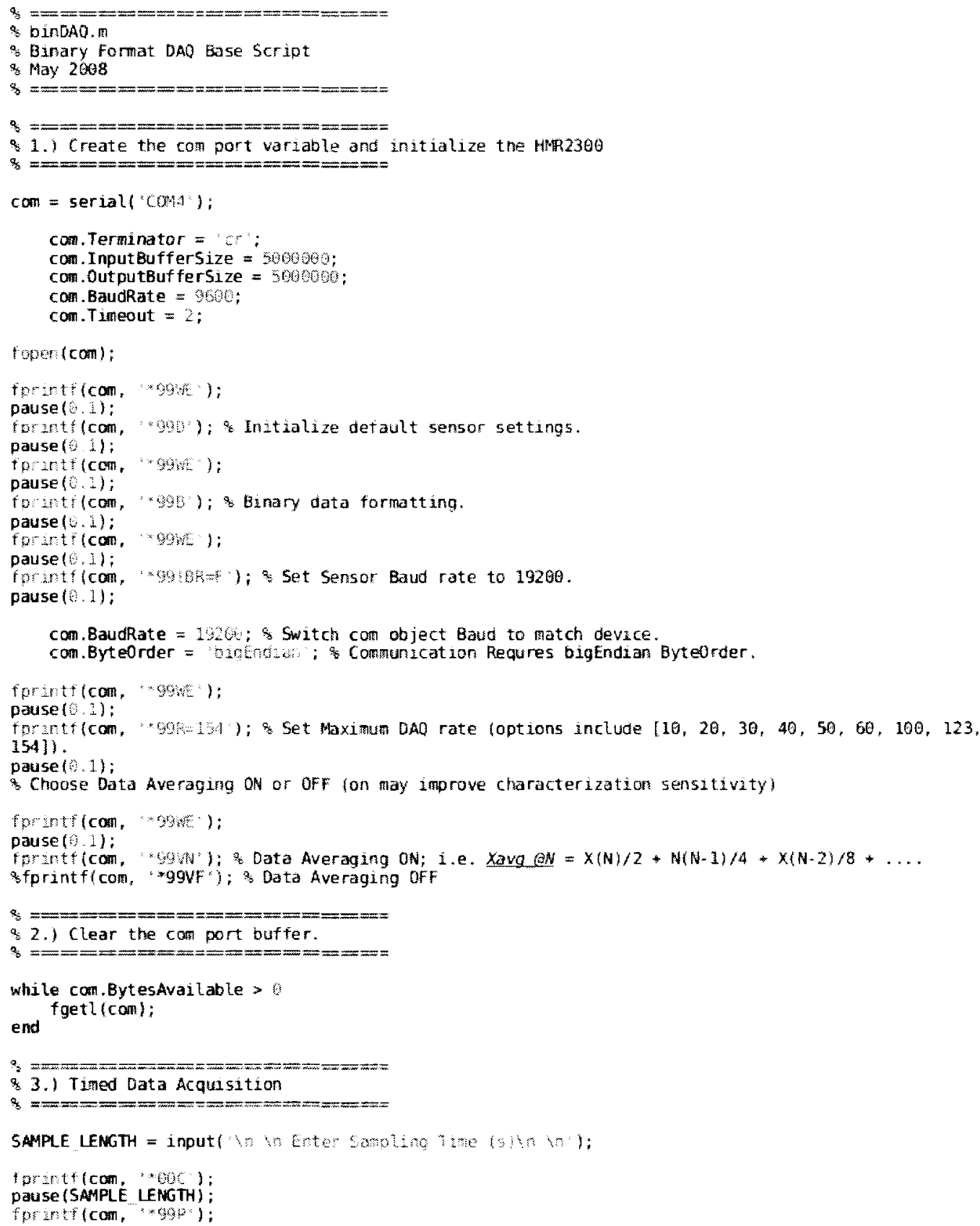

SAMPLES TAKEN = Momó(con. BytesAvailablef7) - i: so Binary dato is reao as a 7 byte string. 
$B=2 \operatorname{ergs}(2,5$ AMPLES TAKEN);

for $k=1:$ SAMPLES TAKEN

$B(:, k)=$ fread (com, 3 , 'short's:

$B(:, k)=(100 * B(i, k)\} / 1 S: \&$ Conversion to $n t$

discard = freatio (com, $x$, "shat" $)$;

end

$B \operatorname{mag}(k)=\operatorname{norm}(B(:, k))$; sok $\angle A G R O N=$ s field Magnitude

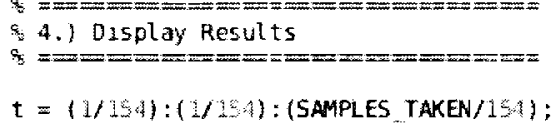

figure( ()

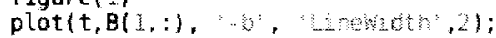

xlabel $(2)(x)$

ylabel (Fux benty $\mathrm{b}$ (n)

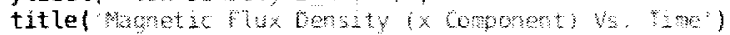

figure( 2$)$

plot $(t, B(2, s),-b, \operatorname{mensth}, 2)$;

xlabel( tame (s) )

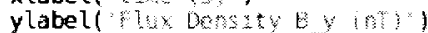

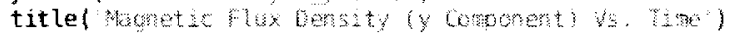

figure( 3$)$

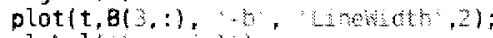

xlabel( tasecis:

ylabel( whe Gensity $\mathrm{E} z$ in)

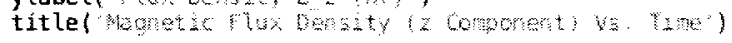

figure( 1$)$

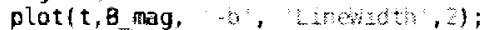

xlabel ( $\mathrm{f}, \mathrm{mat}(\mathrm{s})$ )

ylabel(

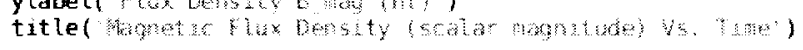

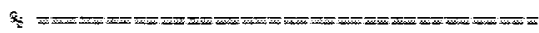

4.) Terminate Serial Connection and Clean variables

o : $=$

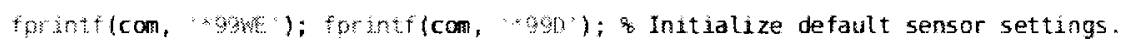
fscanf (com); fscanf (com) :

folose(com):

$B z=B(3,:) ;$

$\mathrm{By}=\mathrm{B}(2,:)$

$\mathrm{Bx}=\mathrm{B}(\mathrm{h}, \mathrm{i})$

characterization. means $=[$ mean $(B x) ; \operatorname{mean}(B y) ;$ mean $(B z)]$

characterization. stdevs $=[s \operatorname{td}(B x) ; s \operatorname{td}(B y) ; s t d(B z)]$ :

clear $k$ B ans com 


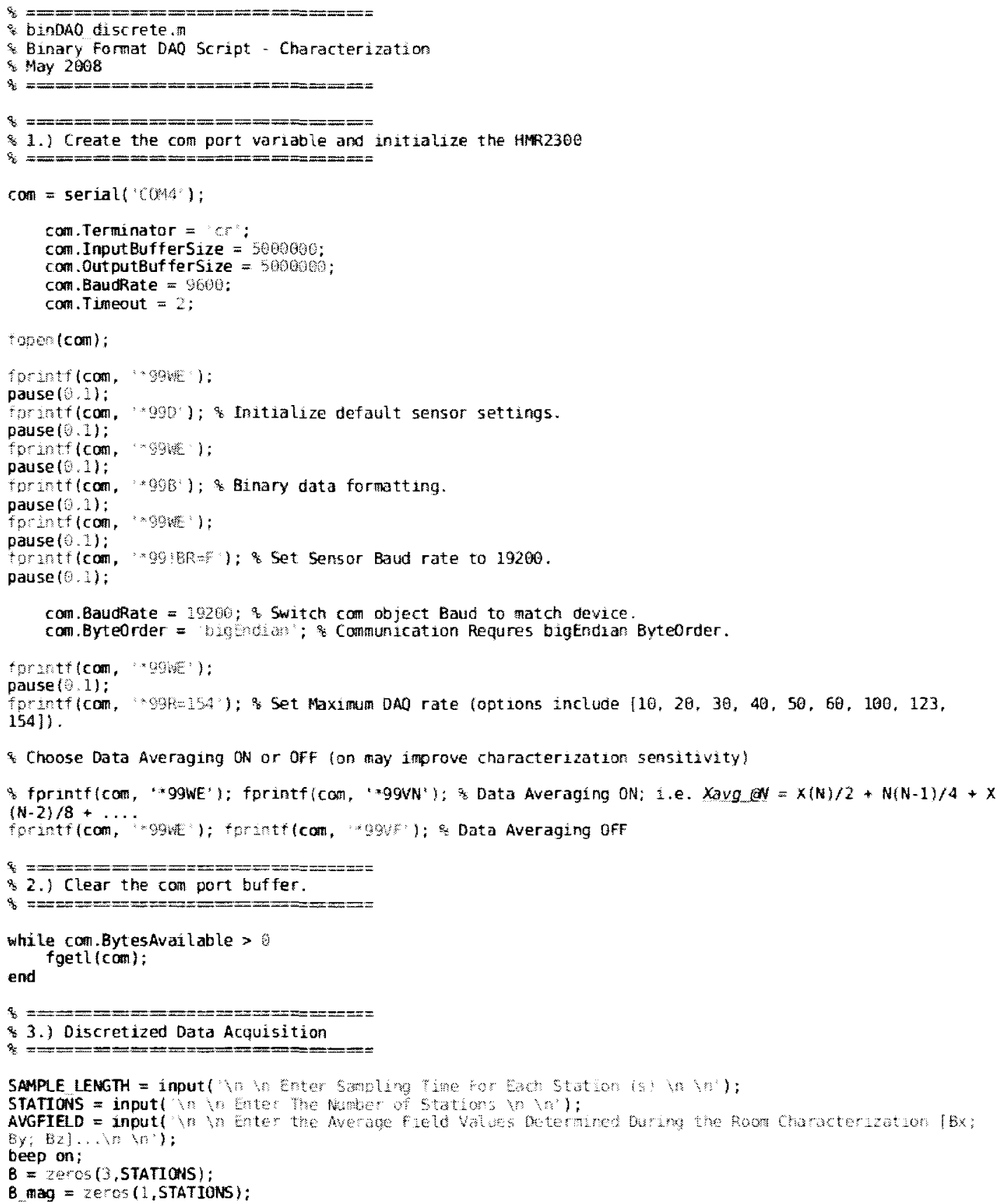




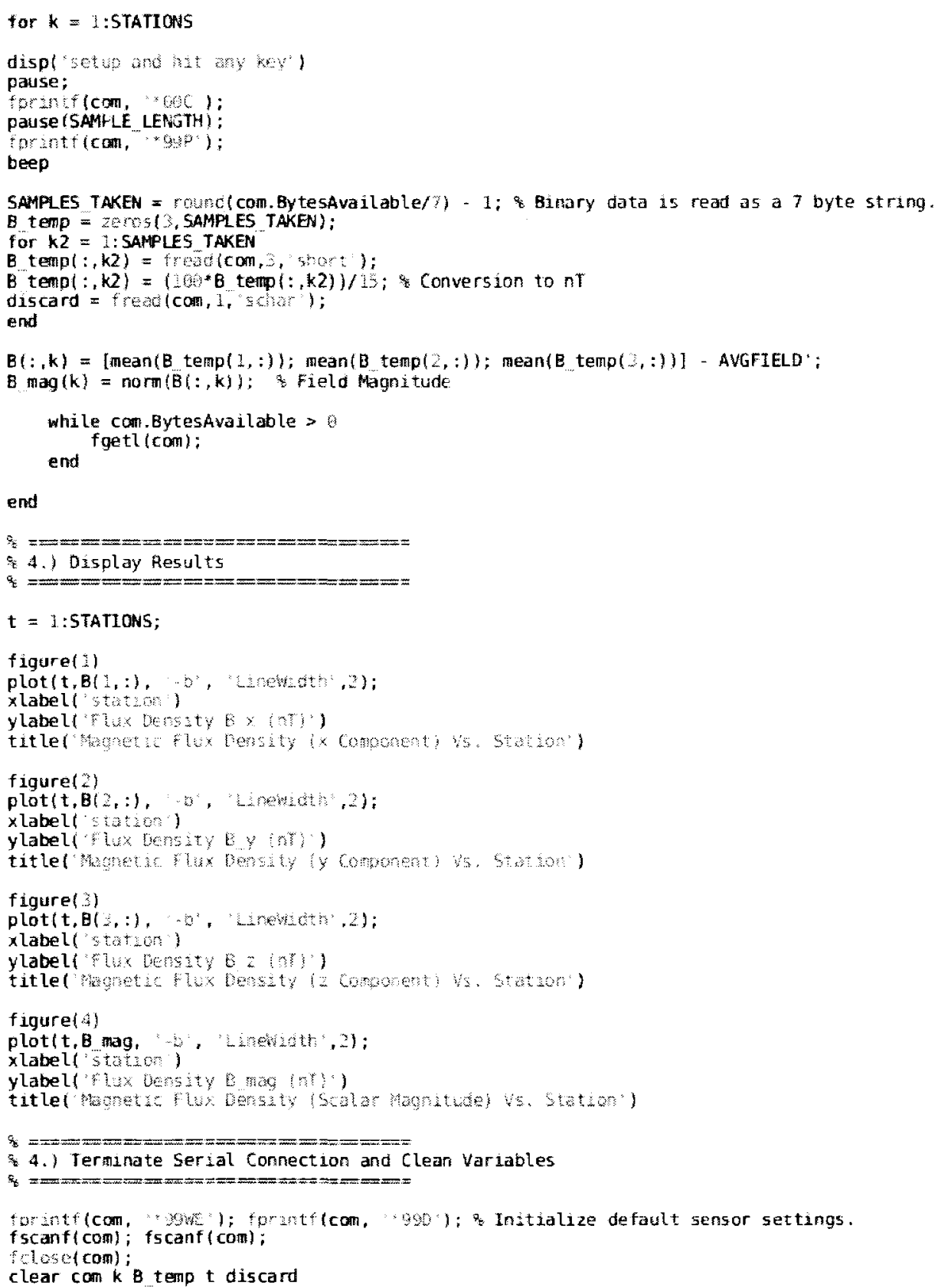


Appendix C

DDM and MoDEM Parameterization

Scripts 
* Direct Dipole Solvirg Algorithm

\& May 2008

\& Rev3

\& XYZ field date (corrected for background error) must be calculated prior

s to runing this script.

$\$$

I IAPUT THE FOLLOWING PARAMETERS

$r=[0.3 ; 0]$; 2 position of the sensor with respect to the source; in meters $v=r$ inorm $(r)$;

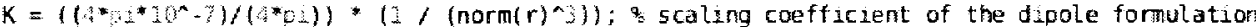

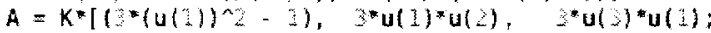

3*u(1)*u(2), (3*u(2) $\left.)^{2}-3\right), 3 u(3) * u(3)$

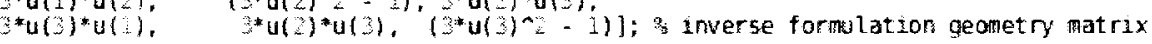

$a=0.0 .2018: 2 * 5+0.2818 ;$ s increnents of rotation angle (radians)

$b=\operatorname{logt} n(a)$

2. PROCESSING

M estimatel $=$ zerse $(s, b)$

M estimate2 = zerou ( b)

for $k=1: b$

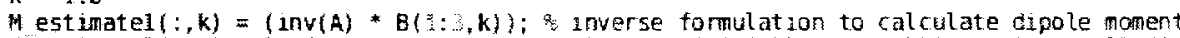

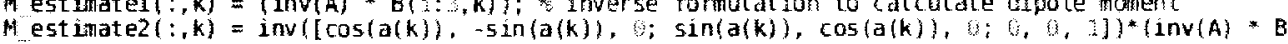

$(i: s, k)$ ): irverse formulation to calculate aipole moment

end

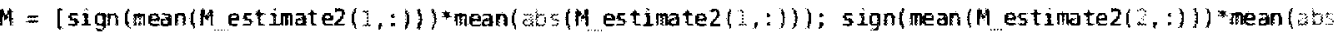

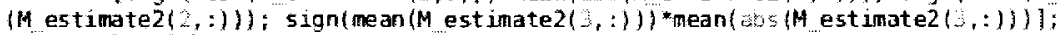

for $k=1: b$

B estimate $(: k)=A^{*}([\cos (a(k)),-\sin (a(k)), 0 ; \sin (a(k)), \cos (a(k)), 0 ;, 0,1]=$

$\operatorname{cro}(:, k)=B(:, k)-B$ estinate $(:, k)$ :

Bmag $(k)=\operatorname{nom}(B(:, k))$

Bmag estimate $(k)=\operatorname{norm}(B$ estimate $(:, k))$

end

mager romormal ize $(k)=200 *($ mag $(k)$-Bmag estimate $(k)) / B$ mag $(k)$

\& CLEAN VARIABLES

rot $=6: 15: 360 ;$

clear $k$ b $r$ ans $K$ a $A$ 


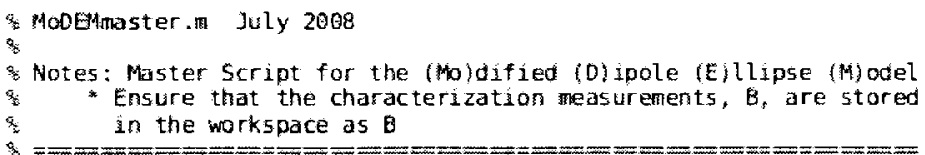

(1) Initialize Test variables...

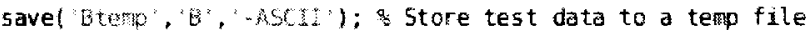

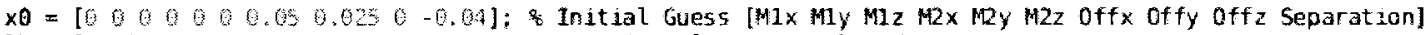

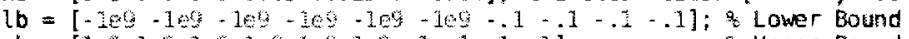

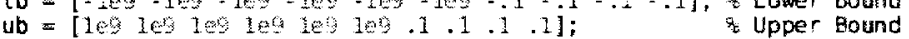

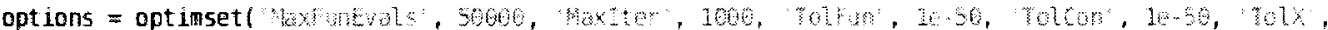
$16-50$, plot ins, coptimplotfvali;

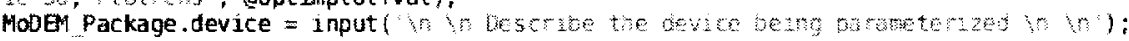

(2) call foincon (run the solver)

[optx, fval, EXITFLAG, OUTPUT, LAHBDA,GRAD, HESSIAN] = fmincon(etoDEMobjective, $x \theta,[],[],[]$,

[], lb, ub, (Mooeqconstraint, options):

(3) Package the Results

MoDeM Package. offset $=$ optx $(7 ; 0)$;

MoDEM Package.ellipseoffset $=$ opt $\times(10)$

MoDEM Package. $M 1=\operatorname{opt} \times(1: 3)$;

MoDEM Package $M 2=\operatorname{apt} x(A: 5)$

[MoDEM Package. Bxyz measure, MoDEq Package.Bxyz model] = MoDEMtest (optx):

MODEM Package.Bxyz Error = MoDEM Package. Bxyz measure - MoOEM Package. Bxyz model:

for $k=1: 1$ exot (HoDEM Package. Bxyz measure)

MoDel Package. Emag model $(k)=$ nom (NODEM Package. Bxyz model $(: k))$; $k$-AGROAs

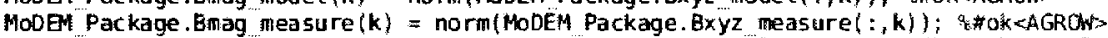

MoDEM Package. Bmag PercentError $(k)=10$ * (MODEM Package. Bmag measure $(k)$. MoDEM Package. Bmag model (k) )/MODEM Package. Bnag measure(k);

end

Mober Package.output $=$ OUTPUT;

clear ub $x \theta$ lo $k$ options optx EXITFLAG LANEDA HESSIAN GRAD fval OUTPUT

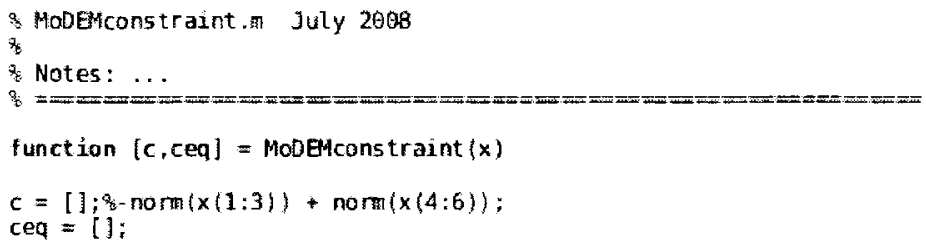




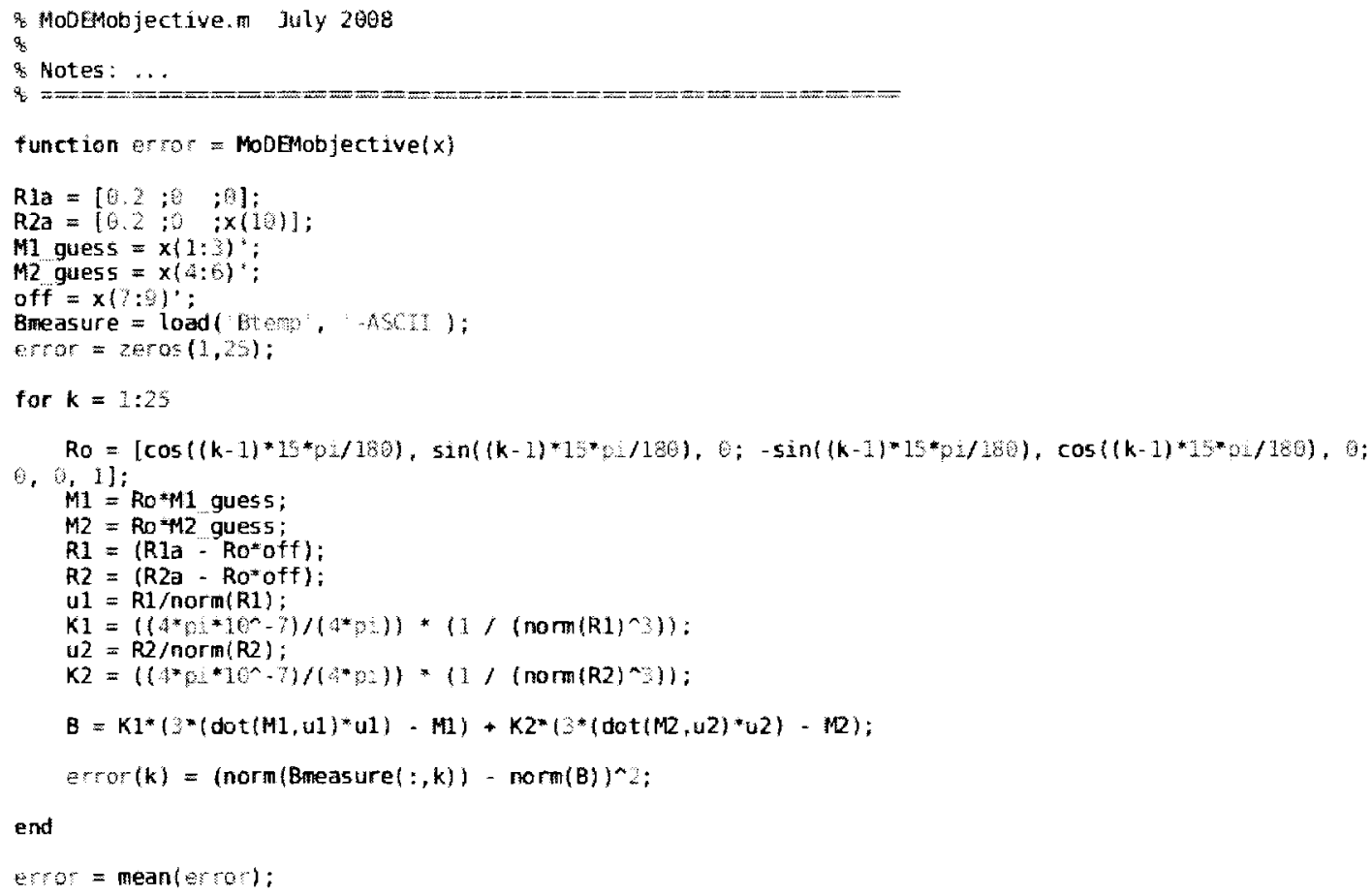


Appendix D

OFA Scripts 


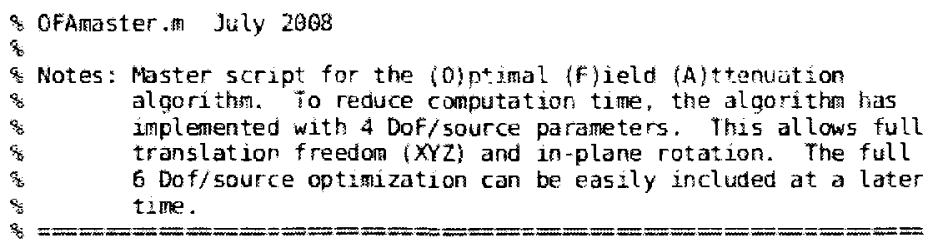

S. (1.) Initialize Variables...

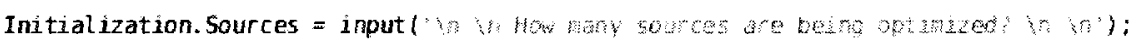

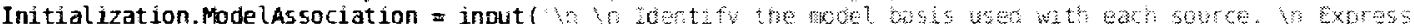

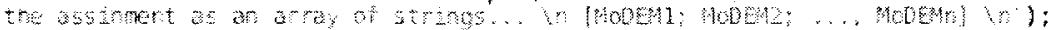

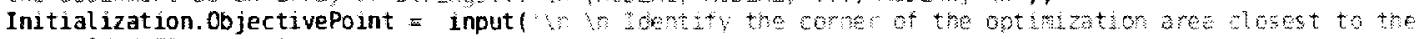
aren $\{x, y, z\}$ in $\{:$

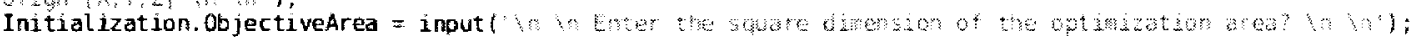

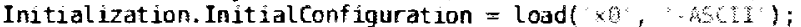

Initial ization. UpperBound = load ("w, 'ASCl:').

Initialization. LowerBound $=$ load $(16 ;-\sec 2)$;

NOTE: The HoDEM models should be saved as workspace variables in the same

\& directory as this script. start, upperbound, and lowerbound should be

i saved as text files, format as a $\left[X_{1}, Y_{1}, 21\right.$, Rot $1 ; \ldots ; X_{n}, Y_{n}, 2 \mathrm{n}$,

Rotri...

$x \theta=[1 ;$

$\mathrm{Ub}=[1$;

for $k=1$ :Initial ization. Sources

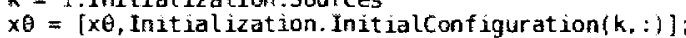

lb $=\left[\right.$ [b. Initialization. Lowe $\left.{ }^{\text {Bound }}(\mathrm{k},:)\right]$;

end

$u b=$ lub, Initialization. UpperBound $(k,:)]$ :

savel mitaliation"):

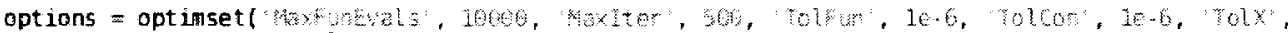
leb, 'plutros', (optimplotfval):

s.

(3.) Rur the fmincon solver...

[Initialfield.Bx, Initialfield.By, Initialfield.Bz, Initialfield.Bmag] = OFASim $(x \theta)$;

[optx, fval, EXITFLAG, OUTPUT, LAMBOA, GRAD, HESSIAN] = fmincon(@OFAobjective, $x \theta,[1,1], 1], 1], \mathrm{b}, \mathrm{b}$,

[1, options):

[Finalfield.Bx, Finalfield.By, Finalfield.Bz, Finalfield.Bmag] = OFAsim( optx):

optimum. Configuration $=$ zeros $($ Initialization. Sources, 4$)$;

Opt imum. Meanfield $=\mathrm{fval}$;

for $k=1$ :Initialization. Sources

end

Opt imum.Configuration $(k,:)=\operatorname{opt} \times(1+(k-1) * 4: 4+(k-1) * 4)$;

(4.) Process Data and Clear Unnecessary Variables...

for $x=1: 106$

for $y=1: 100$

Del ta.Bx(x,y) $=($ Initialfield. $B x(x, y)) /($ Finalf leld. Bx $(x, y)))$

Del ta.By $(x, y)=$ (Initialfield.By $(x, y))$ /((Finalfield. By $(x, y)))$;

Delta.Bz $(x, y)=$ (Initialfield. $B z(x, y)) /$ /(finalfield. $B z(x, y))):$

end

Delta.Bmag $(x, y)=($ Initialfield.Bmag( $x, y)) /($ fFinalfield.Bmag $(x, y)))$;

end

clear EXITFLAG GRAG LANBOA HESSIAN $k$ lo ub $x$ y $\times 6$ meanfield fval $S$ optx 


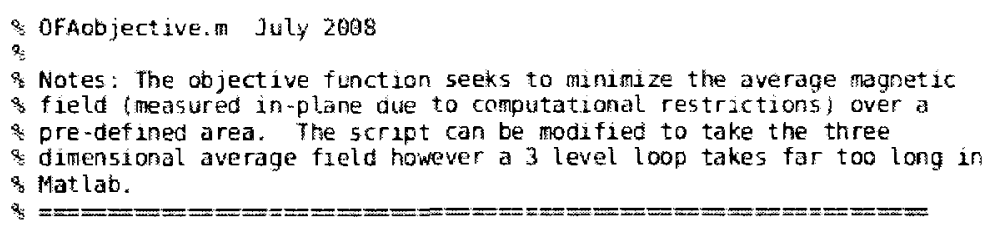

function [meanfield] = OfAobjective( $x$ )

s. 1.) Initialize

load (mithatzation): : Lad the initialize Data from the OfAnaster Script

$s=$ Initialization objectivearea 1 :

$B x=$ zeros (Initialization. 0bjectiveArea/s):

By $=$ zeros (Initialization. ObjectiveArea/5):

$\mathrm{Bz}=$ zeros(Initialization. ObjectiveArea/S);

Bmag = zeros(Initialization. DojectiveArea/S)

Tx = zeros(Initialization. Ob jectivearea/5): * Temporary variables

$T y=$ zarses (Initialization.0bjectiveArea/S): Tegorary Variables

$\mathrm{Tz}=2 \cos$ (Initialization. ObjectiveArea/S); so Temporany Variables

state = zeros(Initialization. Sources. $A$ );

for $k=1:$ Initialization. Sources

end

state $(k,:)=x(1+(k-1) \times 4: 4+(k-1) * 4)$;

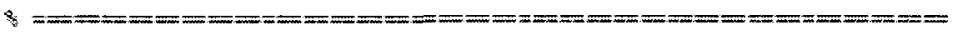

3.) Solve

for $k=1$ initialization.Sources

load(Initialization. Made LAssoriation( $k,:))$;

for $y=2:$ Initialization.objectiveArea/S

for $x \bar{~} 1$ initialization. objectivearea/s

R1ini = Initialization .0bjectivePoint $+((x-1) * S:(y-1) * 5 ; 0]-$ state $(k, 1: 3)^{2}$;

R2ini = Initialization .0bjectivePoint + $[(x-1) * 5:(y-1) * 5 ; 0]-5 t a t e(k, 1: 3)^{\prime}$

[0; : MoDEM Package. ell ipseof fset]:

Rot $=[\cos (\operatorname{state}(k, 4)),-\sin (\operatorname{state}(k, 4)), 0 ; \sin (\operatorname{state}(k, 4)), \cos (5 \operatorname{tate}(k, 4)), 0 ; 0,0,1] ;$

$M 1=$ Rot MoDEH Package. M1';

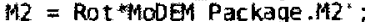

$R 1=\left(\right.$ Rlini - Rot MODEM Package . of $f$ set $\left.^{2}\right):$

$R 2=(\text { Rzini }- \text { Rot'MoDEM Package off set" })^{*}$;

$\mathrm{u} 1=\mathrm{R} 1 / \mathrm{nom}(\mathrm{R} 1)$

formulation

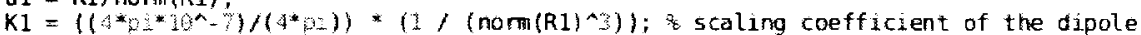

$u 2=R 2 /$ nom $(R 2)$

formulation

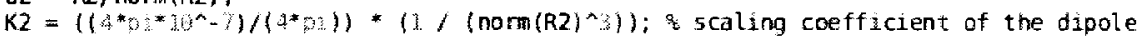

$B=K 1 *(2 *(\operatorname{dot}(M 1, u 1) * u 1)-M 1)+K 2 *(\geqslant *(\operatorname{dot}(M 2, u 2) * u 2)-M 2) ;$

$T x(x, y)=B(1) ;$

$T y(x, y)=B(z) ;$
$T z(x, y)=8(3):$

$\gamma(x, y)=B(3):$

end

$B x=B x+T x ;$

$B y=B y+T Y ;$

$B z=B z+T z ;$

for $y=1:$ Initialization.objectiveArea/s

for $x=$ :Initialization.objectiveArea/s

Bmag $(x, y)=\operatorname{norm}([B x(x, y) ; B y(x, y) ; B z(x, y)])$ :

end

end

meanfield = mean (mean (Bmag) ):

clear $x$ y Tx Ty $T z$ state Rot R1 R1ini R2 R2ini M1 M2 MOOEM Package $K 1 K_{2} k$ u1 u2 a $S$ 


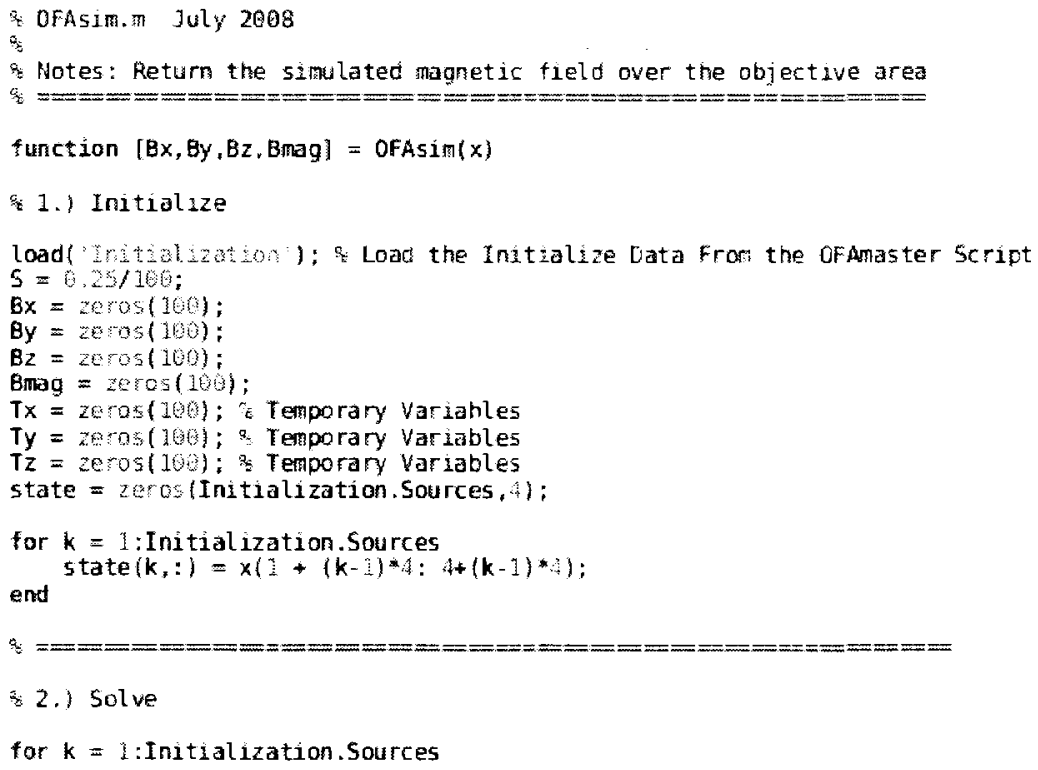

